\title{
Álgebras de Lie Semi-simples
}

\author{
Leonardo Gomes Oliveira
}




\title{
Álgebras de Lie Semi-simples
}

\author{
Leonardo Gomes Oliveira
}

Orientador: Prof. Dr. Daniel Levcovitz

Dissertação apresentada ao Instituto de Ciências Matemáticas e de Computação - ICMC-USP, como parte dos requisitos para obtenção do título de Mestre em Matemática.

USP - São Carlos

Janeiro/2009 


\section{Resumo}

A dissertação tem como tema as álgebras de Lie. Especificamente álgebras de Lie semi-simples e suas propriedades . Para encontramos essas propriedades estudamos os conceitos básicos da teoria das álgebras de Lie e suas representações. Então fizemos a classificação dessas álgebras por diagramas de Dynkin explicitando quais os possíveis diagramas que são associados a uma álgebra de Lie semi-simples. Por fim, demonstramos vários resultados concernentes a essa classificação, dentre esses, o principal resultado demonstrado foi: os diagramas de Dynkin são um invariante completo das álgebras de Lie semi-simples. 



\begin{abstract}
The dissertation has the theme Lie algebras. Specifically semi-simple Lie algebras and its properties. To find these properties we studied the basic concepts of the theory of Lie algebras and their representations. Then we did the classification by Dynkin diagrams of these algebras and explaining the possible diagrams that are associated with a semi-simple Lie algebra. Finally, we demonstrate several results related to this classification, among these, the main result demonstrated was: the Dynkin diagrams are a complete invariant of semi-simple Lie algebras.
\end{abstract}





\section{Sumário}

Introdução

1 Preliminares $\quad 3$

1.1 Definições . . . . . . . . . . . . . . . . . . . . . . 3

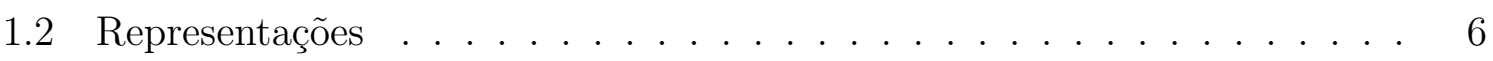

1.2.1 Decomposições de Representações . . . . . . . . . . . . . . . . . . 7

1.3 Séries de Composição . . . . . . . . . . . . . . . . . . . . . . . . . . . . . . . . .

1.4 Álgebras simples e Álgebras semi-simples . . . . . . . . . . . . . . . . . . . 10

1.5 Representações de Álgebras nilpotentes e solúveis . . . . . . . . . . . . . . 10

1.6 Critérios de Cartan . . . . . . . . . . . . . . . . . . . . . . . 12

1.7 Subálgebras de Cartan . . . . . . . . . . . . . . . . . . . 13

2 Representação Adjunta de Álgebras semi-simples $\quad 15$

2.1 Representações de sl(2) . . . . . . . . . . . . . . . . . . . 15

2.2 Subálgebras de Cartan . . . . . . . . . . . . . . . . . . . 18

2.3 Fórmula de Killing . . . . . . . . . . . . . . . . . . . . . . . . . . . . 23

2.4 Sistema simples de raízes . . . . . . . . . . . . . . . . . . . . 27

2.5 Matrizes de Cartan . . . . . . . . . . . . . . . . . . . . . 31

2.6 Diagramas de Dynkin . . . . . . . . . . . . . . . . . . . 35

2.7 Exemplo de Álgebra Semi-Simples . . . . . . . . . . . . . . . . . . 36

3 Diagramas de Dynkin 39

3.1 Classificação dos diagramas . . . . . . . . . . . . . . . . . . . . . . 39

3.2 Realizações dos diagramas . . . . . . . . . . . . . . . . . . . . 48

4 Álgebras Semi-simples $\quad 51$

4.1 Álgebras Isomorfas e seus Diagramas de Dynkin . . . . . . . . . . . . . . 51

4.2 Realizações das àlgebras clássicas . . . . . . . . . . . . . . . . . 61

$\begin{array}{ll}\text { Bibliografia } & 71\end{array}$ 


\section{Introdução}

Esta dissertação tem como objetivo estudar resultados concernentes à teoria das álgebras de Lie. Mais especificamente às álgebras de Lie semi-simples e suas representações.

O único pré-requisito para o entendimento do trabalho é a álgebra linear. Vale citar que para estudar os conceitos mais avançados da teoria foi preciso o estudo de toda teoria relacionada a essas álgebras de Lie e em particular sua relação com os espaços vetoriais. Por exemplo: a decomposição de Jordan de matrizes, as formas lineares e bilineares, o espaço das transformações lineares, os autovalores das transformações e seus autovetores correspondentes, auto-espaços generalizados e bases de espaços vetoriais.

O desenvolvimento da dissertação se dará de modo usual. Após enunciarmos os conceitos preliminares, desenvolveremos a teoria de modo claro e sucinto. Os detalhes dos cálculos serão feitos sempre que necessário. As conclusões serão apresentadas enfatizando sua importância para os próximos tópicos.

No primeiro capítulo, das preliminares, serão enunciados vários conceitos básicos das álgebras de Lie de dimensão finita. Definições básicas de: morfismos, ideais, subálgebras, representações, séries de composição, álgebras solúveis, álgebras nilpotentes e finalmente álgebras simples e semi-simples. Também enunciaremos resultados relacionados a Forma de Cartan-Killing, subálgebras de Cartan e decomposição da álgebra pela representação adjunta da subálgebra de Cartan.

A partir do segundo capítulo iremos nos concentrar apenas no estudo das álgebras semi-simples e suas representações. O primeiro passo é ver como se comportam as representações irredutíveis da álgebra $s l(2)$. Também demonstraremos alguns resultados das subálgebras de Cartan de uma álgebra semi-simples, sua representação adjunta e os pesos relacionados a essa representação. Então falaremos sobre pesos (raízes), Fórmula de Killing, sistemas simples de raízes e seus diagramas de Dynkin associados. Cada peso irá corresponder a uma subálgebra isomorfa a $s l(2)$, assim sempre poderemos ver a álgebra $s l(2)$ incluída na álgebra semi-simples. Por fim, vamos mostrar como classificar (representar) uma álgebra semi-simples por um diagrama de Dynkin (grafo).

No terceiro capítulo mostraremos alguns resultados da teoria de diagramas de Dynkin. Encontraremos os possíveis diagramas que representam as álgebras e suas propriedades. $\mathrm{Na}$ última seção, iremos expor as bases de espaços vetoriais sobre o corpo dos racionais que realizam os diagramas.

No último capítulo serão abordados os resultados mais importantes que relacionam 
as álgebras de Lie semi-simples com os diagramas de Dynkin. O primeiro resultado mostra que os diagramas são invariantes em relação a subálgebras de Cartan distintas de uma mesma álgebra. Outro fato importante é que os diagramas de duas álgebras isomorfas coincidem. Finalmente, o principal resultado que será demonstrado é: os diagramas de Dynkin são um invariante completo das álgebras semi-simples, ou seja, se duas álgebras têm o mesmo diagrama então é possível construir um isomorfismo entre essas álgebras.

Em seguida a esse teorema principal faremos a realização de alguns dos possíveis diagramas encontrados no terceiro capítulo, a partir de álgebras semi-simples. Ou seja, iremos mostrar que dado um diagrama, realmente existem uma álgebra semi-simples, sua subálgebra de Cartan, seu sistema simples de raízes correspondentes que, visto como uma base de um espaço vetorial racional, têm as mesmas propriedades (números de Killing) das bases encontradas no capítulo três. Será feito a construção das álgebras clássicas, que são basicamente subálgebras da álgebra das matrizes.

Esses são os resultados apresentados em nossa dissertação. Ela foi baseada essencialmente no livro "Álgebras de Lie", de Luiz A. B. San Martin. Vale ressaltar que o conteúdo presente na dissertação é de bastante utilidade para quem está se iniciando nesta área, pois expõe as definições, proposições e teoremas imprescindíveis a quem deseja compreender um texto simples ou um artigo mais complexo, que tenha como tema álgebras de Lie e suas representações. 


\section{Capítulo 1}

\section{Preliminares}

Este primeiro capítulo serve para expor as definições e conceitos básicos da teoria de Álgebras de Lie. Vamos enunciar alguns resultados sem demonstração, pois se trata apenas de apresentar o material teórico e fixar a notação que vão ser necessários para os capítulos subsequentes.

\subsection{Definições}

Definição 1.1.1. Uma Álgebra de Lie consiste de um espaço vetorial $\mathfrak{g}$ munido de uma operação (colchete de Lie) entre os vetores

$$
[,]: \mathfrak{g} \times \mathfrak{g} \longrightarrow \mathfrak{g}
$$

que tem as seguintes propriedades:

1. [, ] é bilinear,

2. [, ] é anti-simétrica, ou seja, $[X, X]=0$ para todo $X \in \mathfrak{g}$. (Equivale a dizermos que $[X, Y]=-[Y, X]$ para todo $X, Y \in \mathfrak{g})$,

3. [, ] satisfaz a identidade de Jacobi, isto é, para todo $X, Y, Z \in \mathfrak{g}$,

$$
[X,[Y, Z]]+[Z,[X, Y]]+[Y,[Z, X]]=0 .
$$

A identidade de Jacobi pode ser escrita de outras maneiras, como

$$
[X,[Y, Z]]=[[X, Y], Z]+[Y,[X, Z]]
$$

$\mathrm{Ou}$

$$
[[X, Y], Z]=[[X, Z], Y]+[X,[Y, Z]] .
$$

Sabemos que um álgebra em geral é espaço vetorial $\mathfrak{g}$ munida de uma aplicação bilinear de $\mathfrak{g} \times \mathfrak{g}$ em $\mathfrak{g}$. Uma álgebra de Lie tem a anti-simetria e a identidade de Jacobi como características específicas. Assim todos os conceitos básicos de espaço vetorial se estendem naturalmente para álgebras de Lie, como por exemplo um subespaço, que no caso de uma álgebra é chamado subálgebra. 
Definição 1.1.2. Seja $\mathfrak{g}$ uma álgebra de Lie. Uma subálgebra $\mathfrak{h}$ de $\mathfrak{g}$ é um subespaço vetorial que é fechado pelo colchete, isto é, $[X, Y] \in \mathfrak{h}$ se $X, Y \in \mathfrak{h}$.

Exemplo 1.1.3. Um exemplo de álgebra de Lie que será mencionado em toda a dissertação é a álgebra das transformações lineares de um espaço vetorial de dimensão $n$ sobre um corpo $\mathbb{K}$, que será denotado por $g l(n, \mathbb{K})$ ou simplesmente $g l(V)$. Essa álgebra é a mesma que o espaço das matrizes $n \times n$ com coeficientes em $\mathbb{K}$ e o colchete é dado por

$$
[X, Y]=X Y-Y X
$$

Aqui $X Y$ é a operação de composição entre transformações lineares que é o mesmo que a multiplicação de matrizes.

Definição 1.1.4. Sejam $\mathfrak{g}_{1}$ e $\mathfrak{g}_{2}$ duas álgebras de Lie. Uma transformação linear $\varphi$ : $\mathfrak{g}_{1} \rightarrow \mathfrak{g}_{2}$ é um

- homomorfismo se $\varphi[X, Y]=[\varphi X, \varphi Y]$, para todo $X, Y \in \mathfrak{g}_{1}$;

- isomorfismo se for um homomorfimo inversível;

- automorfismo se é um isomorfismo e $\mathfrak{g}_{1}=\mathfrak{g}_{2}$.

Definição 1.1.5. Duas álgebras $\mathfrak{g}_{1}$ e $\mathfrak{g}_{2}$ são ditas isomorfas se existe um isomorfismo $\varphi: \mathfrak{g}_{1} \rightarrow \mathfrak{g}_{2}$.

Uma forma de verificar que duas álgebras de Lie de dimensão finita são isomorfas é através dos colchetes de Lie entre seus elementos das bases. Seja $\mathfrak{g}$ uma álgebra de Lie e $\left\{X_{1}, \ldots, X_{n}\right\}$ uma base de $\mathfrak{g}$. Tome $X_{i}$ e $X_{j}$ dois elementos quaisquer da base. Podemos escrever o colchete entre esses dois elementos como combinação linear da base

$$
\left[X_{i}, X_{j}\right]=\sum_{k=1}^{n} c_{i j}^{k} X_{k} .
$$

Os coeficientes $c_{i j}^{k}$ que aparecem na expressão acima são denominados constantes de extrutura da álgebra em relação a base fixada. Portanto duas álgebras de Lie são isomorfas se suas constantes de estrutura são iguais. As constantes de estrutura satisfazem as seguintes igualdades: para toda terna $i, j, k$ temos,

$$
\begin{gathered}
c_{i j}^{k}=-c_{j i}^{k}, \\
\sum_{l, m}\left(c_{i j}^{l} c_{l k}^{m}+c_{j k}^{l} c_{l i}^{m}+c_{k i}^{l} c_{l j}^{m}\right)=0 .
\end{gathered}
$$

A primeira igualdade deve-se à anti-simetria do colchete e a segunda à identidade de Jacobi. Reciprocamente, dadas constantes que satisfazem essas duas igualdades, elas são constantes de estrutura de uma álgebra de Lie. De fato, tomando uma base $\left\{X_{1}, \ldots, X_{n}\right\}$ de um espaço vetorial, definindo $\left[X_{i}, X_{j}\right]=c_{i j}^{k} X_{k}$ e estendendo por bilinearidade, obtémse um álgebra de Lie cujas constantes estruturais são $c_{i j}^{k}$. 
Definição 1.1.6. Um subespaço $\mathfrak{h} \subset \mathfrak{g}$ é um ideal se para todo $Y \in \mathfrak{h}$ e todo $X \in \mathfrak{g}$ temos $[X, Y] \in \mathfrak{h}$, ou seja,

$$
[\mathfrak{g}, \mathfrak{h}] \subset \mathfrak{h}
$$

É claro que todo ideal é um subálgebra mas não vale a recíproca.

$\operatorname{Seja~} \varphi: \mathfrak{g}_{1} \rightarrow \mathfrak{g}_{2}$ um homomorfismo, então

$$
\begin{gathered}
\text { ker } \varphi \text { é um ideal de } \mathfrak{g}_{1}, \\
i m \varphi \text { é uma subálgebra de } \mathfrak{g}_{2} \text {. }
\end{gathered}
$$

Definição 1.1.7. Seja $\mathfrak{g}$ um álgebra de Lie e $\mathfrak{h} \subset \mathfrak{g}$ um ideal. No espaço vetorial quociente $\mathfrak{g} / \mathfrak{h}$ defina

$$
[\bar{X}, \bar{Y}]=\overline{[X, Y]}
$$

onde $\bar{X}$ denota a classe $X+\mathfrak{h}$. Definindo assim o colchete, o espaço $\mathfrak{g} / \mathfrak{h}$ é uma álgebra de Lie.

Aqui também, como em todas as teorias clássicas da álgebra, vale o teorema dos isomorfismos:

Teorema 1.1.8. 1. Seja $\varphi: \mathfrak{g}_{1} \rightarrow \mathfrak{g}_{2}$ um homomorfismo de álgebras de Lie. Então,

$$
\mathfrak{g} / \operatorname{ker} \varphi \approx i m \varphi
$$

2. Sejam $\mathfrak{g}$ uma álgebra de Lie e $\mathfrak{h}_{1}, \mathfrak{h}_{2} \subset \mathfrak{g}$ ideais. Então,

$$
\left(\mathfrak{h}_{1}+\mathfrak{h}_{2}\right) / \mathfrak{h}_{1} \approx \mathfrak{h}_{2} / \mathfrak{h}_{1} \cap \mathfrak{h}_{2}
$$

A demonstração desse teorema é a mesma que para grupos, anéis ou espaços vetoriais.

Definição 1.1.9. Sejam $\mathfrak{g}_{1}, \ldots, \mathfrak{g}_{n}$ álgebras de Lie e

$$
\mathfrak{g}=\mathfrak{g}_{1} \oplus \ldots \oplus \mathfrak{g}_{n}
$$

sua soma direta como espaços vetoriais. Com o colchete

$$
[X, Y]=\left(\left[X_{1}, Y_{1}\right], \ldots,\left[X_{n}, Y_{n}\right]\right)
$$

onde $X=\left(X_{1}, \ldots, X_{n}\right)$ e $Y=\left(Y_{1}, \ldots, Y_{n}\right)$, define-se uma estrutura de álgebra de Lie no espaço vetorial produto $\mathfrak{g}$. 


\subsection{Representações}

Um dos conceitos que mais estudaremos nesse trabalho é o de representação de uma álgebra de Lie em um espaço vetorial. Essa é uma das ferramentas mais eficazes para encontrar a estrutura da álgebra, como por exemplo a existência de ideais e subálgebras.

Definição 1.2.1. Seja $V$ um espaço vetorial e $g l(V)$ a álgebra das transformações lineares de $V$ (que pode ter dimensão finita ou não). Seja $\mathfrak{g}$ uma álgebra de Lie sobre o mesmo corpo de escalares que $V$. Uma representação de $\mathfrak{g}$ em $V$ é um homomorfismo de álgebras de Lie

$$
\rho: \mathfrak{g} \longrightarrow g l(V)
$$

O espaço vetorial $V$ é denominado espaço de representação e sua dimensão é chamada dimensão da representação. Uma representação $\rho$ é dita fiel se ela é injetora, ou seja, $\operatorname{ker} \rho=\{0\}$.

Quando ocorre da representação ser fiel, pelo teorema dos isomorfismos $\mathfrak{g}$ é isomorfa a $i m \rho$, que é uma subálgebra de $g l(V)$.

Outra maneira de introduzirmos o conceito de representação é vendo a álgebra das transformações lineares como um módulo sobre $\mathfrak{g}$.

Definição 1.2.2. Um módulo sobre um álgebra de Lie $\mathfrak{g}$ é um espaço vetorial juntamente com uma operação de multiplicação $\mathfrak{g} \times V \rightarrow V$, denotada por $(X, v) \mapsto X v$, que satisfaz:

1. $(X+Y) v=X v+Y v$,

2. $X(u+v)=X u+X v$,

3. $a X v=X(a v)$,

4. $[X, Y] v=X Y v+Y X v$,

para todo $X, Y \in \mathfrak{g}, u, v \in V$ e $a \in \mathbb{K}$.

Um módulo V sempre define uma representação

$$
\begin{array}{rlll}
\rho: \quad \mathfrak{g} \longrightarrow & g l(V) & \\
X \longrightarrow \rho(X): \quad & \quad \rightarrow \quad V \\
& & v \mapsto \rho(X) v=X v .
\end{array}
$$

Analogamente, pelo mesmo diagrama acima, uma representação define um módulo. Basta tomar $X v=\rho(X) v$. Portanto, os conceitos de módulo e representação são equivalentes.

Dentre as representações de álgebras de Lie, há uma mais importante. Considere, para cada elemento $X \in \mathfrak{g}$, a transformação linear

$$
\operatorname{ad}(X): \mathfrak{g} \longrightarrow \mathfrak{g}
$$

definida por $\operatorname{ad}(X) Y=[X, Y]$. 
Proposição 1.2.3. A aplicação de $\mathfrak{g}$ em $\operatorname{gl}(\mathfrak{g})$ ( $\mathfrak{g}$ visto como espaço vetorial)

$$
a d: X \in \mathfrak{g} \longmapsto a d(X) \in g l(\mathfrak{g})
$$

é uma representação de $\mathfrak{g}$ em $\mathfrak{g}$.

Essa representação é denominada representação adjunta. O núcleo da representação adjunta é denominado centro de $\mathfrak{g}$ e é denotado por $\mathfrak{z}(\mathfrak{g})$ :

$$
\mathfrak{z}(\mathfrak{g})=\{X \in \mathfrak{g}: \operatorname{ad}(X) Y=[X, Y]=0, \forall Y \in \mathfrak{g}\} .
$$

A representação adjunta será a mais estudada neste trabalho e é dela que vem os principais resultados que encontraremos nos capítulos posteriores.

Definição 1.2.4. Sejam $\rho_{1}$ e $\rho_{2}$ duas representações de $\mathfrak{g}$ em $V_{1}$ e $V_{2}$, respectivamente. Elas são ditas equivalentes se existe um isomorfimo de espaços vetoriais $P: V_{1} \rightarrow V_{2}$ tal que

$$
\rho_{2}(X) \circ P=P \circ \rho_{1}(X)
$$

para qualquer $X \in \mathfrak{g}$.

Dada uma representação $\rho$ de $\mathfrak{g}$ em $V$, pode-se tomar a presentação $\rho^{*}$ de $\mathfrak{g}$ em $V^{*}$. Essa representação é dada pela fórmula

$$
\rho^{*}(\lambda)(X)=-\lambda \circ \rho(X), \quad \lambda \in V^{*}, X \in \mathfrak{g} .
$$

Essa representação $\rho^{*}$ é denominada dual da representação $\rho$. Em particular denotamos $a d^{*}$ a representação dual da adjunta, que é denominada representação co-adjunta.

Definição 1.2.5. Seja $\rho$ uma representação de $\mathfrak{g}$ em $V$ e suponha que $W$ é um subespaço de $V$ tal que $\rho(X) W \subset W$, para todo $X \in \mathfrak{g}$. Esse subsespaço $W$ é chamado subespaço invariante, e a restrição $\rho_{\mid W}$ define uma representação

$$
\begin{aligned}
\rho_{\mid W}: & \mathfrak{g} \longrightarrow g l(W) \\
& X \longrightarrow \rho(X)
\end{aligned}
$$

Observação 1.2.6. Um subespaço $\mathfrak{h} \subset \mathfrak{g}$ é invariante pela representação adjunta se e somente se $\mathfrak{h}$ é um ideal de $\mathfrak{g}$.

\subsubsection{Decomposições de Representações}

Nesta seção nos preocuparemos com o problema de decompor o espaço vetorial $V$, espaço da representação $\rho$ da álgebra de Lie $\mathfrak{g}$. Queremos saber quando podemos escrever o espaço $V$ como soma direta de subespaços invariantes. 
Definição 1.2.7. Uma representação $\rho$ de $\mathfrak{g}$ em $V$ é dita irredutível se os únicos subespaços invariantes por $\rho$ são os subespaços triviais $\{0\}$ e $V$. A representação é dita completamente redutivel se $V$ se decompõe como

$$
V=V_{1} \oplus \ldots \oplus V_{n}
$$

onde cada $V_{i}$ é invariante e tal que a restrição de $\rho$ a $V_{i}$ é irredutível.

A decomposição de $V$ em componentes irredutíveis não é única. Pela definição vemos que uma representação irredutível é sempre completamente redutível. A seguir enunciaremos uma proposição que é bastante útil quando queremos saber se uma representação é completamente redutível.

Proposição 1.2.8. Seja $\rho$ uma representação de dimensão finita de $\mathfrak{g}$ em $V$. Então $\rho$ é completamente redutivel se, e somente se, todo subespaço invariante admite um complementar invariante. Isto é, para todo $W$ subespaço invariante, existe um subespaço invariante $W_{1}$ tal que

$$
V=W \oplus W_{1}
$$

Para concluirmos essa seção iremos definir um tipo especial de aplicação linear entre álgebras que será importante para o estudo da representação adjunta.

Definição 1.2.9. Uma aplicação linear $D: \mathfrak{g} \rightarrow \mathfrak{g}$ é uma derivação da álgebra de Lie $\mathfrak{g}$ se satifaz

$$
D[X, Y]=[D X, Y]+[X, D Y] \text { para todo } X, Y \in \mathfrak{g} .
$$

Essa é a regra de Leibniz de derivada de um produto.

Observe que a aplicação $a d(X)$ é uma derivação, pois a igualdade requerida é exatamente a identidade de Jacobi. As derivações desse tipo são denominadas derivações internas.

\subsection{Séries de Composição}

Seja $\mathfrak{g}$ uma álgebra de Lie então denotamos por $[\mathfrak{g}, \mathfrak{g}]$ o conjunto

$$
\{[X, Y]: X \in \mathfrak{g}, Y \in \mathfrak{g}\} .
$$

Definição 1.3.1. Definimos, por indução, os subespaços:

$$
\begin{gathered}
\mathfrak{g}^{(0)}=\mathfrak{g} \\
\mathfrak{g}^{\prime}=[\mathfrak{g}, \mathfrak{g}] \\
\vdots \\
\mathfrak{g}^{(k)}=\left[\mathfrak{g}^{(k-1)}, \mathfrak{g}^{(k-1)}\right] .
\end{gathered}
$$

Essa série é conhecida por série derivada de $\mathfrak{g}$. 
Prova-se facilmente que esses subespaços são na verdade ideais de $\mathfrak{g}$.

Definição 1.3.2. Uma álgebra de Lie $\mathfrak{g}$ é dita solúvel se existe um $k_{0} \geq 1$, tal que,

$$
\mathfrak{g}^{\left(k_{0}\right)}=\{0\} .
$$

Proposição 1.3.3. 1. Se $\mathfrak{g}$ é solúvel $e \mathfrak{h} \subset \mathfrak{g}$ é subálgebra então $\mathfrak{h}$ é solúvel.

2. Se $\mathfrak{g}$ é solúvel e $\mathfrak{h} \subset \mathfrak{g}$ é um ideal, então $\mathfrak{g} / \mathfrak{h}$ é solúvel.

Proposição 1.3.4. Seja $\overline{\mathbb{K}}$ é uma extensão de $\mathbb{K}$. $\mathfrak{g}$ é solúvel se, e somente se, $\mathfrak{g}_{\overline{\mathbb{K}}}$ é solúvel.

Definição 1.3.5. Definimos agora, outra sequência de subespaços:

$$
\begin{gathered}
\mathfrak{g}^{1}=\mathfrak{g} \\
\mathfrak{g}^{2}=[\mathfrak{g}, \mathfrak{g}]=\mathfrak{g}^{\prime} \\
\vdots \\
\mathfrak{g}^{k}=\left[\mathfrak{g}, \mathfrak{g}^{k-1}\right] .
\end{gathered}
$$

Essa série é chamada série central descendente da álgebra $\mathfrak{g}$.

Proposição 1.3.6. A série derivada decresce mais rápido que a série central descendente:

$$
\mathfrak{g}^{(k)} \subset \mathfrak{g}^{k+1}
$$

Definição 1.3.7. Uma álgebra de Lie é nilpotente se existe um $k_{0} \geq 1$ tal que,

$$
\mathfrak{g}^{k_{0}}=\{0\}
$$

Observe que toda álgebra nilpotente é em particular solúvel.

Proposição 1.3.8. 1. Se $\mathfrak{g}$ é nilpotente $e \mathfrak{h} \subset \mathfrak{g}$ é subálgebra então $\mathfrak{h}$ é nilpotente.

2. Se $\mathfrak{g}$ é nilpotente e $\mathfrak{h} \subset \mathfrak{g}$ é um ideal, então $\mathfrak{g} / \mathfrak{h}$ é nilpotente.

Proposição 1.3.9. Seja $\overline{\mathbb{K}}$ é uma extensão de $\mathbb{K}$. g é nilpotente se, e somente se, $\mathfrak{g}_{\overline{\mathbb{K}}}$ é nilpotente.

Proposição 1.3.10. Seja $\mathfrak{g}$ uma álgebra de Lie de dimensão finita. Então, existe em $\mathfrak{g}$ um único ideal solúvel $\mathfrak{r}$ que contém todos os ideais solúveis de $\mathfrak{g}$.

Definição 1.3.11. O ideal $\mathfrak{r}$ da proposição anterior é chamado de radical solúvel (ou simplesmente radical) de $\mathfrak{g}$. Para o radical de $\mathfrak{g}$ será utilizada a notação $\mathfrak{r}(\mathfrak{g})$.

Proposição 1.3.12. Seja $\mathfrak{g}$ uma álgebra de Lie de dimensão finita. Então, existe em $\mathfrak{g}$ um único ideal $\mathfrak{n} \mathfrak{n}$ que contém todos os ideais nilpotentes de $\mathfrak{g}$.

Definição 1.3.13. O ideal $\mathfrak{n} \mathfrak{n}$ da proposição anterior é chamado de radical nilpotente (ou nil-radical) de $\mathfrak{g}$. Para o radical de $\mathfrak{g}$ será utilizada a notação $\mathfrak{r} \mathfrak{n}(\mathfrak{g})$. 


\section{4 Álgebras simples e Álgebras semi-simples}

A partir desta seção denotaremos por 0 o ideal $\{0\}$ quando for claro que estamos falando de conjuntos e não do elemento 0 .

Definição 1.4.1. Uma álgebra de Lie $\mathfrak{g}$ é semi-simples se

$$
\mathfrak{r}(\mathfrak{g})=0
$$

(isto é, gãa contém ideais solúveis além de 0).

Definição 1.4.2. Uma álgebra $\mathfrak{g}$ é simples se

1. Os únicos ideais de $\mathfrak{g}$ são 0 e $\mathfrak{g}$.

2. $\operatorname{dim} \mathfrak{g} \neq 1$.

A propriedade 2., da álgebra simples, de que tenha dimensão diferente de um, é para que exista compatibilidade entre os conceitos de álgebras simples e semi-simples.

\subsection{Representações de Álgebras nilpotentes e solúveis}

Nesta seção serão enunciados resultados importantes das representações de álgebras nilpotentes e solúveis. O principal teorema desta teoria é o teorema de Engel, que nos diz que, para uma álgebra de Lie de transformações lineares cujos elementos são nilpotentes, é possível encontrar uma base em que as matrizes desses elementos são todas triangulares superiores com zeros na diagonal principal.

Definição 1.5.1. Seja $\mathfrak{g}$ um álgebra de Lie. Uma representação $\rho$ de $\mathfrak{g}$ no espaço vetorial $V$ é uma representação nilpotente se dado $X \in \mathfrak{g}$, existe um inteiro positivo $k$ (dependente de $X$ ) tal que $\rho(X)^{k}=0$.

Teorema 1.5.2. Seja $V$ um espaço vetorial de dimensão finita e $\mathfrak{g} \subset \mathrm{gl}(V)$ uma subálgebra tal que todo $X \in \mathfrak{g}$ é nilpotente. Então existem subespaços

$$
0=V_{0} \subset V_{1} \subset \ldots \subset V_{n-1} \subset V_{n}=V
$$

tal que $X V_{i} \subset V_{i-1}, i=1, \ldots, m$. Esse subespaços podem ser definidos indutivamente por

$$
\begin{gathered}
V_{0}=0 \\
V_{i}=\left\{v \in V: X v \in V_{i-1} \forall X \in \mathfrak{g}\right\} .
\end{gathered}
$$

Em particular, estendendo sucessivamente as bases dos subespaços $V_{i}$, chega-se em uma base de $V$ tal que a matriz de $X$ em relação a essa base é triangular superior com zeros na diagonal para todo $X \in \mathfrak{g}$.

Teorema 1.5.3. Seja $\mathfrak{g}$ um álgebra de Lie de dimensão finita e suponha para todo $X \in \mathfrak{g}, \operatorname{ad}(X)$ é nilpotente. Então, $\mathfrak{g}$ é nilpotente. 
Teorema 1.5.4. Suponha que o corpo de escalares é algebricamente fechado e tome um representação de $\mathfrak{g}$ em $V$, com $\operatorname{dim} V<\infty$ e $\mathfrak{g}$ nilpotente. Então, existem funcinais lineares $\lambda_{1}, \ldots, \lambda_{s}$ tal que se

$$
V_{\lambda_{i}}=\left\{v \in V: \forall X \in \mathfrak{g}, \exists n \geq 1,\left(\rho(X)-\lambda_{i}(X)\right)^{n} v=0\right\},
$$

então $V_{\lambda_{i}}$ é $\mathfrak{g}$-invariante, $i=1, \ldots, s . E$

$$
V=V_{\lambda_{1}} \oplus \ldots \oplus V_{\lambda_{s}}
$$

Definição 1.5.5. Seja $\mathfrak{g}$ uma álgebra de Lie e $\rho$ uma representação de $\mathfrak{g}$ em $V$. Um peso de $\rho$ é um funcional linear $\lambda: \mathfrak{g} \rightarrow \mathbb{K}$ tal que o subespaço $V_{\lambda}$ de $V$ definido por

$$
V_{\lambda}=\left\{v \in V: \forall X \in \mathfrak{g}, \exists n \geq 1,\left(\rho(X)-\lambda_{i}(X)\right)^{n} v=0\right\},
$$

satisfaz $V_{\lambda} \neq 0$. O subespaço $V_{\lambda}$ é chamado de subespaço de pesos associado a $\lambda$. A dimensão de $V_{\lambda}$ é chamada de multiplicidade de $\lambda$.

Os pesos de uma representação são por definição os autovalores dos elementos da álgebra. O teorema 1.5.4 garante que representações de dimensão finita, de um álgebra nilpotente, sobre um corpo algebricamente fechado admitem pesos. Agora enunciaremos o teorema de Engel.

Teorema 1.5.6. Suponha que o corpo é algebricamente fechado e seja $\rho$ uma representação da álgebra nilpotente $\mathfrak{g}$ sobre o espaço de dimensão finita $V$. Então, existe uma base de $V$ tal que nessa base $\rho$ se escreve como

$$
\rho(X)=\left(\begin{array}{ccc}
\rho_{1}(X) & & \\
& \ddots & \\
& & \rho_{s}(X)
\end{array}\right) X \in \mathfrak{g}
$$

com os blocos diagonais $\rho_{i}(X)$ da forma

$$
\rho_{i}(X)=\left(\begin{array}{ccc}
\lambda_{i}(X) & & * \\
& \ddots & \\
0 & & \lambda_{i}(X)
\end{array}\right) \quad X \in \mathfrak{g} .
$$

Onde $\lambda_{i}$ é um peso da representação.

Esse resultado é o mais importante no contexto de álgebras nilpotente. Observe que essa decomposição mostra que um representação de uma álgebra nilpotente é irredutível se e somente se ela tem dimensão um.

Agora iremos enunciar um resultado semelhante só que para álgebras solúveis. É o teorema de Lie: 
Teorema 1.5.7. Sejam $V$ um espaço vetorial de dimensão finita sobre um corpo algebricamente fechado e $\mathfrak{g} \subset \operatorname{gl}(V)$ uma álgebra solúvel. Então, existe uma base $\beta=$ $\left\{v_{1}, \ldots, v_{n}\right\}$ de $V$ e funcionais lineares $\lambda_{1}, \ldots, \lambda_{n}: \mathfrak{g} \rightarrow \mathbb{K}$ tal que, em relação a $\beta, X \in \mathfrak{g}$ se escreve como

$$
X=\left(\begin{array}{ccc}
\lambda_{1}(X) & & * \\
& \ddots & \\
0 & & \lambda_{n}(X)
\end{array}\right) .
$$

Proposição 1.5.8. Seja $\mathfrak{g}$ um álgebra de Lie de dimensão finita. Então, $\mathfrak{g}$ é solúvel se e somente se a álgebra derivada $\mathfrak{g}^{\prime}$ é nilpotente.

\subsection{Critérios de Cartan}

Nesta seção enunciaremos resultados que relacionam a forma de Cartan-Killing com a estrutura da álgebra, ou seja, se a álgebra é semi-simples ou solúvel. Também enuciaremos resultados que serão úteis mais a frente no desenvolvimento do trabalho.

Definição 1.6.1. Tome a representação adjunta de $\mathfrak{g}$. Definimos a forma de CartanKilling em $\mathfrak{g}$ como

$$
\langle X, Y\rangle=\operatorname{tr}(\operatorname{ad}(X) \operatorname{ad}(Y)), X, Y \in \mathfrak{g} .
$$

Proposição 1.6.2. Seja $D: \mathfrak{g} \rightarrow \mathfrak{g}$ uma derivação da álgebra de Lie de dimensão finita sobre um corpo algebricamente fechado. Tome a decomposição primária

$$
\mathfrak{g}=\mathfrak{g}_{\lambda_{1}} \oplus \ldots \oplus \mathfrak{g}_{\lambda_{m}}
$$

onde

$$
\mathfrak{g}_{\lambda_{i}}=\left\{X \in \mathfrak{g}:\left(D-\lambda_{i}\right)^{n} X=0 \text { para algum } n \geq 1\right\}
$$

é o auto-espaço generalizado associado ao autovalor $\lambda_{i}$. Então,

$$
\left[\mathfrak{g}_{\lambda_{i}}, \mathfrak{g}_{\lambda_{j}}\right] \subset \mathfrak{g}_{\lambda_{i}+\lambda_{j}}
$$

$\left(\mathfrak{g}_{\lambda_{i}+\lambda_{j}}=0\right.$ se $\lambda_{i}+\lambda_{j}$ não é autovalor de $\left.D\right)$.

Teorema 1.6.3. Seja $\mathfrak{g}$ uma álgebra de Lie de dimensão finita, tem-se que $\mathfrak{g}$ é solúvel se, e somente se,

$$
\langle X, Y\rangle=0
$$

para todo $X \in \mathfrak{g}^{\prime}$ e $Y \in \mathfrak{g}$.

Teorema 1.6.4. A forma de Cartan-Killing é não-degenerada em $\mathfrak{g}$ se, e somente se, $\mathfrak{g}$ é semi-simples.

Proposição 1.6.5. Seja $\overline{\mathbb{K}}$ uma extensão de $\mathbb{K}$. Então $\mathfrak{g}$ é semi-simples se, e somente se, $\mathfrak{g}_{\overline{\mathbb{K}}}$ é semi-simples. 
Teorema 1.6.6. Seja $\mathfrak{g}$ uma álgebra semi-simples de dimensão finita. Então, $\mathfrak{g}$ se decompõe em soma direta

$$
\mathfrak{g}=\mathfrak{g}_{1} \oplus \ldots \oplus \mathfrak{g}_{s}
$$

com $\mathfrak{g}_{i}, i=1, . ., s$, ideais simples. Nessa decomposição $\left[\mathfrak{g}_{i}, \mathfrak{g}_{j}\right]=0$ se $i \neq j$. Além do mais,

1. o ortogonal $\mathfrak{g}_{i}^{\perp}$ de uma componente simples, em relação a forma de Cartan-Killing, é a soma das demais componentes,

2. os ideais de $\mathfrak{g}$ são somas de algumas dessas componentes e

3. a decomposição é única (a menos de permutação dos índices).

Proposição 1.6.7. Suponha que $\mathfrak{g}$ seja semi-simples. Então, toda derivação é uma derivação interna, ou seja, se $D$ é uma derivação então existe $X \in \mathfrak{g}$ tal que $D=\operatorname{ad}(X)$.

Corolário 1.6.8. Suponha que $\mathfrak{g}$ seja semi-simples e seja $X \in \mathfrak{g}$. Então, $X$ se decompõe de maneira única em

$$
X=X_{S}+X_{N}
$$

com $X_{S}, X_{N} \in \mathfrak{g}$ tais que ad $\left(X_{S}\right)$ é semi-simples, ad $\left(X_{N}\right)$ é nilpotente e

$$
\left[X_{S}, X_{N}\right]=\left[X, X_{S}\right]=\left[X, X_{N}\right]=0
$$

\subsection{Subálgebras de Cartan}

Nesta seção enunciaremos um conceito muito importante para o estudo das álgebras semi-simples, que é a subálgebra de Cartan.

Definição 1.7.1. O normalizador de uma subálgebra $\mathfrak{h} \subset \mathfrak{g}$ é definido como

$$
\mathfrak{n}(\mathfrak{h})=\{X \in \mathfrak{g}: \operatorname{ad}(X) \mathfrak{h}=[X, \mathfrak{h}] \subset \mathfrak{h}\}
$$

Definição 1.7.2. Seja $\mathfrak{g}$ uma álgebra de Lie. Uma subálgebra de Cartan de $\mathfrak{g}$ é uma subálgebra $\mathfrak{h} \subset \mathfrak{g}$ que satisfaz:

1. h é nilpotente.

2. O normalizador de $\mathfrak{h}$ em $\mathfrak{g}$ coincide com $\mathfrak{h}$.

Será importante o estudo da representação adjunta de uma subálgebra de Cartan $\mathfrak{h}$ em $\mathfrak{g}$. Os pesos não-nulos dessa representação serão denominados raízes de $\mathfrak{h}$.

Agora precisamos defininir um tipo específico de elemento de uma álgebra de Lie $\mathfrak{g}$. Para isso, tome a representação adjunta de $\mathfrak{g}$. Tome o polinômio característico de $\operatorname{ad}(X)$

$$
p_{X}(\lambda)=\lambda^{n}+p_{n-1}(X) \lambda^{n-1}+\ldots+p_{1}(X) \lambda+p_{0}(X)
$$

onde $n=\operatorname{dim} \mathfrak{g}$ e cada $p_{i}(\cdot)$ é um polinômio de grau $n-i$ em $X$. 
Definição 1.7.3. O posto de uma álgebra de Lie de dimensão finita é o menor índice $i$ em que $p_{i}$ não é identicamente nulo, onde $p_{i}$ denota, como acima, os coeficientes dos polinômios característicos de cada $a d(X)$. Um elemento $X \in \mathfrak{g}$ é dito regular se $p_{i}(X) \neq 0$ onde $i$ é o posto de $\mathfrak{g}$.

Teorema 1.7.4. Seja $X \in \mathfrak{g}$ e denote por $\mathfrak{g}_{0}(X)$ o auto-espaço generalizado associado ao autovalor nulo na decomposição primária

$$
\mathfrak{g}=\mathfrak{g}_{0}(X) \oplus \mathfrak{g}_{\lambda_{1}} \oplus \ldots \oplus \mathfrak{g}_{\lambda_{k}}
$$

de $\operatorname{ad}(X)$ com $\lambda_{1}, \ldots, \lambda_{k}$ autovalores não-nulos. Então $\mathfrak{g}_{0}(X)$ é subálgebra de Cartan se $X$ for regular.

Lema 1.7.5. Seja $\mathfrak{h}$ uma subálgebra de Cartan. Então, existe $X \in \mathfrak{h}$ tal que $\mathfrak{h}=\mathfrak{g}_{0}(X)$.

Dizemos que duas subálgebras de Cartan $\mathfrak{h}_{1}$ e $\mathfrak{h}_{2}$ são conjugadas se existe um automorfismo $\phi$ de $\mathfrak{g}$ tal que $\phi\left(\mathfrak{h}_{1}\right)=\mathfrak{h}_{2}$.

Teorema 1.7.6. Em álgebras de Lie sobre corpos algebricamente fechados as subálgebras de Cartan são conjugadas entre si.

Observação 1.7.7. Todas essas proposições e teoremas nos garantem que dada uma álgebra semi-simples $\mathfrak{g}$ um elemento regular $X \in \mathfrak{g}$, se tomarmos $\mathfrak{h}=\mathfrak{g}_{0}(X)$ uma subálgebra de Cartan, pela proposição 1.6 .2 e pelo teorema 1.7.4, como $\operatorname{ad}(X)$ é uma derivação, então

$$
\mathfrak{g}=\mathfrak{h} \oplus \mathfrak{g}_{\lambda_{1}} \oplus \ldots \oplus \mathfrak{g}_{\lambda_{k}}
$$

onde $\lambda_{i}$ são os pesos não-nulos de $\mathfrak{h}$, que nesse caso chamamos de raízes. 


\section{Capítulo 2}

\section{Representação Adjunta de Álgebras semi-simples}

\subsection{Representações de $\operatorname{sl}(2)$}

Para estudarmos as álgebras semi-simples iremos, inicialmente, analisar as representações irredutíveis da álgebra de Lie $s l(2)$ sobre um corpo $\mathbb{K}$ algebricamente fechado de característica zero. Seja $\{X, H, Y\}$ a base de $s l(2)$, onde

$$
X=\left(\begin{array}{ll}
0 & 1 \\
0 & 0
\end{array}\right), H=\left(\begin{array}{cc}
1 & 0 \\
0 & -1
\end{array}\right), Y=\left(\begin{array}{ll}
0 & 0 \\
1 & 0
\end{array}\right) .
$$

Essa base satisfaz:

$$
[H, X]=2 X,[H, Y]=-2 Y,[X, Y]=H .
$$

Tome $\rho: \operatorname{sl}(2) \rightarrow g l(V)$ uma representação irredutível de dimensão finita. Se $v \in V$ é um autovetor de $\rho(H)$ associado ao autovalor $\lambda$ então:

$$
\begin{gathered}
\rho[H, X] v=\rho(H) \rho(X) v-\rho(X) \rho(H) v \Rightarrow \\
\rho(H) \rho(X) v=\rho[H, X] v+\rho(X) \rho(H) v=\rho(2 X) v+\rho(X) \lambda v=(2+\lambda) \rho(X) v .
\end{gathered}
$$

Logo, se $\rho(X) v \neq 0$, então esse vetor é autovetor de $\rho(H)$ associado ao autovalor $\lambda+2$. Analogamente consegue-se mostrar que se $\rho(Y) v \neq 0$ então ele será autovetor de $\rho(H)$ associado ao autovalor $\lambda-2$. Assim aplicando essa formula $k$ vezes chegamos nas seguintes equações:

$$
\begin{aligned}
& \rho(H) \rho(X)^{k} v=(\lambda+2 k) \rho(X)^{k} v, \\
& \rho(H) \rho(Y)^{k} v=(\lambda-2 k) \rho(Y)^{k} v .
\end{aligned}
$$

Dessas observações segue o teorema: 
Teorema 2.1.1. Seja $\rho$ uma representação irredutivel de $\operatorname{sl(2)~em~} V \operatorname{com} \operatorname{dim} V=n+1$. Então, existe uma base $\left\{v_{0}, v_{1}, \ldots, v_{n}\right\}$ de $V$ tal que, para $i=0,1, \ldots, n$,

$$
\begin{aligned}
\rho(X) v_{i} & =i(n-i+1) v_{i-1} \\
\rho(H) v_{i} & =(n-2 i) v_{i} \\
\rho(Y) v_{i} & =v_{i+1}
\end{aligned}
$$

onde $v_{-1}=v_{n+1}=0$.

Demonstração: Seja $v$ um autovetor de $\rho(H)$ associado a $\lambda$. Assim, pelo que foi dito anteriormente, se $\rho(X)^{i} v \neq 0$ então esse vetor é autovetor de $\rho(H)$ associado ao autovalor $\lambda+2 i$. Como os autovetores estão associados a autovalores distintos eles formam um conjunto L.I.. Como V tem dimensão finita, existe $i_{0}>1$ tal que $\rho(X)^{i_{0}} v=0$ e $\rho(X)^{i_{0}-1} v \neq 0$. Fixe $v_{0}=\rho(X)^{i_{0}-1} v$ o autovetor associado ao autovalor $\lambda_{0}=\lambda+2 i_{0}$. Tome $v_{i}=\rho(Y)^{i} v_{0}$. Analogamente, o conjunto $\left\{v_{0}, v_{1}, \ldots, v_{k}\right\}$ é L.I., pois são autovetores associados aos diferentes autovalores $\lambda_{0}-2 i$, para $i=0,1, \ldots, k$, onde $k$ é tal que $v_{k+1}=0$. Definidos assim temos:

$$
\rho(X) v_{i}=i\left(\lambda_{0}-i+1\right) v_{i-1}
$$

Essa igualdade é provada por indução em $i$. Para $i=0$, por definição de $v_{0}, \rho(X) v_{0}=0$. Agora suponha a fórmula válida para $i-1$ e provemos para $i$. Temos:

$$
\rho(X) v_{i}=\rho(X) \rho(Y) v_{i-1}=\rho[X, Y] v_{i-1}+\rho(Y) \rho(X) v_{i-1}
$$

Mas os termos da soma do último membro da equação anterior são:

$$
\rho[X, Y] v_{i-1}=\rho(H) v_{i-1}=(\lambda-2(i-1)) v_{i-1}
$$

e pela hipótese de indução

$$
\rho(Y) \rho(X) v_{i-1}=\rho(Y)(i-1)\left(\lambda_{0}-(i-1)+1\right)=(i-1)\left(\lambda_{0}-i+2\right) v_{i-1} .
$$

Logo

$$
\begin{gathered}
\rho(X) v_{i}=(\lambda-2(i-1)) v_{i-1}+(i-1)\left(\lambda_{0}-i+2\right) v_{i-1}= \\
\left(\lambda_{0}-2 i+2+i \lambda_{0}-\lambda_{0}-i^{2}+2 i+i-2\right) v_{i-1}=i\left(\lambda_{0}-i+1\right) v_{i-1} .
\end{gathered}
$$

Portanto o espaço gerado por $\left\{v_{0}, v_{1}, \ldots, v_{k}\right\}$ é invariante por $\rho(X)$ e por construção também é invariante por $\rho(H)$ e $\rho(Y)$. Logo, como a representação é irredutível, $V$ é igual ao espaço gerado por $\left\{v_{0}, v_{1}, \ldots, v_{k}\right\}$ e portanto $k=n$. Assim para acabar a demonstração do teorema basta mostrar que $\lambda_{0}=n$. Por um lado temos $\rho(H) v_{n}=\left(\lambda_{0}-2 n\right) v_{n}$. Por outro lado,

$$
\begin{aligned}
& \rho(H) v_{n}=\rho[X, Y] v_{n}=\rho(X) \rho(Y) v_{n}-\rho(Y) \rho(X) v_{n} \\
& =-\rho(Y)\left(n\left(\lambda_{0}-n+1\right) v_{n-1}\right)=-n\left(\lambda_{0}-n+1\right) v_{n} .
\end{aligned}
$$

Assim, $\lambda_{0}-2 n=-n\left(\lambda_{0}-n+1\right)$, portanto $\lambda_{0}=n$. 
Observe que as matrizes de uma representação irredutível $\rho$ de $\operatorname{sl}(2)$ em $V$, onde $\operatorname{dim} V=n+1$, têm a forma

$$
\rho(X)=\left(\begin{array}{ccccccccccc}
0 & n & 0 & & 0 & 0 & 0 & 0 & 0 & 0 \\
0 & 0 & 2(n+1) & 0 & 0 & 0 & 0 & 0 & 0 \\
0 & 0 & 0 & 3(n+2) & 0 & 0 & 0 & 0 & 0 \\
0 & 0 & 0 & & 0 & & 0 & 0 & 0 & 0 \\
& & & & & & & \ddots & & & \\
0 & 0 & 0 & & 0 & 0 & 0 & 3(n-2) & 0 & 0 \\
0 & 0 & 0 & & 0 & 0 & 0 & 0 & 2(n-1) & 0 \\
0 & 0 & 0 & & 0 & 0 & 0 & 0 & 0 & n \\
0 & 0 & 0 & & 0 & 0 & 0 & 0 & 0 & 0
\end{array}\right)_{(n+1) \times(n+1)}
$$

Os autovalores de $\rho(H)$ formam uma progressão aritmética de razão -2 que começa em $n$ e termina em $-n$.

Teorema 2.1.2. Para cada $n \geq 0$ existe uma única representação irredutível de dimensão $n+1$ de sl(2)(a menos de isomorfismo) e essas representações cobrem todas as representações de dimensão finita de $\operatorname{sl}(2)$.

Demonstração: Dado um espaço vetorial $V$ de dimensão $n+1$, seja $\left\{v_{0}, v_{1}, \ldots, v_{n}\right\}$ uma base de $V$. Defina $\rho(X), \rho(H)$ e $\rho(Y)$ como nas expressões do teorema anterior. Assim $\rho$ é representação de $s l(2)$ em $V$. De fato, basta ver que o colchete é preservado: 


$$
\rho[H, Y] v_{i}=-2 \rho(Y) v_{i}=-2 v_{i+1}
$$

Por outro lado,

$$
\begin{aligned}
& {[\rho(H), \rho(Y)] v_{i}=\rho(H) \rho(Y) v_{i}-\rho(Y) \rho(H) v_{i}} \\
& \quad=\rho(H) v_{i+1}-\rho(Y)(n-2 i) v_{i}=(n-2(i+1)) v_{i+1}-(n-2 i) v_{i+1}=-2 v_{i+1}
\end{aligned}
$$

Logo

$$
\rho[H, Y] v_{i}=[\rho(H), \rho(Y)] v_{i}, \text { para todo } i .
$$

A prova de que os demais colchetes são preservados é análoga. Logo $\rho$ é representação e é irredutível, pois os subespaços invariantes por $\rho$ são invariantes por $\rho(H)$. Mas os únicos subespaços invariantes por $\rho(H)$ são os gerados pelos $v_{i}$ 's e esses subespaços não são invariantes por $\rho(X)$ e $\rho(Y)$. Logo não existem subespaços invariantes próprios, portanto a representação é irredutível. Isso prova a existência. A unicidade é garantida pois se tomarmos outro espaço $W$ de dimensão $n+1$ e uma representação irredutível $\alpha$. Então a tranformação linear que faz corresponder as bases dos espaços $V$ e $W$ será o operador de intercâmbio das representações $\rho$ e $\alpha$. Portanto essas representações serão isomorfas.

\subsection{Subálgebras de Cartan}

Seja $\mathfrak{g}$ uma álgebra de Lie semi-simples sobre $\mathbb{K}$ e $\mathfrak{h}$ uma subálgebra de Cartan de $\mathfrak{g}$. A álgebra se decompõe como

$$
\mathfrak{g}=\mathfrak{h} \oplus \mathfrak{g}_{\alpha_{1}} \oplus \mathfrak{g}_{\alpha_{2}} \oplus \ldots \oplus \mathfrak{g}_{\alpha_{k}}
$$

onde $\mathfrak{g}_{\alpha_{i}}$ são os subespaços de pesos da representação adjunta de $\mathfrak{h}$ em $\mathfrak{g}$ e $\alpha_{1}, \ldots, \alpha_{k}$ são os pesos não nulos. Essa decomposição é garantida pela observação 1.7.7. Esses pesos, como já foi dito, serão denominados raízes de $\mathfrak{h}$ em relação a g e a notação utilizada para esse conjunto será П. Os espaços $\mathfrak{g}_{\alpha_{i}}$ serão chamados espaços de raízes. Se representarmos a subálgebra $\mathfrak{h}$ em cada um dos $\mathfrak{g}_{\alpha_{i}}$, as matrizes serão da forma

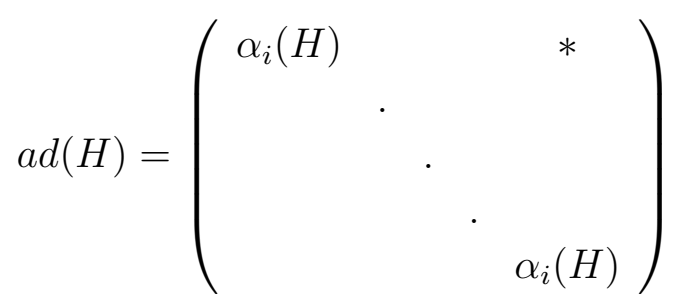

para todo $H \in \mathfrak{h}$. Vale também

$$
\left[\mathfrak{g}_{\alpha_{i}}, \mathfrak{g}_{\alpha_{j}}\right] \subset \mathfrak{g}_{\alpha_{i}+\alpha_{j}} .
$$


Essas duas últimas afirmações são garantidas pela proposição 1.6.2, pelo teorema 1.5.6 e por que $\mathfrak{h}$ é nilpotente.

Lema 2.2.1. Sejam $\alpha$ e $\beta$ pesos de $\mathfrak{h}$. Se $X \in \mathfrak{g}_{\alpha}$ e $Y \in \mathfrak{g}_{\beta}$, então

$$
\langle X, Y\rangle=\operatorname{tr}(\operatorname{ad}(X) \operatorname{ad}(Y))=0
$$

a menos que $\alpha=-\beta$.

Demonstração: Seja $z \in \mathfrak{g}_{\gamma}$. Então,

$$
\begin{gathered}
\operatorname{ad}(X) Z \in \mathfrak{g}_{\alpha+\gamma}, \\
\operatorname{ad}(Y) \operatorname{ad}(X) Z \in \mathfrak{g}_{\beta+\alpha+\gamma} .
\end{gathered}
$$

Se tomarmos uma base de $\mathfrak{g}$ como a união das bases de $\mathfrak{h}$ e $\mathfrak{g}_{\alpha_{i}}$, a matriz de $\operatorname{ad}(X) \operatorname{ad}(Y)$ em relação a essa base não tem elementos na diagonal, ou seja, nada irá contribuir para o traço, a menos que $\alpha+\beta=0$.

Corolário 2.2.2. 1. A restrição da forma de Cartan-Killing à subálgebra h é não degenerada.

2. Se $\alpha$ é raiz então $-\alpha$ é raiz .

3. Para todo $X \in \mathfrak{g}_{\alpha}$, existe $Y \in \mathfrak{g}_{-\alpha}$ tal que $\langle X, Y\rangle \neq 0$.

Demonstração: 1 . Seja $H \in \mathfrak{h}$. Como a forma de Cartan-Killing é não degenerada em $\mathfrak{g}$, existe $X \in \mathfrak{g}$ tal que $\langle H, X\rangle \neq 0$. Tome a decomposição de $\mathrm{X}$,

$$
X=H_{1}+X_{1}+\ldots+X_{k}
$$

onde $H_{1} \in \mathfrak{h}$ e $X_{i} \in \mathfrak{g}_{\alpha_{i}}$. Pelo lema anterior

$$
\left\langle H, X_{i}\right\rangle=0, \forall i=1, . ., k
$$

Portanto $\left\langle H, H_{1}\right\rangle \neq 0$.

2. Seja $X \in \mathfrak{g}_{\alpha}$. Então existe $Y \in \mathfrak{g}$ tal que $\langle X, Y\rangle \neq 0$ mas pelo lema $Y \in \mathfrak{g}_{-\alpha}$. Logo $\mathfrak{g}_{-\alpha} \neq 0$ e $-\alpha$ é raiz.

3. Se supormos que $\langle X, Y\rangle=0$ para todo $Y \in \mathfrak{g}_{-\alpha}$, então $\langle X, Z\rangle=0$ para todo $Z \in \mathfrak{g}$. Mas isso é um absurdo pois a forma de Cartan-Killing é não degenerada em $\mathfrak{g}$.

Proposição 2.2.3. . Para todo $H \in \mathfrak{h}$ e todo peso $\alpha$, ad $(H)_{\mid \mathfrak{g}_{\alpha}}=\alpha(H)$ id e as tranformações ad $(H)$ são simultaneamente diagonalizáveis. 
Demonstração: Já sabemos que

$$
a d(H)_{\mid \mathfrak{g}_{\alpha}}=\left(\begin{array}{cccc}
\alpha(H) & & & * \\
& \cdot & & \\
& \cdot & & \\
& & \cdot & \alpha(H)
\end{array}\right)
$$

Tome a decomposição $H=H_{S}+H_{N}$ (que existe pelo corolário 1.6.8) com $\operatorname{ad}\left(H_{S}\right)$ semi-simples, $\operatorname{ad}\left(H_{N}\right)$ nilpotente e $H, H_{S}, H_{N}$ comutanto dois a dois. Assim

$$
\operatorname{ad}\left(H_{N}\right)_{\mid \mathfrak{g} \alpha}=\left(\begin{array}{cccc}
0 & & & * \\
& \cdot & & \\
& \cdot & \\
& & \cdot & \\
& & & 0
\end{array}\right)
$$

Logo $\alpha\left(H_{N}\right)=0$, para todo $\alpha \in \Pi$. Portanto $H_{N}$ pertence ao auto-espaço associado ao autovalor 0 , que é $\mathfrak{h}$. Mas $\left\langle H_{N}, H^{\prime}\right\rangle=0$ para todo $H^{\prime} \in \mathfrak{h}$ pois $\operatorname{ad}\left(H_{N}\right) \operatorname{ad}\left(H^{\prime}\right)$ só tem zeros na diagonal. Isso implica que $H_{N}=0$, pois a forma de Cartan-Killing é não degenerada em $\mathfrak{h}$. Portanto $a d(H)=a d\left(H_{S}\right)$, que é diagonal.

Proposição 2.2.4. A subálgebra h é abeliana.

Demonstração: Pela proposição anterior

$$
\operatorname{ad}\left[H_{1}, H_{2}\right]=\left[\operatorname{ad}\left(H_{1}\right), \operatorname{ad}\left(H_{2}\right)\right]=0,
$$

para todo $H_{1}, H_{2} \in \mathfrak{h}$. Como a representação adjunta é fiel no caso das álgebras semisimples, $\left[H_{1}, H_{2}\right]=0$, e portanto $\mathfrak{h}$ é abeliana.

Proposição 2.2.5. O conjunto $\Pi$ das raízes gera o dual $\mathfrak{h}^{*}$ de $\mathfrak{h}$, isto é, $H=0$ se $\beta(H)=0$ para toda raiz $\beta$.

Demonstração: Pela proposição 2.2.3, ad $(\mathrm{H})=0$, se $\beta(H)=0$ para toda raiz $\beta \in \Pi$. Mas a representação adjunta é fiel, como já foi observado anteriormente, logo $\mathrm{H}=0$ se $\beta(H)=0$ para toda raiz $\beta \in \Pi$. Portanto, temos um conjunto de funcionais lineares de $\mathfrak{h}$ cujo único elemento no anulador é 0 , logo esse conjunto gera o dual $\mathfrak{h}^{*}$.

Agora já podemos definir a forma de Cartan-Killing em $\mathfrak{h}^{*}$. Pelo o fato de que ela é não degenerada em $\mathfrak{h}$, a aplicação $\varphi: \mathfrak{h} \longrightarrow \mathfrak{h}^{*}$ definida como:

$$
\varphi(H)=\langle H, \cdot\rangle
$$


é um isomofismo. Denotamos $\alpha_{H}(\cdot)=\langle H, \cdot\rangle$. Assim para cada $H \in \mathfrak{h}$ existe um único $\alpha \in \mathfrak{h}^{*}$ tal que $\varphi(H)=\alpha$. E reciprocamente, para cada $\alpha \in \mathfrak{h}^{*}$, existe único $H_{\alpha} \in \mathfrak{h}$ tal que

$$
\alpha(H)=\left\langle H_{\alpha}, H\right\rangle, \forall H \in \mathfrak{h} .
$$

Assim definimos a forma de Cartan-Killing em $\mathfrak{h}^{*}$ como

$$
\langle\alpha, \beta\rangle=\left\langle H_{\alpha}, H_{\beta}\right\rangle,
$$

se $\alpha$ e $\beta$ são dois funcionais lineares em $\mathfrak{h}$.

Pelo isomorfismo entre $\mathfrak{h}$ e $\mathfrak{h}^{*}$, as raízes $\alpha \in \Pi$ definem um número finito de elementos $H_{\alpha}$ que geram $\mathfrak{h}$, pois $\Pi$ gera $\mathfrak{h}^{*}$. O lema seguinte dará mais informações sobre a decomposição do espaço $\mathfrak{g}$ com soma dos espaços de raízes de $\mathfrak{h}$.

Lema 2.2.6. 1. Se $X \in \mathfrak{g}_{\alpha}$ e $Y \in \mathfrak{g}_{-\alpha}$, ent $\tilde{a} O[X, Y]=\langle X, Y\rangle H_{\alpha}$.

2. Para todo $X \in \mathfrak{g}_{\alpha}$, existe $Y \in \mathfrak{g}_{-\alpha}$ tal que $[X, Y]=H_{\alpha}$.

3. Sejam $\alpha$ e $\beta$ raízes. Então,

$$
\langle\beta, \alpha\rangle=q_{\beta \alpha}\langle\alpha, \alpha\rangle
$$

$\operatorname{com} q_{\beta \alpha} \in \mathbb{Q} .\left(\right.$ Em geral $\left.q_{\beta \alpha} \neq q_{\alpha \beta}\right)$.

4. Para todo $\alpha \in \Pi,\langle\alpha, \alpha\rangle \in \mathbb{Q}^{+}$.

5. $\operatorname{dim} \mathfrak{g}_{\alpha}=1$, para todo $\alpha \in \Pi$.

6. Os únicos múltiplos inteiros de uma raiz $\alpha$ que são raízes são $\alpha$ e $-\alpha$.

Demonstração: 1. Temos que $[X, Y] \in \mathfrak{g}_{\alpha-\alpha}=\mathfrak{h}$. Tome $H \in \mathfrak{h}$ arbitrário. Então

$$
\begin{aligned}
& \langle H,[X, Y]\rangle=\langle H, \operatorname{ad}(X) Y\rangle= \\
& -\langle\operatorname{ad}(X) H, Y\rangle=\langle\operatorname{ad}(H) X, Y\rangle= \\
& \alpha(H)\langle X, Y\rangle=\langle X, Y\rangle\left\langle H, H_{\alpha}\right\rangle=\left\langle H,\langle X, Y\rangle H_{\alpha}\right\rangle .
\end{aligned}
$$

Portanto

$$
\left\langle H,[X, Y]-\langle X, Y\rangle H_{\alpha}\right\rangle=0, \forall H \in \mathfrak{h} .
$$

Como a forma de Cartan-Killing é não degenerada em $\mathfrak{h}$,

$$
[X, Y]=\langle X, Y\rangle H_{\alpha} .
$$

2. Pelo corolário 2.2.2 existe $Y^{\prime} \in \mathfrak{g}_{-\alpha}$ tal que $\left\langle X, Y^{\prime}\right\rangle \neq 0$. Tome $Y=\frac{Y^{\prime}}{\left\langle X, Y^{\prime}\right\rangle}$ e temos

$$
[X, Y]=\langle X, Y\rangle H_{\alpha}=1 . H_{\alpha}
$$

3. Seja

$$
V=\ldots \oplus \mathfrak{g}_{\beta-2 \alpha} \oplus \mathfrak{g}_{\beta-\alpha} \oplus \mathfrak{g}_{\beta} \oplus \mathfrak{g}_{\beta+\alpha} \oplus \ldots
$$


onde $\mathfrak{g}_{\beta+k \alpha}=0$ se $\beta+k \alpha$ não é raiz. Essa soma direta é finita pois há um número finito de raízes. Sejam $X \in \mathfrak{g}_{\alpha}$ e $Y \in \mathfrak{g}_{-\alpha}$ tal que $[X, Y]=H_{\alpha}$. Pela definição de $V$, $a d(X) V \subset V$ e $\operatorname{ad}(Y) V \subset V$ e vale que

$$
\operatorname{ad}\left(H_{\alpha}\right)_{\mid V}=\operatorname{ad}([X, Y])_{\mid V}=\left[\operatorname{ad}(X)_{\mid V}, \operatorname{ad}(Y)_{\mid V}\right] .
$$

Logo $\operatorname{tr}\left(\operatorname{ad}\left(H_{\alpha}\right)_{\mid V}\right)=0$. Assim, se definirmos $d_{k}=\operatorname{dim} \mathfrak{g}_{\beta+k \alpha}$, temos

$$
\begin{aligned}
& 0=\operatorname{tr}\left(a d\left(H_{\alpha}\right)_{\mid V}\right)=\sum_{k} d_{k}(\beta+k \alpha)\left(H_{\alpha}\right)= \\
& \sum_{k} d_{k}(\langle\beta, \alpha\rangle+k\langle\alpha, \alpha\rangle)=\langle\beta, \alpha\rangle \sum_{k} d_{k}+\langle\alpha, \alpha\rangle \sum_{k} k d_{k},
\end{aligned}
$$

$\operatorname{logo}\langle\beta, \alpha\rangle=-\frac{\sum k d_{k}}{\sum d_{k}}\langle\alpha, \alpha\rangle$. É claro que o denominador dessa fração é não nulo pois ao menos um dos $\mathfrak{g}_{\beta+k \alpha} \neq 0$, portanto

$$
q_{\beta \alpha}=-\frac{\sum k d_{k}}{\sum d_{k}} \in \mathbb{Q}
$$

4. Pelo item 3., se $\langle\alpha, \alpha\rangle=0$, então $\langle\beta, \alpha\rangle=0$ para toda raiz $\beta$, mas isso é falso pois a forma é não degenerada em $\mathfrak{h}^{*}, \operatorname{logo}\langle\alpha, \alpha\rangle \neq 0$, para todo $\alpha$ pertencente a $\Pi$. Definindo $d_{\beta}=\operatorname{dim}_{\beta}$, temos:

$$
\begin{aligned}
\langle\alpha, \alpha\rangle=\left\langle H_{\alpha}, H_{\alpha}\right\rangle=\operatorname{tr}\left(\operatorname{ad}\left(H_{\alpha}\right)^{2}\right) & = \\
\sum_{\beta \in \Pi} d_{\beta} \beta\left(H_{\alpha}\right)^{2} & =\sum_{\beta \in \Pi} d_{\beta}\langle\beta, \alpha\rangle^{2}=\sum_{\beta \in \Pi} d_{\beta} q_{\beta \alpha}^{2}\langle\alpha, \alpha\rangle^{2},
\end{aligned}
$$

$\operatorname{assim}\langle\alpha, \alpha\rangle=\frac{1}{\sum d_{\beta} q_{\beta \alpha}^{2}} \in \mathbb{Q}^{+}$

5. Tome $X$ e $Y$ como no item 3., mas agora tome $V$ o espaço gerado por $Y, \mathfrak{h}$ e

$$
\sum_{k \geq 1} \mathfrak{g}_{k \alpha}
$$

Assim $V$ é invariante por $\operatorname{ad}(\mathrm{X})$ pois $a d(X) Y \in \mathfrak{h}$ e $\operatorname{ad}(X) \mathfrak{g}_{k \alpha} \subset \mathfrak{g}_{(k+1) \alpha}$. Ele é invariante por $\operatorname{ad}(\mathrm{Y})$, pois $\operatorname{ad}(Y) \mathfrak{g}_{k \alpha} \subset \mathfrak{g}_{(k-1) \alpha}$ e $\operatorname{ad}(Y) H=-\operatorname{ad}(H) Y=-\alpha(H) Y$, para todo $H \in \mathfrak{h}$. Analogamente ao item 3., como $H_{\alpha}=[X, Y], \operatorname{tr}\left(\operatorname{ad}\left(H_{\alpha}\right)_{\mid V}\right)=0$. Mas por outro lado, como $V=\left\langle\{Y\} \cup \mathfrak{h} \cup \sum \mathfrak{g}_{k \alpha}\right\rangle$,

$$
0=\operatorname{tr}\left(a d\left(H_{\alpha}\right)_{\mid V}\right)=-\alpha\left(H_{\alpha}\right)+\sum_{k \geq 0} d_{k} k \alpha\left(H_{\alpha}\right)
$$

onde $d_{k}=\operatorname{dim} \mathfrak{g}_{k \alpha} . \operatorname{Logo}$ 


$$
\langle\alpha, \alpha\rangle=\sum_{k \geq 0} k d_{k}\langle\alpha, \alpha\rangle
$$

Dividindo a equação por $\langle\alpha, \alpha\rangle$ temos

$$
1=d_{1}+2 d_{2}+3 d_{3}+\ldots
$$

com $d_{i} \geq 0$ para todo $i$. Portanto a igualdade só é satisfeita se $d_{1}=1$ e $d_{i}=0$ para todo $i \geq 2$.

6. Pelo que foi feito no item anterior $\operatorname{dim} \mathfrak{g}_{k \alpha}=0$ se $k \geq 2$. Logo $k \alpha$ só é raiz se $k= \pm 1$.

\subsection{Fórmula de Killing}

Agora, após a análise feita sobre as representações irredutíveis de $\operatorname{sl}(2)$, encontraremos subálgebras da álgebra semi-simples $\mathfrak{g}$ isomorfas a $\operatorname{sl}(2)$. Dada $\alpha$ uma raiz e $\mathfrak{h}(\alpha)$ o subespaço de $\mathfrak{h}$ gerado por $H_{\alpha}$. Então temos o seguinte resultado:

Proposição 2.3.1. A subágebra

$$
\mathfrak{g}(\alpha)=\mathfrak{g}_{-\alpha} \oplus \mathfrak{h}(\alpha) \oplus \mathfrak{g}_{\alpha}
$$

é isomorfa a sl(2).

Demonstração: Primeiramente $\mathfrak{g}(\alpha)$ é subálgebra pois $\left[\mathfrak{g}_{\alpha}, \mathfrak{g}_{-\alpha}\right] \subset \mathfrak{h}(\alpha),\left[\mathfrak{g}_{\alpha}, \mathfrak{h}(\alpha)\right] \subset$ $\mathfrak{g}_{\alpha}$ e $\left[\mathfrak{g}_{-\alpha}, \mathfrak{h}(\alpha)\right] \subset \mathfrak{g}_{-\alpha}$.

Seja $H_{\alpha}^{\prime} \in \mathfrak{h}(\alpha)$ definido por

$$
H_{\alpha}^{\prime}=\frac{2}{\langle\alpha, \alpha\rangle} H_{\alpha}
$$

Pelo lema 2.2.6, existem $X_{\alpha} \in \mathfrak{g}_{\alpha}$ e $Y_{-\alpha} \in \mathfrak{g}_{-\alpha}$ tais que

$$
\left\langle X_{\alpha}, Y_{-\alpha}\right\rangle=\frac{2}{\langle\alpha, \alpha\rangle}
$$

pois como existe $Y \in \mathfrak{g}_{-\alpha}$ tal que $\left\langle X_{\alpha}, Y\right\rangle=1$, tome por exemplo $Y_{-\alpha}=\frac{2}{\langle\alpha, \alpha\rangle} Y$.

Agora temos

$$
\begin{aligned}
& {\left[H_{\alpha}^{\prime}, X_{\alpha}\right]=\operatorname{ad}\left(H_{\alpha}^{\prime}\right) X_{\alpha}=\alpha\left(H_{\alpha}^{\prime}\right) X_{\alpha}=\left\langle H_{\alpha}^{\prime}, H_{\alpha}\right\rangle X_{\alpha}} \\
& =2 \frac{\left\langle H_{\alpha}, H_{\alpha}\right\rangle}{\langle\alpha, \alpha\rangle} X_{\alpha}=2 X_{\alpha},
\end{aligned}
$$




$$
\begin{gathered}
{\left[H_{\alpha}^{\prime}, Y_{-\alpha}\right]=\operatorname{ad}\left(H_{\alpha}^{\prime}\right) Y_{-\alpha}=\alpha\left(H_{\alpha}^{\prime}\right) Y_{-\alpha}=-2 Y_{-\alpha},} \\
{\left[X_{\alpha}, Y_{-\alpha}\right]=\left\langle X_{\alpha}, Y_{-\alpha}\right\rangle H_{\alpha}=H_{\alpha}^{\prime} .}
\end{gathered}
$$

Isso mostra que $\phi: \operatorname{sl}(2) \longrightarrow \mathfrak{g}(\alpha)$, dado por $\phi(X)=X_{\alpha}, \phi(Y)=Y_{-\alpha}$ e $\phi(H)=H_{\alpha}^{\prime}$ é um isomorfismo de álgebras de Lie. Aqui $\{X, H, Y\}$ é a base canônica de $\operatorname{sl}(2)$.

Observação 2.3.2. O isomorfismo entre as álgebras não é único pois os $X_{\alpha}$ e $Y_{-\alpha}$ não são únicos.

Observação 2.3.3. Para cada raiz $\alpha$ temos uma representação de $\operatorname{sl}(2)$ em $\mathfrak{g}$ pela composição de $\phi$ com a representação adjunta de $\mathfrak{g}(\alpha)$ em $\mathfrak{g}$. Com essas representações analisaremos mais detalhadamente os colchetes $\left[\mathfrak{g}_{\alpha}, \mathfrak{g}_{\beta}\right]$ e a forma $\langle\alpha, \beta\rangle$.

Definição 2.3.4. Considere $\alpha$ e $\beta$ duas raízes. A sequência de elementos de $\mathfrak{h}^{*}$

$$
\ldots, \beta-2 \alpha, \beta-\alpha, \beta, \beta+\alpha, \beta+2 \alpha, \ldots
$$

é denominada a $\alpha$-sequência iniciada em $\beta$.

Queremos encontrar quais desses elementos são pesos. A resposta é dada pelo teorema:

Teorema 2.3.5. Os elementos da $\alpha$-sequência iniciada em $\beta$ que são pesos formam um intervalo contendo $\beta$, isto é, existem inteiros $p, q \geq 0$ tais que

$$
\beta-p \alpha, \ldots, \beta-\alpha, \beta, \beta+\alpha, \ldots, \beta+q \alpha
$$

são os únicos pesos da forma $\beta+k \alpha$ com $k \in \mathbb{Z}$. Além do mais, vale o seguinte fórmula (de Killing)

$$
p-q=\frac{2\langle\beta, \alpha\rangle}{\langle\alpha, \alpha\rangle} .
$$

Demonstração: Suponha inicialmente que $\beta$ é múltiplo inteiro de $\alpha$ assim $\beta=0$ ou $\beta= \pm \alpha$. Logo a $\alpha$-sequência iniciada em $\beta$ fica

$$
-\alpha, 0, \alpha \text {. }
$$

Logo a fórmula de Killing vale nesse caso.

Agora suponha $\beta+k \alpha \neq 0$, para todo $k \in \mathbb{Z}$. Tome o subsespaço de $\mathfrak{g}$

$$
V_{\beta, \alpha}=\ldots \oplus \mathfrak{g}_{\beta-\alpha} \oplus \mathfrak{g}_{\beta} \oplus \mathfrak{g}_{\beta+\alpha} \oplus \ldots
$$


Essa soma é finita e a representação adjunta de $\mathfrak{g}(\alpha)$ em $\mathfrak{g}$ deixa $V_{\beta, \alpha}$ invariante, como já foi dito na parte 3. do lema 2.2.6. Logo podemos tomar a representação adjunta $\operatorname{ad}: \mathfrak{g}(\alpha) \longrightarrow g l\left(V_{\beta, \alpha}\right)$, e essa representação é irredutível. De fato, suponha que

$$
V_{\beta, \alpha}=V_{1} \oplus V_{2} \oplus \ldots \oplus V_{s}
$$

é uma decomposição de $V_{\beta, \alpha}$, com $V_{i}$ componentes irredutíveis. Seja $m_{i}=\operatorname{dim} V_{i}-1$. Pela classificação das representações irredutíveis de $s l(2) \cong \mathfrak{g}(\alpha)$, os autovalores de $a d\left(H_{\alpha}^{\prime}\right)$ são inteiros todos pares ou ímpares. Mas, por outro lado, os autovalores são

$$
(\beta+k \alpha)\left(H_{\alpha}^{\prime}\right)=\beta\left(H_{\alpha}^{\prime}\right)+k \alpha\left(H_{\alpha}^{\prime}\right)=2 \frac{\beta\left(H_{\alpha}\right)}{\langle\alpha, \alpha\rangle}+2 k \frac{\alpha\left(H_{\alpha}\right)}{\langle\alpha, \alpha\rangle}=2 \frac{\langle\beta, \alpha\rangle}{\langle\alpha, \alpha\rangle}+2 k
$$

logo o número $\frac{2\langle\beta, \alpha\rangle}{\langle\alpha, \alpha\rangle}$ é inteiro e os autovalores $(\beta+k \alpha)\left(H_{\alpha}^{\prime}\right)$ têm a mesma paridade que ele, pois

$$
2 \frac{\langle\beta, \alpha\rangle}{\langle\alpha, \alpha\rangle}=(\beta+k \alpha)\left(H_{\alpha}^{\prime}\right)-2 k \in \mathbb{Z} .
$$

Mas os autovalores são todos simples pois $\operatorname{dim} \mathfrak{g}_{\beta+k \alpha}=1, \operatorname{logo} s=1$. Pois se supormos $s>1$ então teríamos um autovalor $m_{i}$ de $V_{i}$, e mais, $m_{j}=m_{i}+2 k$ para algum $k>0$, o que implicaria que $m_{i}$ não é autovalor simples pois, se $v \in V_{j}$ é autovetor de $m_{j}$,

$$
a d\left(H_{\alpha}^{\prime}\right) v=m_{j} v=m_{i} v+2 k v=m_{i}\left(v+\frac{2 k}{m_{i}} v\right)
$$

e assim $m_{i}$ seria também autovalor de um vetor em $V_{j}$, o que é falso. Portanto $s=1$ e a representação é irredutível. Desse fato e do fato que $(\beta+k \alpha)\left(H_{\alpha}^{\prime}\right)$ varia de dois em dois quando se varia $k$ segue que

$$
V_{\beta, \alpha}=\mathfrak{g}_{\beta-p \alpha} \oplus \ldots \oplus \mathfrak{g}_{\beta-\alpha} \oplus \mathfrak{g}_{\beta} \oplus \mathfrak{g}_{\beta+\alpha} \oplus \ldots \oplus \mathfrak{g}_{\beta+q \alpha}
$$

De fato, se existisse $j, p<j<q$, tal que $\mathfrak{g}_{\beta+j \alpha}=0$, teríamos que $(\beta+j \alpha)\left(H_{\alpha}^{\prime}\right)$ não seria autovalor. Mas isso é absurdo pois, se $(\beta+(j-1) \alpha)\left(H_{\alpha}^{\prime}\right)$ é autovalor, então

$$
(\beta+j \alpha)\left(H_{\alpha}^{\prime}\right)=(\beta+(j-1) \alpha)\left(H_{\alpha}^{\prime}\right)+2
$$

que é autovalor. Assim o conjunto de pesos da forma $\beta+k \alpha$ é um intervalo.

Falta mostrar a fórmula. Por um lado, o maior autovalor de $\operatorname{ad}\left(H_{\alpha}^{\prime}\right)$ é

$$
(\beta+q \alpha)\left(H_{\alpha}^{\prime}\right)=\frac{2\langle\beta, \alpha\rangle}{\langle\alpha, \alpha\rangle}+2 q
$$

e por outro lado $\operatorname{dim} V_{\beta, \alpha}=q+p+1$. Logo

$$
p+q=\frac{2\langle\beta, \alpha\rangle}{\langle\alpha, \alpha\rangle}+2 q .
$$

Portanto 


$$
p-q=\frac{2\langle\beta, \alpha\rangle}{\langle\alpha, \alpha\rangle}
$$

Observação 2.3.6. Na fórmula de Killing os valores de p e q são diferentes se tomarmos a $\beta$-sequência iniciada em $\alpha$.

Observação 2.3.7. O inteiro

$$
\frac{2\langle\beta, \alpha\rangle}{\langle\alpha, \alpha\rangle}
$$

é denominado número de Killing associado às raízes $\alpha$ e $\beta$.

Proposição 2.3.8. Os únicos múltiplos de uma raiz $\alpha$ que são raízes são $\pm \alpha$ ou 0.

Demonstração: Suponha que $\beta=c \alpha$ é uma raiz, com $c \neq 0$. Logo

$$
\begin{aligned}
& \frac{2\langle\beta, \alpha\rangle}{\langle\alpha, \alpha\rangle}=2 c \\
& \frac{2\langle\beta, \alpha\rangle}{\langle\beta, \beta\rangle}=\frac{2}{c} .
\end{aligned}
$$

Assim temos dois números inteiros que o produto é igual a 4, mas isso implica que $\frac{2\langle\beta, \alpha\rangle}{\langle\alpha, \alpha\rangle}= \pm 1, \pm 2, \pm 4$ e portanto $\mathrm{c}= \pm \frac{1}{2}, \pm 1, \pm 2$. Mas não ocorre $c= \pm \frac{1}{2}$ ou \pm 2 , pois já foi provado que os únicos múltiplos inteiros de uma raíz $\alpha$ são $\pm \alpha$.

Proposição 2.3.9. Se $\alpha$ e $\beta$ são raízes tais que $\alpha+\beta$ é raiz, então

$$
\left[\mathfrak{g}_{\alpha}, \mathfrak{g}_{\beta}\right]=\mathfrak{g}_{\beta+\alpha} .
$$

Demonstração: Tomando $X_{\alpha} \in \mathfrak{g}(\alpha) \cong \operatorname{sl}(2)$, a expressão da representação irredutível de $\mathfrak{g}(\alpha)$ em $\mathfrak{g}$ mostra que

$$
\operatorname{ad}\left(X_{\alpha}\right) \mathfrak{g}_{\beta}=\mathfrak{g}_{\alpha+\beta} .
$$

De fato, $\operatorname{ad}\left(X_{\alpha}\right)$ leva a base de $\mathfrak{g}_{\beta}$ na base de $\mathfrak{g}_{\alpha+\beta}$. Logo todos os elementos de $\mathfrak{g}_{\alpha+\beta}$ são imagem de $a d\left(X_{\alpha}\right)$. Portanto temos a igualdade requerida na proposição. 


\subsection{Sistema simples de raízes}

O objetivo desta seção é encontrar um conjunto de raízes que seja base de $\mathfrak{h}^{*}$ visto como espaço vetorial sobre os racionais, e ainda mais, que os elementos de П sejam escritos como combinação dessa base com coeficientes inteiros. Esse conjunto será denominado sistema simples de raízes. Seja então $\mathfrak{h}_{\mathbb{Q}}$ o espaço:

$$
\mathfrak{h}_{\mathbb{Q}}=\left\{a_{1} H_{\alpha_{1}}+\ldots+a_{k} H_{\alpha_{k}}: a_{i} \in \mathbb{Q} \text { e } \alpha_{i} \in \Pi\right\}
$$

que é um espaço de dimensão finita sobre $\mathbb{Q}$, pois o conjunto de raízes é finito.

Proposição 2.4.1. $\operatorname{dimh}_{\mathbb{Q}}=\operatorname{dimh}$.

Demonstração: $\quad$ Primeiramente seja $\left\{\alpha_{1}, \ldots, \alpha_{l}\right\} \subset \Pi$ tal que $B=\left\{H_{\alpha_{1}}, \ldots, H_{\alpha_{l}}\right\}$ é base de $\mathfrak{h}$ sobre $\mathbb{K}$. Como o corpo $\mathbb{K}$ é de característica zero, ele contém os racionais e como o conjunto $B$ é L.I. sobre $\mathbb{K}$ é também L.I. sobre $\mathbb{Q}$. Assim $\operatorname{dim} \mathfrak{h}_{\mathbb{Q}} \geq \operatorname{dim} \mathfrak{h}$. Agora para mostrar a igualdade basta mostrar que $B$ gera $\mathfrak{h}_{\mathbb{Q}}$ sobre $\mathbb{Q}$. Seja $\alpha$ uma raiz qualquer, logo

$$
H_{\alpha}=a_{1} H_{\alpha_{1}}+\ldots+a_{l} H_{\alpha_{l}}, a_{i} \in \mathbb{K} .
$$

Agora, para cada $j=1, \ldots, l$, temos a equação

$$
\left\langle H_{\alpha}, H_{\alpha_{j}}\right\rangle=\sum_{i=1}^{l}\left\langle H_{\alpha_{i}}, H_{\alpha_{j}}\right\rangle a_{i} .
$$

Logo temos $l$ equações e $l$ incógnitas $a_{i}$. Mas assim esse sistema linear dá origem a uma matriz que é justamente a matriz da forma de Cartan-Killing em relação a base $B$, que é não-degenerada e portanto o sistema tem única solução. Como as entradas da matriz são todas racionais, pois os números $\left\langle H_{\alpha_{i}}, H_{\alpha_{j}}\right\rangle$ são todos racionais, a solução é racional. Portanto $a_{i} \in \mathbb{Q}$, para $i=1, \ldots, l$. Então $B$ gera $\mathfrak{h}_{\mathbb{Q}}$ sobre $\mathbb{Q}$, e as dimensões são iguais.

Proposição 2.4.2. A forma de Cartan-Killing restrita a $\mathfrak{h}_{\mathbb{Q}}$ é um produto interno.

Demonstração: Restringindo a forma de Cartan-Killing à $\mathfrak{h}_{\mathbb{Q}}$, definimos uma forma bilinear simétrica, pois o valor da forma em elementos de $\Pi$ assume valores em $\mathbb{Q}$. Basta mostrar então que ela é positiva definida. Tome $H \in \mathfrak{h}_{\mathbb{Q}}$, assim

$$
\langle H, H\rangle=\operatorname{tr}\left(a d(H)^{2}\right)=\sum_{\alpha \in \Pi} \alpha(H)^{2} \geq 0
$$

e

$$
\langle H, H\rangle=0 \Longleftrightarrow \alpha(H)=0, \forall \alpha \in \Pi \Longleftrightarrow H=0
$$


pois $\Pi$ gera $\mathfrak{h}^{*}$.

Esse espaço vetorial $\mathfrak{h}_{\mathbb{Q}}$ sobre os racionais foi construído para estudarmos a estrutura das álgebras semi-simples através da geometria dos elementos $H_{\alpha}$ ou das próprias raízes em $\mathfrak{h}_{\mathbb{Q}}^{*}$.

Antes ainda, iremos introduzir uma ordem lexicográfica nos espaços vetoriais racionais. Seja $V$ um espaço vetorial sobre $\mathbb{Q}$ e $\left\{v_{1}, \ldots, v_{l}\right\}$ uma base ordenada desse espaço. Escreva dois elementos $v, w \in V$ como combinação dos elementos da base

$$
\begin{aligned}
& v=a_{1} v_{1}+\ldots+a_{l} v_{l}, \\
& w=b_{1} v_{1}+\ldots+b_{l} v_{l} .
\end{aligned}
$$

Dizemos que $v \leq w$ se $v=w$ ou se $a_{i}<b_{i}$, para o primeiro íncide $i$ tal que $a_{i} \neq b_{i}$.

Definida assim a ordem lexicográfica em $V$ satifaz as propriedades que necessitaremos para provar a existência de um sistema simples de raízes.

Lema 2.4.3. Tomando a ordem lexicográfica dada pela base $\left\{v_{1}, \ldots, v_{l}\right\}$ de $V$, seja $\left\{w_{1}, \ldots, w_{m}\right\}$ um subconjunto de $V$ satisfazendo:

a) $w_{i}>0$ para todo $i=1, \ldots, m$,

b) $\left\langle w_{i}, w_{j}\right\rangle \leq 0$ para $i \neq j$.

Então $\left\{w_{1}, \ldots, w_{m}\right\}$ é um conjunto linearmente independente.

Demonstração: Suponha por absurdo que o conjunto seja linearmente dependente. Podemos supor que existem $a_{1}, \ldots, a_{m-1}$ tais que

$$
w_{m}=a_{1} w_{1}+\ldots+a_{m-1} w_{m-1} .
$$

Como $w_{m}>0$, pelo menos um dos $a_{i}>0$. De fato, se todos fossem negativos então $a_{i} w_{i}<0$ para todo $i=1, \ldots, m-1$, assim $w_{m}<0$. Seja então

$$
w_{m}=w_{m}^{+}+w_{m}^{-},
$$

onde $w_{m}^{+}$é a soma dos $a_{i} w_{i}$ tais que $a_{i}>0$ e $w_{m}^{-}$a soma dos $a_{i} w_{i}$ tais que $a_{i}<0$. Logo, por um lado, temos

$$
\left\langle w_{m}, w_{m}^{+}\right\rangle=\sum_{i} a_{i}\left\langle w_{m}, w_{i}\right\rangle \leq 0
$$

Por outro lado,

$$
\left\langle w_{m}, w_{m}^{+}\right\rangle=\left\langle w_{m}^{+}+w_{m}^{-}, w_{m}^{+}\right\rangle=\left|w_{m}^{+}\right|^{2}+\left\langle w_{m}^{-}, w_{m}^{+}\right\rangle
$$

e os termos do último membro da equação são positivos, pois $\left|w_{m}^{+}\right|^{2}$ é positivo e tomando $a_{i}$ os coeficientes positivos de $w_{m}$ e $a_{j}$ os coeficientes negativos, temos $a_{i} a_{j}<0$ e como $\left\langle w_{i}, w_{j}\right\rangle \leq 0$ então 


$$
\left\langle w_{m}^{-}, w_{m}^{+}\right\rangle=\sum a_{i} a_{j}\left\langle w_{i}, w_{j}\right\rangle>0 .
$$

Portanto o conjuto é L.I.

Definição 2.4.4. Fixada uma ordem lexicográfica no espaço vetorial racional $\mathfrak{h}_{\mathbb{Q}}^{*}$, uma raiz $\alpha \in \Pi$ é simples se

i) $\alpha>0$

ii) Não existem $\beta$ e $\gamma \in \Pi$ tais que $\beta, \gamma>0$ e $\alpha=\beta+\gamma$.

O conjunto das raízes simples será denotado $\Sigma$.

Lema 2.4.5. O conjunto $\Sigma$ é não vazio e linearmente independente.

Demonstração: Seja $\alpha$ uma raiz positiva minimal. Logo não existe $\beta$ raiz positiva tal que $\beta<\alpha$ (esse elemento existe pois o subconjunto das raízes positivas de $\Pi$ é finito e não vazio). Suponha, por absurdo, que $\alpha$ não é raiz simples. Assim existem $\beta$ e $\gamma \in \Pi$ tais que $\beta, \gamma>0$ e $\alpha=\beta+\gamma$, mas assim $0<\beta<\alpha$ o que implicaria que $\alpha$ não é raiz minimal. Logo $\alpha \in \Sigma$ e portanto $\Sigma \neq \emptyset$. Agora para mostrarmos que $\Sigma$ é L.I. utilizaremos o lema 2.4 .3 provando que se $\alpha$ e $\beta$ estão em $\Sigma, \alpha \neq \beta$, então $\langle\alpha, \beta\rangle \leq 0$. Primeiro observe que $\beta-\alpha$ não é raiz, pois se fosse, como

$$
\beta=\alpha+(\beta-\alpha)
$$

então $\beta-\alpha \leq 0$, pois $\beta$ é simples. Por outro lado, como

$$
\alpha=\beta+(\alpha-\beta)
$$

então $\beta-\alpha \geq 0$, pois $\alpha$ é simples. Logo, na $\alpha$-sequência iniciada em $\beta, p=0$. Assim

$$
\frac{2\langle\beta, \alpha\rangle}{\langle\alpha, \alpha\rangle}=-q \leq 0
$$

e portanto $\langle\beta, \alpha\rangle \leq 0$ se $\alpha \neq \beta$ são raízes simples. Pelo lema 2.4 .3 o conjunto $\Sigma$ é linermente independente.

O conjunto finito das raízes simples será escrito como

$$
\Sigma=\left\{\alpha_{1}, \ldots, \alpha_{l}\right\}
$$

Lema 2.4.6. Seja $\beta \in \Pi \operatorname{com} \beta>0$. Então $\beta$ se escreve de maneira única como

$$
\beta=n_{1} \alpha_{1}+\ldots+n_{l} \alpha_{l},
$$

com $n_{i} \in \mathbb{Z}$ e $n_{i} \geq 0$, para $i=1, \ldots, l$. 
Demonstração: Se $\beta \in \Sigma$ não há o que fazer. Senão existem $\beta_{1}$ e $\beta_{2}$ tais que $\beta=\beta_{1}+\beta_{2}$. Se $\beta_{1}, \beta_{2} \in \Sigma$ acabou a demonstração. Senão, para cada $\beta_{i}$, existem $\beta_{i 1}, \beta_{i 2}>0$, tais que

$$
\beta_{i}=\beta_{i 1}+\beta_{i 2} \quad i=1,2 .
$$

Note que $\beta_{i j}<\beta_{i}, i=1,2$. Se $\beta_{i j}$ são simples temos o resultado, se não são simples continua-se esse processo até que todas as raízes sejam tais que não não existe nenhuma raiz positiva menor que as mesmas. Assim essas raízes serão simples e $\beta$ é uma combinação linear dessas raízes com coeficientes inteiros.

Corolário 2.4.7. a) Seja $\gamma>0$ raiz que não é simples. Então, existe $\alpha \in \Sigma$ tal que $\langle\gamma, \alpha\rangle>0$ e $\gamma-\alpha$ é raiz positiva.

b) Toda raiz positiva pode ser escrita como

$$
\gamma=\alpha_{i_{1}}+\ldots+\alpha_{i_{k}}
$$

com $\alpha_{i_{j}}$ raiz simples e as somas parciais

$$
\alpha_{i_{1}}+\ldots+\alpha_{i_{s}}
$$

$s=1, \ldots, k$ são raízes

Demonstração: a)Se para toda raiz simples $\alpha$ tivermor $\langle\gamma, \alpha\rangle \leq 0$ então, pelo lema 2.4.3, o conjunto $\Sigma \cup\{\gamma\}$ é L.I. o que contradiz o lema 2.4.6. Logo existe $\alpha$ tal que $\langle\gamma, \alpha\rangle>0$. Assim, pela fómula de Killing, na $\alpha$-sequência iniciada em $\gamma$ temos $p>0$, pois

$$
p-q=\frac{2\langle\gamma, \alpha\rangle}{\langle\alpha, \alpha\rangle}>0
$$

$\operatorname{Logo} \gamma-\alpha$ é raiz. É raiz positiva, pois se supormos que é negativa, então

$$
\gamma-\alpha=a_{1} \alpha_{1}+\ldots+a_{n} \alpha_{n}+c \alpha
$$

$\operatorname{com} a_{i}, c \leq 0, i=1, \ldots, n$. Logo

$$
\gamma=a_{1} \alpha_{1}+\ldots+a_{n} \alpha_{m}+(c+1) \alpha
$$

Como $\gamma$ é raiz positiva, teríamos $a_{i}=0, i=1, \ldots, n$. Logo $\gamma$ seria um múltiplo de $\alpha$, mas isso é falso pois $\gamma$ não é simples.

b) Novamente, se $\gamma$ é raiz simples não há nada a fazer. Se $\gamma$ não é raiz simples então existe $\alpha \in \Sigma$ tal que $\gamma-\alpha$ é raiz positiva. Como $\gamma=(\gamma-\alpha)+\alpha$ se $\gamma-\alpha$ é raiz simples temos o resultado. Se não for raiz simples então aplicamos o mesmo argumento para a raiz positiva $\gamma-\alpha$, e o resultado segue indutivamente. 
Como conclusão desses últimos lemas temos que:

1) $\Sigma$ é uma base de $\mathfrak{h}_{\mathbb{Q}}^{*}$;

2) Para todo $\beta \in \Pi$

$$
\beta=n_{1} \alpha_{1}+\ldots+n_{l} \alpha_{l}
$$

com todos coeficientes inteiros e de mesmo sinal.

A afirmação 1) vem do fato que já provamos que o conjunto $\Pi$ gera $\mathfrak{h}_{\mathbb{Q}}^{*}$ e pelo lema 2.4.6, $\Sigma$ gera П. Para a afirmação 2 ), se $\beta$ é raiz positiva, então ele é combinação linear com coefinientes inteiros não negativos de elementos de $\Sigma$. Se $\beta$ é raiz negativa então $-\beta$ é positiva e portanto $\beta$ é combinação linear com coeficientes inteiros não positivos.

Definição 2.4.8. Um subconjunto $\Sigma=\left\{\alpha_{1}, \ldots, \alpha_{l}\right\}$ de $\Pi$, satisfazendo 1) e 2) acima é denominado sistema simples de raízes.

Observação 2.4.9. Não existe um único sistema simples de raízes. Se $\Sigma$ é um sistema simples de raízes, então $\left\{-\alpha_{1}, \ldots,-\alpha_{l}\right\}$ também é um sistema simples de raízes.

\subsection{Matrizes de Cartan}

Foi mostrado que se tivermos um sistema simples de raízes $\Sigma$ então todas as possíveis raízes de uma álgebra de Lie $\mathfrak{g}$ são combinações lineares com coeficientes inteiros todos positivos ou todos negativos. Um dos tópicos dessa seção será definir quando uma soma de elementos de $\Sigma$ é uma raiz. Isso será feito utilizando a fórmula de Killing, para isso primeiro precisamos de uma definição que irá diferenciar as raízes positivas pela quantidade de raízes simples que aparece em sua expressão.

Definição 2.5.1. Seja $\Sigma=\left\{\alpha_{1}, \ldots, \alpha_{l}\right\}$ o sistema simples fixado. Se $\beta$ é uma raiz positiva tal que

$$
\beta=m_{1} \alpha_{1}+\ldots+m_{l} \alpha_{l}, m_{i} \in \mathbb{Z}^{+},
$$

então o número inteiro positivo $m_{1}+\ldots+m_{l}$ é denominado a altura de $\beta$.

As raízes de altura um são as próprias raízes simples.

As raízes de altura dois são da forma $\alpha_{i}+\alpha_{j}$, com $i \neq j$. Para saber se $\alpha_{i}+\alpha_{j}$ é realmente raiz temos que analisar a $\alpha_{i}$-sequência iniciada em $\alpha_{j}$

$$
\alpha_{j}-p \alpha_{i}, \ldots, \alpha_{j}+q \alpha_{i}
$$

Como $\alpha_{j}-\alpha_{i}$ não é raiz (pois todos os coeficientes de uma raiz tem o mesmo sinal) sabemos que $p=0$. Logo, pela fórmula de Killing

$$
-q=\frac{2\left\langle\alpha_{i}, \alpha_{j}\right\rangle}{\left\langle\alpha_{i}, \alpha_{i}\right\rangle}
$$


Assim,

$$
q>0 \Leftrightarrow \frac{2\left\langle\alpha_{i}, \alpha_{j}\right\rangle}{\left\langle\alpha_{i}, \alpha_{i}\right\rangle}<0
$$

portanto

$$
\alpha_{i}+\alpha_{j} \in \Pi \Leftrightarrow \frac{2\left\langle\alpha_{i}, \alpha_{j}\right\rangle}{\left\langle\alpha_{i}, \alpha_{i}\right\rangle}<0
$$

Logo, para encontrarmos as raízes de altura dois, basta olhar os valores de $\frac{2\left\langle\alpha_{i}, \alpha_{j}\right\rangle}{\left\langle\alpha_{i}, \alpha_{i}\right\rangle}$.

As raízes $\beta$ de altura três, pelo corolário 2.4.7, são da forma $\alpha+\alpha_{k}$ com $\alpha$ de altura dois e $\alpha_{k} \in \Sigma$, ou seja, $\beta=\alpha_{i}+\alpha_{j}+\alpha_{k}$. Vamos analisar a $\alpha_{k}$-sequência iniciada em $\alpha_{i}+\alpha_{j}$. Temos

$$
p-q=\frac{2\left\langle\alpha_{i}+\alpha_{j}, \alpha_{k}\right\rangle}{\left\langle\alpha_{k}, \alpha_{k}\right\rangle} .
$$

Assim há duas situações:

a) $i \neq j \neq k$. Neste caso $p=0$ pois $\alpha_{i}+\alpha_{j}-\alpha_{k}$ não é raiz. Assim $\alpha_{i}+\alpha_{j}+\alpha_{k}$ é raiz se, e somente se, $q>0$. Isso ocorre se, e somente se,

$$
\frac{2\left\langle\alpha_{i}, \alpha_{k}\right\rangle}{\left\langle\alpha_{k}, \alpha_{k}\right\rangle}<0 \quad \text { ou } \quad \frac{2\left\langle\alpha_{j}, \alpha_{k}\right\rangle}{\left\langle\alpha_{k}, \alpha_{k}\right\rangle}<0
$$

pois

$$
-q=\frac{2\left\langle\alpha_{i}, \alpha_{k}\right\rangle}{\left\langle\alpha_{k}, \alpha_{k}\right\rangle}+\frac{2\left\langle\alpha_{j}, \alpha_{k}\right\rangle}{\left\langle\alpha_{k}, \alpha_{k}\right\rangle}
$$

e os termos do lado direito da equação são sempre menores ou iguais a zero.

b) $k=i$ ou $k=j$. Se $k=j$, então a $\alpha_{k}$-sequência iniciada em $\alpha_{i}+\alpha_{j}$ é parte, na verdade, da $\alpha_{j}$-sequência iniciada em $\alpha_{i}$. Analogamente, para decidir se $\alpha_{i}+2 \alpha_{j}$ é raiz temos que saber se

$$
\frac{2\left\langle\alpha_{i}, \alpha_{j}\right\rangle}{\left\langle\alpha_{j}, \alpha_{j}\right\rangle}<0
$$

Esse argumento se estende ao caso geral por indução. Pois, pelo corolário 2.4.7, dada uma raiz $\beta$ de altura $n+1$, ela é da forma $\alpha+\alpha_{k} \operatorname{com} \alpha$ raiz de altura $n$ e $\alpha_{k} \in \Sigma$. Novamente a fórmula de Killing nos diz quando essa soma é uma raiz. Olhando a $\alpha_{k}$-sequência iniciada em $\alpha$ temos

$$
p-q=\frac{2\left\langle\alpha, \alpha_{k}\right\rangle}{\left\langle\alpha_{k}, \alpha_{k}\right\rangle}
$$

Por indução conhecemos $p$ e $q$ e sabemos se $\alpha-\alpha_{k}, \alpha-2 \alpha_{k}, \ldots$, são raízes positivas e de altura menor que $n$. Se

$$
\alpha=n_{1} \alpha_{1}+\ldots+n_{l} \alpha_{l}
$$

então

$$
\frac{2\left\langle\alpha, \alpha_{k}\right\rangle}{\left\langle\alpha_{k}, \alpha_{k}\right\rangle}=n_{1} \frac{2\left\langle\alpha, \alpha_{1}\right\rangle}{\left\langle\alpha_{1}, \alpha_{1}\right\rangle}+\ldots+n_{l} \frac{2\left\langle\alpha, \alpha_{l}\right\rangle}{\left\langle\alpha_{l}, \alpha_{l}\right\rangle}
$$


Assim, sabemos se $q>0$, ou seja, se $\alpha+\alpha_{k}$ é raiz, se sabemos os números de Killing associados a $\alpha$ e $\alpha_{i} \in \Sigma$.

Portanto, pela discussão feita acima, os números de Killing associados aos elementos do sistema simples de raízes determinam todas as raízes de uma subálgebra de Cartan $\mathfrak{h} \subset \mathfrak{g}$. Consequentemente toda estrutura da álgebra semi-simples $\mathfrak{g}$. Esses números são colocados em forma de matriz:

$$
C=\left(\frac{2\left\langle\alpha_{i}, \alpha_{j}\right\rangle}{\left\langle\alpha_{i}, \alpha_{i}\right\rangle}\right)_{i, j} .
$$

Esta matriz é uma matriz $l \times l$ e recebe o nome de Matriz de Cartan do sistema simples de raízes. Os elementos da diagonal são todos iguais a 2 e os elementos restantes são inteiros não positivos.

Observação 2.5.2. Como a forma de Cartan-Killing restrita a h é um produto interno, podemos falar entre ângulos de elementos de $\Pi$, pois $\langle\alpha, \beta\rangle=\left\langle H_{\alpha}, H_{\beta}\right\rangle=\left|H_{\alpha}\right|\left|H_{\beta}\right| \cos \theta$.

Proposição 2.5.3. Sejam $\alpha$ e $\beta$ raízes simples.

a) Se $\theta$ denota o ângulo entre $\alpha$ e $\beta$ então,

$$
\cos \theta=0, \pm 1, \pm \frac{\sqrt{3}}{2}, \pm \frac{\sqrt{2}}{2}, \pm \frac{1}{2}
$$

isto é, $\theta=\frac{k \pi}{6}$ ou $\frac{k \pi}{4}$.

b) Os possíveis valores para os números de Killing são

$$
\frac{2\langle\alpha, \beta\rangle}{\langle\alpha, \alpha\rangle}=0, \pm 1, \pm 2, \pm 3
$$

Demonstração: a) Temos $\langle\alpha, \beta\rangle^{2}=\langle\alpha, \alpha\rangle\langle\beta, \beta\rangle \cos ^{2} \theta$. Logo,

$$
\frac{2\langle\alpha, \beta\rangle}{\langle\alpha, \alpha\rangle} \frac{2\langle\alpha, \beta\rangle}{\langle\beta, \beta\rangle}=4 \cos ^{2} \theta \text {. }
$$

Como $0 \leq \cos ^{2} \theta \leq 1$ e os termos do lado esquerdo da equação são inteiros, então

$$
4 \cos ^{2} \theta=0,1,2,3,4
$$

e portanto

$$
\cos \theta=0, \pm 1, \pm \frac{\sqrt{3}}{2}, \pm \frac{\sqrt{2}}{2}, \pm \frac{1}{2} .
$$

b) Pelo item anterior

$$
\frac{2\langle\alpha, \beta\rangle}{\langle\alpha, \alpha\rangle} \frac{2\langle\alpha, \beta\rangle}{\langle\beta, \beta\rangle}=0,1,2,3,4
$$

e isso implica que

$$
\frac{2\langle\alpha, \beta\rangle}{\langle\alpha, \alpha\rangle}=0, \pm 1, \pm 2, \pm 3, \pm 4
$$


Se supormos que

$$
\frac{2\langle\alpha, \beta\rangle}{\langle\alpha, \alpha\rangle}= \pm 4
$$

então $\cos \theta= \pm 1$. Logo $\theta=0$ ou $\pi$ e $\alpha$ seria múltiplo de $\beta$, ou seja, $\alpha= \pm \beta$ e assim

$$
\frac{2\langle\alpha, \beta\rangle}{\langle\alpha, \alpha\rangle}= \pm 2
$$

uma contradição. Portanto temos o resultado.

Observação 2.5.4. Se $\theta$ é o ângulo entre $\alpha_{i}$ e $\alpha_{j}$ raízes simples, então

- $\theta=0$ se $\alpha_{i}=\alpha_{j}$

- $\theta=90^{\circ}, 120^{\circ}, 135^{\circ}, 150^{\circ}$ se $\alpha_{i} \neq \alpha_{j}$.

Proposição 2.5.5. Seja $C=\left(c_{i j}\right)$ a matriz de Cartan de um sistema simples de raízes.

Então,

1. $c_{i i}=2$ para todo $i$,

2. $c_{i j}=0,-1,-2$ ou -3 , para $i \neq j$,

3. $c_{j i}=-1$ se $c_{i j}=-2$ ou -3 e

4. $c_{i j}=0$ se e somente se $c_{j i}=0$.

Demonstração: 1. Óbvio

2. Como os números de Killing são menores ou iguais a zero, pois $\langle\alpha, \beta\rangle \leq 0$ para todo $\alpha$ e $\beta \in \Sigma$, segue da proposição anterior.

3. Foi visto na proposição anterior que

$$
\frac{2\left\langle\alpha_{i}, \alpha_{j}\right\rangle}{\left\langle\alpha_{i}, \alpha_{i}\right\rangle} \frac{2\left\langle\alpha_{i}, \alpha_{j}\right\rangle}{\left\langle\alpha_{j}, \alpha_{j}\right\rangle} \leq 3
$$

Logo, se

$$
\frac{2\left\langle\alpha_{i}, \alpha_{j}\right\rangle}{\left\langle\alpha_{i}, \alpha_{i}\right\rangle}=-2 \text { ou }-3,
$$

então

$$
\frac{2\left\langle\alpha_{i}, \alpha_{j}\right\rangle}{\left\langle\alpha_{j}, \alpha_{j}\right\rangle}=-1
$$

4. Temos $c_{i j}=0=\frac{2\left\langle\alpha_{i}, \alpha_{j}\right\rangle}{\left\langle\alpha_{i}, \alpha_{i}\right\rangle}$ se, e somente se, $c_{j i}=\frac{2\left\langle\alpha_{i}, \alpha_{j}\right\rangle}{\left\langle\alpha_{j}, \alpha_{j}\right\rangle}=0$. 


\subsection{Diagramas de Dynkin}

Nesta seção introduziremos o diagrama de Dynkin, que é um grafo que contém todas as informações de uma matriz de Cartan, mas tem uma notação mais sucinta. Esse diagrama é definido, como uma matriz de Cartan, a partir de um sistema simples de raízes fixado $\Sigma=\left\{\alpha_{1}, \ldots, \alpha_{l}\right\}$.

O diagrama contém $l$ pontos (vértices) representando cada uma das raízes simples. Os vértices são ligados ou não por um, dois ou três segmentos (arestas) de acordo com as seguintes regras:

1. Se

$$
\frac{2\left\langle\alpha_{i}, \alpha_{j}\right\rangle}{\left\langle\alpha_{i}, \alpha_{i}\right\rangle}=\frac{2\left\langle\alpha_{i}, \alpha_{j}\right\rangle}{\left\langle\alpha_{j}, \alpha_{j}\right\rangle}=0
$$

não existe ligação:

$$
\stackrel{\circ}{\alpha_{i}} \stackrel{\circ}{\alpha_{j}}
$$

Nesse caso o ângulo $\theta$ entre as raízes é $90^{\circ}$, pois $4 \cos ^{2} \theta=0$ implica $\cos \theta=0$.

2. Se

$$
\frac{2\left\langle\alpha_{i}, \alpha_{j}\right\rangle}{\left\langle\alpha_{i}, \alpha_{i}\right\rangle}=\frac{2\left\langle\alpha_{i}, \alpha_{j}\right\rangle}{\left\langle\alpha_{j}, \alpha_{j}\right\rangle}=-1
$$

$\alpha_{i}$ e $\alpha_{j}$ são ligadas por um segmento:

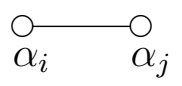

Nesse caso o ângulo $\theta$ entre as raízes é $120^{\circ}$, pois $4 \cos ^{2} \theta=-1$ implica $\cos \theta=-\frac{1}{2}$.

3. Se

$$
\frac{2\left\langle\alpha_{i}, \alpha_{j}\right\rangle}{\left\langle\alpha_{i}, \alpha_{i}\right\rangle} \text { ou } \frac{2\left\langle\alpha_{i}, \alpha_{j}\right\rangle}{\left\langle\alpha_{j}, \alpha_{j}\right\rangle}
$$

é igual a -2 (respectivamente -3), os vértices $\alpha_{i}$ e $\alpha_{j}$ são ligados por dois (respectivamente 3) segmentos:

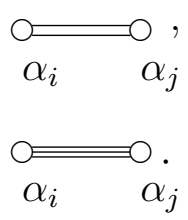

Nesse caso o ângulo $\theta$ entre as raízes é $135^{\circ}$ (respectivamente $150^{\circ}$ ), pois $4 \cos ^{2} \theta=2$ implica $\cos \theta= \pm \frac{\sqrt{2}}{2}$ (respectivamente $4 \cos ^{2} \theta=3$ implica $\cos \theta= \pm \frac{\sqrt{3}}{2}$ ) . 
Essas instruções dão as informações para que utilizemos a matriz de Cartan para encontrarmos o diagrama de Dynkin, no caso de um diagrama não orientado.

No entanto, quando a ligação é feita por dois ou três segmentos, não fica claro qual das entradas

$\mathrm{Ou}$

$$
c_{i j}=\frac{2\left\langle\alpha_{i}, \alpha_{j}\right\rangle}{\left\langle\alpha_{i}, \alpha_{i}\right\rangle}
$$

$$
c_{j i}=\frac{2\left\langle\alpha_{i}, \alpha_{j}\right\rangle}{\left\langle\alpha_{j}, \alpha_{j}\right\rangle}
$$

da matriz de Cartan é -2 ou -3. Para distinguir isso, orienta-se a ligação na direção da raiz $\alpha_{j}$ se

$$
\begin{aligned}
& c_{j i}=\frac{2\left\langle\alpha_{i}, \alpha_{j}\right\rangle}{\left\langle\alpha_{j}, \alpha_{j}\right\rangle}=-2 \text { ou }-3 .
\end{aligned}
$$

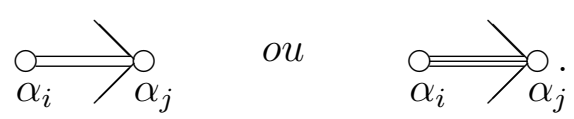

Orienta-se a ligação na direção da raiz $\alpha_{i}$ se

$$
c_{i j}=\frac{2\left\langle\alpha_{i}, \alpha_{j}\right\rangle}{\left\langle\alpha_{i}, \alpha_{i}\right\rangle}=-2 \text { ou }-3 .
$$

Assim temos as informações necessárias para que, do diagrama de Dynkin, encontremos a matriz de Cartan, concluindo assim a correspondência.

\subsection{Exemplo de Álgebra Semi-Simples}

Analisaremos nessa seção os conceitos apresentados durante todo o capítulo aplicado em uma álgebra de Lie concreta. O exemplo que iremos analisar é a álgebra clássica das matrizes $n \times n$ de traço nulo, denotada $\operatorname{sl}(n)$. É fácil verificar que as matrizes diagonais de traço nulo é uma subálgebra de Cartan de $\operatorname{sl}(n)$, que denotaremos aqui como h. Seja $E_{i j}=\left(a_{r s}\right)$, para $i, j=1,2, \ldots, n$, a matriz $n \times n$ cuja única entrada não nula é $a_{i j}=1$. O conjunto das matrizes $E_{i j}$ e $E_{i i}-E_{j j}, i \neq j$, é uma base de $s l(n)$. Dado um elemento $H \in \mathfrak{h}$, escrevemos

$$
H=\operatorname{diag}\left\{a_{1}, a_{2}, \ldots, a_{n}\right\}
$$

com $a_{1}+\ldots+a_{n}=0$. Assim, tomando a aplicação adjunta de $H$, temos

$$
\operatorname{ad}(H)\left(E_{i j}\right)=\left[H, E_{i j}\right]=H E_{i j}-E_{i j} H=\left(a_{i}-a_{j}\right) E_{i j} .
$$

Esta igualdade nos diz que os $E_{i j}$ são auto-vetores de $\operatorname{ad}(H)$ associados aos autovalores $\left(a_{i}-a_{j}\right)$. Isso mostra que as raízes de $\mathfrak{h}$ são os funcionais $\alpha_{i j}=\lambda_{i}-\lambda_{j}, i \neq j$, onde

$$
\begin{aligned}
\lambda_{i}: \underset{h}{\mathfrak{h}} & \longrightarrow \mathbb{K} \\
\quad \operatorname{diag}\left\{a_{1}, \ldots, a_{n}\right\} & \longrightarrow a_{i} .
\end{aligned}
$$


Os espaços de raízes são os espaços gerados por $E_{i j}, i \neq j$. Agora analisando a forma de Cartan-Killing temos,

$$
\begin{aligned}
\langle H, H\rangle= & \operatorname{tr}(\operatorname{ad}(H) a d(H))=\operatorname{tr}\left(\operatorname{ad}(H)^{2}\right)=\sum_{i \neq j}\left(a_{i}-a_{j}\right)^{2}= \\
& 2 \sum_{i<j}\left(a_{i}-a_{j}\right)^{2}=2 \sum_{i<j}\left(a_{i}^{2}+a_{j}^{2}\right)-4 \sum_{i<j} a_{i} a_{j}=2(n-1) \sum_{i=1}^{n} a_{i}^{2}-4 \sum_{i<j} a_{i} a_{j} .
\end{aligned}
$$

Mas

$$
-4 \sum_{i<j} a_{i} a_{j}=2 \sum_{i=1}^{n} a_{i}^{2}
$$

pois $\sum_{i=1}^{n} a_{i}=0$. Portanto,

$$
\langle H, H\rangle=2(n-1) \sum_{i=1}^{n} a_{i}^{2}-4 \sum_{i<j} a_{i} a_{j}=2(n-1) \sum_{i=1}^{n} a_{i}^{2}+2 \sum_{i=1}^{n} a_{i}^{2}=2 n \sum_{i=1}^{n} a_{i}^{2} .
$$

Esta última igualdade e a forma de polarização que relaciona uma forma quadrática com a forma bilinear associada, mostram que

$$
\left\langle H, H^{\prime}\right\rangle=2 n\left(a_{1} b_{1}+\ldots+a_{n} b_{n}\right)
$$

onde $H^{\prime}=\operatorname{diag}\left\{b_{1}, \ldots, b_{n}\right\}$.

Voltando a notação que associa a cada raiz $\alpha \in \Pi$ seu elemento $H_{\alpha} \in \mathfrak{h}$, se $\alpha_{i j}=\lambda_{i}-\lambda_{j}$ é uma raiz de $\mathfrak{h}$ então, devido a expressão 2.4 , temos

$$
H_{\alpha_{i j}}=\frac{1}{2 n}\left(E_{i i}-E_{j j}\right) .
$$

Com isso, dadas duas raízes quaisquer de $\mathfrak{h}$, temos os seus valores da forma de Cartan-Killing

$$
\begin{aligned}
& \left\langle\alpha_{i j}, \alpha_{r s}\right\rangle=\left\langle\frac{1}{2 n}\left(E_{i i}-E_{j j}\right), \frac{1}{2 n}\left(E_{r r}-E_{s s}\right)\right\rangle= \\
& \quad \frac{1}{4 n}\left(\left\langle E_{i i}, E_{r r}\right\rangle-\left\langle E_{i i}, E_{s s}\right\rangle-\left\langle E_{j j}, E_{r r}\right\rangle+\left\langle E_{j j}, E_{s s}\right\rangle\right)=\frac{1}{2 n}\left(\delta_{i r}-\delta_{i s}-\delta_{j r}+\delta_{j s}\right) .
\end{aligned}
$$

Onde $\delta_{i j}=1$ se $i=j$ e 0 caso contrário. Logo, o número de Killing de duas raízes é

$$
\frac{2\left\langle\alpha_{i j}, \alpha_{r s}\right\rangle}{\left\langle\alpha_{i j}, \alpha_{i j}\right\rangle}=\delta_{i r}-\delta_{i s}-\delta_{j r}+\delta_{j s} .
$$

Para a $\alpha_{i j}$-sequência iniciada em $\alpha_{r s}$, existem três possibilidades:

1. $\{i, j\} \cap\{r, s\}=\emptyset$. Nesse caso a sequência consiste apenas de $\alpha_{r s}$, pois $\alpha_{r s}+\alpha_{i j}$ e $\alpha_{r s}-\alpha_{i j}$ não são raízes, já que esses funcionais não são da forma $\lambda_{i}-\lambda_{j}$. 
2. $\{i, j\} \cap\{r, s\}$ tem apenas um elemento. Assim

$$
\frac{2\left\langle\alpha_{i j}, \alpha_{r s}\right\rangle}{\left\langle\alpha_{i j}, \alpha_{i j}\right\}}= \pm 1
$$

e a sequência é formada por $\alpha_{r s}$ e $\alpha_{r s}+\alpha_{i j}$ ou $\alpha_{r s}-\alpha_{i j}$.

3. $\{i, j\}=\{r, s\}$. Então $\alpha_{i j}= \pm \alpha_{r s}$, e assim a sequência é formada por $\pm \alpha_{i j}$ e 0 .

Das raízes $\alpha_{i j}$ podemos escolher o conjunto

$$
\Sigma=\left\{\alpha_{12}, \alpha_{23}, \ldots, \alpha_{n-1, n}\right\}
$$

como o sistema simples de raízes, já que para $i<j$,

$$
\alpha_{i j}=\alpha_{i, i+1}+\ldots+\alpha_{j-1, j} .
$$

Portanto, como $\alpha_{i j}=-\alpha_{j i}$, todas as raízes podem ser escritas como combinação linear com coeficientes inteiros todos positivos ou todos negativos. O número de elementos de $\Sigma$ coincide com a dimensão de $\mathfrak{h}$ e $\Sigma$ gera $\mathfrak{h}_{\mathbb{Q}}^{*}$. A equação 2.5 nos mostra também que as raízes de altura $h$ são $\alpha_{i, i+h} \operatorname{com} i$ variando entre 1 e $n-h$.

Tomando os números de Killing associados a esse sistema simples de raízes temos a matriz de Cartan associada, que é da forma

$$
\left(\begin{array}{ccccc}
2 & -1 & & 0 & \\
-1 & 2 & & & \\
& & \ddots & & \\
& & & 2 & -1 \\
& 0 & & -1 & 2
\end{array}\right) .
$$

De fato, $\left\langle\alpha_{i, i+1}, \alpha_{i+1, i+2}\right\rangle=-1$ e os outros números de Killing se anulam.

Finalmente, o diagrama de Dynkin definido pela matriz de Cartan de $\operatorname{sl}(n)$ é

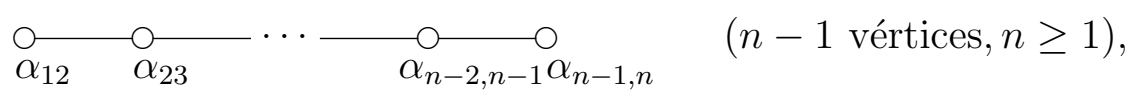




\section{Capítulo 3}

\section{Diagramas de Dynkin}

No capítulo anterior mostramos a correspondência entre as álgebras se Lie semisimples e os diagramas de Dynkin. Neste capítulo o objetivo será encontrar todos os diagramas de Dynkin possíveis, e assim classificar as álgebras semi-simples de dimensão finita sobre corpos algebricamente fechados por esses diagramas.

Vimos, no capítulo anterior, que os diagramas são construídos a partir de um sistema simples de raízes, que é uma base de um espaço vetorial racional com produto interno. Agora iremos esquecer por um momento a sua relação com as álgebras de Lie e iremos analisar apenas a geometria dos elementos de uma base desse tipo, como os ângulos entre os elementos e seus comprimentos relativos.

Observe que para encontrar e classificar os diagramas é suficiente encontrar suas partes conexas, ou seja, as partes onde duas raízes são conectadas por um segmento ou mais. Um diagrama qualquer é sempre uma união disjunta de diagramas conexos. Assim, iremos nos ater ao estudo de quando duas raízes são conectadas, ou seja, quando seu produto interno não se anula (e portanto seu número de Killing não se anula).

\subsection{Classificação dos diagramas}

Primeiramente analisaremos apenas os ângulos entre os elementos da base (sistema simples de raízes), sem nos preocuparmos com os comprimentos desses elementos. Portanto, os diagramas associados a essas bases não terão orientação. Para isso vamos tomar uma base de $\mathbb{Q}^{l}$ normalizada, e desse modo encontrarmos os possíveis diagramas não orientados, verificando apenas o número de arestas que há entre os elementos. Assim, seja

$$
\left\{u_{1}, \ldots, u_{l}\right\}
$$

base tal que $\left|u_{i}\right|=1$ para $i=1, \ldots, l$ e onde os únicos possíveis ângulos entre os elementos são $90^{\circ}, 120^{\circ}, 135^{\circ}$ ou $150^{\circ}$. Os possíveis valores de $\left\langle u_{i}, u_{j}\right\rangle$ são $0,-\frac{1}{2},-\frac{\sqrt{2}}{2},-\frac{\sqrt{3}}{2}$. Fixaremos essa base em todos os lemas dessa seção, exceto quando mencionado o contrário. Serão alguns lemas que nos indicarão os possíveis diagramas associados a essa base. 
Lema 3.1.1. Dado um diagrama, ao retirar alguns vértices juntamente com as arestas incidentes a esses vértices, o que se obtém ainda é um diagrama.

Demonstração: De fato, ao retirar vértices de um diagrama estamos na verdade retirando elementos da base. Sejam $\left\{u_{i_{1}}, \ldots, u_{i_{k}}\right\}$ os elementos que foram retirados. Os elementos que restarem da base, digamos $\left\{u_{j_{1}}, \ldots, u_{j_{m}}\right\}$, formam um conjunto L.I. que está associado a um diagrama. Este diagrama é justamente o diagrama original, a menos dos vértices $\left\{u_{i_{1}}, \ldots, u_{i_{k}}\right\}$ e suas arestas incidentes.

Lema 3.1.2. Num diagrama de l vértices, a quantidade de pares conectados é menor que $l$.

Demonstração: Um par de elementos $\left(u_{i}, u_{j}\right)$ é conectado se $\left\langle u_{i}, u_{j}\right\rangle<0$. Tome $u=u_{1}+\ldots+u_{l}$. Assim $u \neq 0 \mathrm{e}$

$$
\begin{aligned}
0<|u|^{2} & =\left\langle\sum_{i} u_{i}, \sum_{j} u_{j}\right\rangle \\
& =\sum_{i}\left|u_{i}\right|^{2}+2 \sum_{i<j}\left\langle u_{i}, u_{j}\right\rangle \\
& =l+2 \sum_{i<j}\left\langle u_{i}, u_{j}\right\rangle .
\end{aligned}
$$

Portanto,

$$
\sum_{i<j}-2\left\langle u_{i}, u_{j}\right\rangle<l
$$

Os possíveis valores de $-2\left\langle u_{i}, u_{j}\right\rangle$ são $1, \sqrt{2} e \sqrt{3} \geq 1$, se não for zero. Logo, se supormos por absurdo que existem mais do que $l$ pares $\left(u_{i}, u_{j}\right)$ tais que $\left\langle u_{i}, u_{j}\right\rangle \neq 0$, então

$$
l \leq \sum_{i<j}-2\left\langle u_{i}, u_{j}\right\rangle<l
$$

Portanto a quantidade de pares não ortogonais é menor do que $l$.

Definição 3.1.3. Um ciclo em um diagrama é um grafo da forma

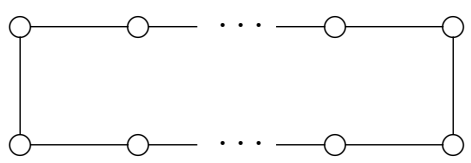


Lema 3.1.4. Um diagrama não contém ciclos.

Demonstração: Um ciclo em um diagrama é um diagrama pelo lema 3.1.1, pois se retirarmos todos os vértices que não estão no ciclo, este ainda será um diagrama. Mas esse diagrama terá $l$ vértices e $l$ arestas, o que não pode ocorrer pelo lema 3.1.2.

Lema 3.1.5. A quantidade de arestas incidentes em um vértice é menor ou igual a 3.

Demonstração: Sejam $u$ um vértice e $v_{1}, \ldots, v_{k}$ os vértices ligados a $u$. O número de arestas entre $v_{i}$ e $u$ é

$$
\frac{2\left\langle v_{i}, u\right\rangle}{\left\langle v_{i}, v_{i}\right\rangle} \frac{2\left\langle v_{i}, u\right\rangle}{\langle u, u\rangle}=4\left\langle v_{i}, u\right\rangle^{2},
$$

pois a base é normalizada. Assim, a quantidade total de arestas incidindo em $u$ é

$$
\sum_{i=1}^{k} 4\left\langle v_{i}, u\right\rangle^{2} .
$$

Sejam $U$ e $V$ os subespaços gerados por $\left\{u, v_{1}, \ldots, v_{k}\right\}$ e $\left\{v_{1}, \ldots, v_{k}\right\}$, respectivamente. O complementar ortogonal a $V$ em $U$ é um subespaço de dimenção 1 . Seja $w$ o vetor de norma 1, que gera esse espaço. Assim o conjunto $\left\{w, v_{1}, \ldots, v_{k}\right\}$ é uma base ortonormal de $U$. De fato, $\left\langle v_{i}, w\right\rangle=0$, para todo $v_{i}$ por construção. $\left\langle v_{i}, v_{j}\right\rangle=0$ pois caso contrário $\operatorname{algum} v_{i}$ seria ligado a $v_{j}$, e haveria um ciclo no diagrama ligado a $u$. Todos esses vetores têm norma um. Portanto,

$$
\begin{gathered}
u=\langle u, w\rangle w+\left\langle u, v_{1}\right\rangle v_{1}+\ldots+\left\langle u, v_{k}\right\rangle v_{k} \Rightarrow \\
|u|^{2}=\langle u, w\rangle^{2}+\left\langle u, v_{1}\right\rangle^{2}+\ldots+\left\langle u, v_{k}\right\rangle^{2}=1
\end{gathered}
$$

concluindo que

$$
\left\langle u, v_{1}\right\rangle^{2}+\ldots+\left\langle u, v_{k}\right\rangle^{2}<1
$$

pois $\langle u, w\rangle \neq 0$, pelo fato que $u \notin V$. Portanto

$$
\sum_{i=1}^{k} 4\left\langle v_{i}, u\right\rangle^{2}<4
$$

Esse último lema mostra que não podemos ter diagramas do tipo

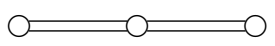

já que há quatro arestas incidindo em um mesmo vértice. O lema nos dá a primeira classificação de diagramas, pois o único diagrama possível com três arestas é 
$G_{2}:$

Para o próximo lema será utilizado o termo cadeia simples para designar um diagrama ou um pedaço do diagrama da forma

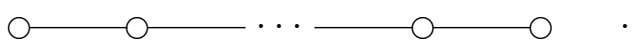

Lema 3.1.6. Suponha que um diagrama contém uma cadeia simples. Então, contraindo a cadeia simples a um vértice e mantendo a esse vértice as ligações com o diagrama restante, o que se obtém ainda é um diagrama.

Um exemplo dessa contração é

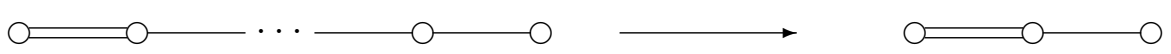

Demonstração: Sejam $\left\{v_{1}, \ldots, v_{k}\right\}$ os vértices da cadeia simples do diagrama, e $\left\{u_{1}, \ldots, u_{r}\right\}$ os vértices restantes. Tome $v=v_{1}+\ldots+v_{k}$. Mostraremos que o diagrama da base $\left\{v, u_{1}, \ldots, u_{r}\right\}$ é o diagrama obtido por contração da cadeia simples. De fato,

$$
\langle v, v\rangle=\sum_{i=1}^{k}\left|v_{i}\right|^{2}+2 \sum_{i<j}\left\langle v_{i}, v_{j}\right\rangle=k+2 \sum_{i<j}\left\langle v_{i}, v_{j}\right\rangle .
$$

Como a cadeia dos $\left\{v_{1}, \ldots, v_{k}\right\}$ é simples, $2\left\langle v_{i}, v_{j}\right\rangle=-1$, se $j=i+1$ e vale zero nos restantes. Logo

$$
|v|^{2}=k+\sum_{i=1}^{k-1} 2\left\langle v_{i}, v_{i+1}\right\rangle=k-(k-1)=1 .
$$

Isso mostra que $v$ tem norma um. Agora tomando um $u_{i}$ arbitrário, temos

$$
\left\langle u_{i}, v\right\rangle=\left\langle u_{i}, v_{j}\right\rangle
$$

para algum $j$. De fato, $u_{i}$ só pode ter conexão com um $v_{j}$, pois se tivesse conexão com mais de um $v_{j}$, haveriam ciclos no diagrama. Portanto $\left\{v, u_{1}, \ldots, u_{r}\right\}$ é um conjunto L.I. formado por vetores normalizados e os ângulos entre seus elementos definem um diagrama dado justamente por $\left\langle u_{i}, v\right\rangle=\left\langle u_{i}, v_{j}\right\rangle$. 
Proposição 3.1.7. Os únicos diagramas conexos (não-orientados) são:

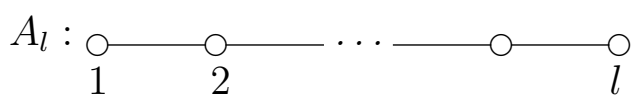

$G_{2}: \bigcirc \equiv$
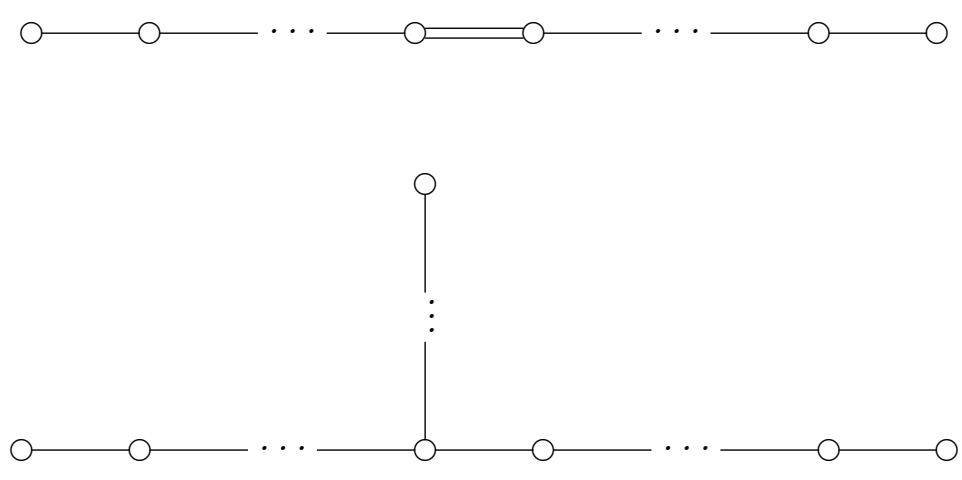

Demonstração: O diagrama $A_{l}$ é o único que não apresenta bifurcações ou ligações múltiplas entre os vértices. O segundo diagrama $G_{2}$ é o único diagrama que apresenta ligações triplas pelo lema 3.1.5. Agora se um diagrama apresenta bifurcações ou ligações duplas, então a partir das extremidades dessas bifurcações ou ligações duplas há uma cadeia simples. Essa cadeia simples não pode ter, em sua outra extremidade, mais bifurcações ou ligações duplas, pois assim, pelo lema 3.1.6, faríamos contrações do tipo
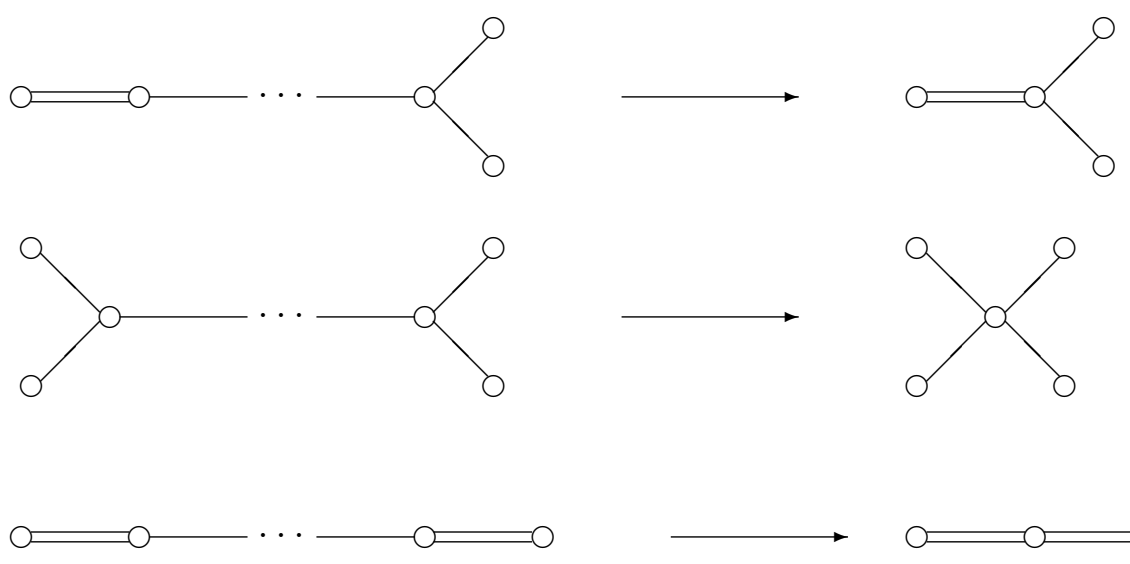

Esses diagramas dariam origem a diagramas que tem vértices com quatro arestas, o que não pode ocorrer.

Agora faremos a análise dos possíveis diagramas que tem ligações duplas ou bifurcações. 
Proposição 3.1.8. Os possíveis diagramas que contém ligações duplas são

$B C_{l}$ :

$F_{4}:$
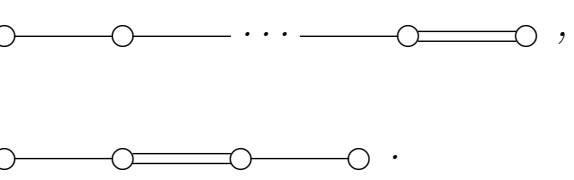

Demonstração: Existem inteiros $p, q \geq 1$ tais que o diagrama é da forma

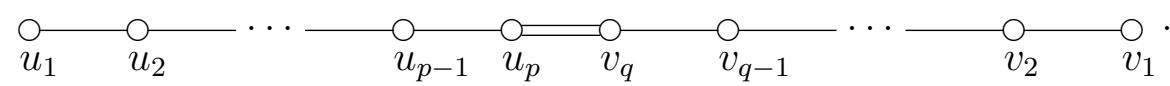

Será mostrado quais são as restrições aos possíveis valores de $p$ e $q$. Tome

$$
u=\sum_{i=1}^{p} i u_{i} \quad e \quad v=\sum_{i=1}^{q} i v_{i} .
$$

Aplicaremos a desigualdade de Cauchy-Schwartz a esses dois vetores. Temos:

$$
\begin{aligned}
\langle u, u\rangle & =\left\langle\sum_{i=1}^{p} i u_{i}, \sum_{j=1}^{p} j u_{j}\right\rangle=\sum_{i=1}^{p} i^{2}\left|u_{i}\right|^{2}+2 \sum_{i<j}\left\langle i u_{i}, j u_{j}\right\rangle \\
& =\sum_{i=1}^{p} i^{2}-\sum_{i=1}^{p-1} i(i+1)=\sum_{i=1}^{p-1} i^{2}+p^{2}-\left(\sum_{i=1}^{p-1} i^{2}+\sum_{i=1}^{p-1} i\right) \\
& =p^{2}-\sum_{i=1}^{p-1} i
\end{aligned}
$$

pois $\left|u_{i}\right|^{2}=1$ e $2\left\langle u_{i}, u_{i+1}\right\rangle=-1$. Analogamente,

$$
|v|^{2}=\frac{q(q+1)}{2}
$$

Temos também

$$
\langle u, v\rangle=\left\langle\sum_{i=1}^{p} i u_{i}, \sum_{i=1}^{q} j v_{j}\right\rangle=\left\langle p u_{p}, q v_{q}\right\rangle=p q\left\langle u_{p}, v_{q}\right\rangle
$$

pois $u_{i}$ é ortogonal a $v_{j}$ se $i<p$ ou $j<q$. Como a ligação entre $u_{p}$ e $v_{q}$ é dupla, $\left\langle u_{p}, v_{q}\right\rangle^{2}=\frac{1}{2}$, e portanto

$$
\langle u, v\rangle^{2}=p^{2} q^{2}\left\langle u_{p}, v_{q}\right\rangle^{2}=\frac{p^{2} q^{2}}{2} .
$$

Agora aplicando a desigualdade de Cauchy-Schwartz, temos

$$
\langle u, v\rangle^{2}<|u|^{2}|v|^{2}
$$


Portanto

$$
\begin{gathered}
\frac{p^{2} q^{2}}{2}<\frac{p(p+1)}{2} \frac{q(q+1)}{2} \Rightarrow \\
\frac{p q}{2}<\frac{p q+p+q+1}{4} \Rightarrow \\
p q-p-q+1<2 .
\end{gathered}
$$

Logo

$$
(p-1)(q-1)<2
$$

Temos dois casos a analisar:

a) $q=1$. Então, não existe restrição a $p$. Origina-se o diagrama $B C_{l}$.

b) $q=2$ Então $p-1<2$ e $p=1$ ou $p=2$. O caso $p=2$ origina o diagrama $F_{4}$, e o caso $p=1$ é o diagrama $B C_{3}$.

Se $q \geq 3$ então $p=1$, o que é o mesmo que o caso a) invertendo os papéis de $p$ e $q$. Portanto todos os casos onde há ligação dupla foram determinados, demonstrando a proposição.

Proposição 3.1.9. Os possíveis diagramas que admitem bifurcação são

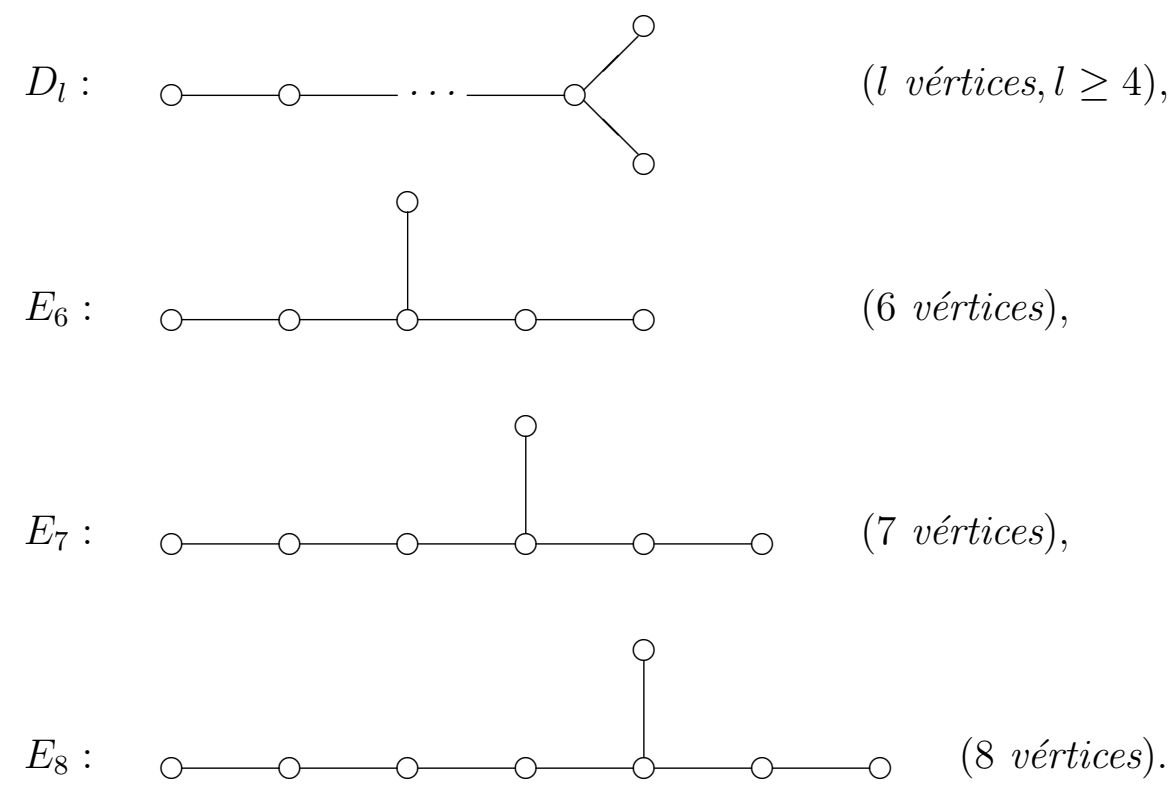

Demonstração: Como na proposição anterior, sejam inteiros $p, q, r>1$, tais que o diagrama é da forma 


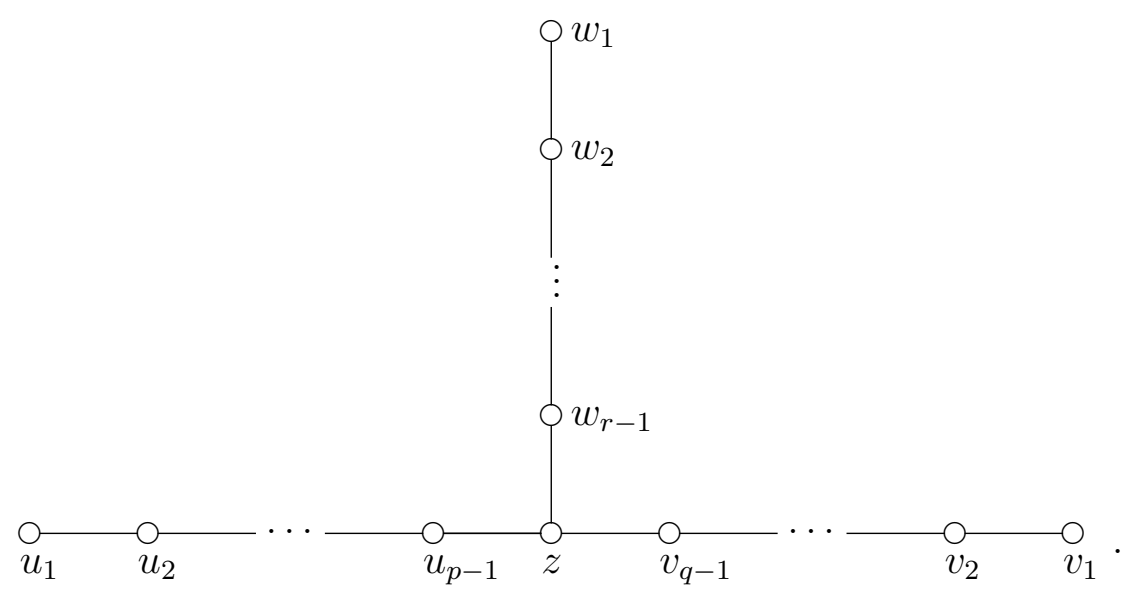

Definimos

$$
u=\sum_{i=1}^{p-1} i u_{i}, \quad v=\sum_{i=1}^{q-1} i v_{i}, \quad w=\sum_{i=1}^{r-1} i w_{i}
$$

Logo

Analogamente,

$$
\begin{aligned}
\langle u, u\rangle & =\left\langle\sum_{i=1}^{p-1} i u_{i}, \sum_{j=1}^{p-1} j u_{j}\right\rangle=\sum_{i=1}^{p-1} i^{2}\left|u_{i}\right|^{2}+2 \sum_{i<j}\left\langle i u_{i}, j u_{j}\right\rangle \\
& =\sum_{i=1}^{p-1} i^{2}-\sum_{i=1}^{p-1} i(i-1)=\sum_{i=1}^{p-1} i^{2}-\sum_{i=1}^{p-1} i^{2}+\sum_{i=1}^{p-1} i \\
& =\frac{p(p-1)}{2} .
\end{aligned}
$$

$$
|v|^{2}=\frac{q(q-1)}{2} \quad \text { e }|w|^{2}=\frac{r(r-1)}{2} .
$$

Seja $V$ o subespaço vetorial gerado por $\{u, v, w\}$ e defina $\theta_{1}, \theta_{2}, \theta_{3}$ os ângulos formados entre $z$ e $u, v$ e $w$, respectivamente. Seja $\operatorname{pr}_{V}(z)$ a projeção do vetor $z$ no espaço $V$. A norma de $\operatorname{pr}_{V}(z)$ é menor que a norma de $z(|z|=1)$ pois $z$ não pertence a $V$. Por outro lado, sabemos que

$$
\left|p r_{V}(z)\right|=\sqrt{\cos ^{2} \theta_{1}+\cos ^{2} \theta_{2}+\cos ^{2} \theta_{3}} .
$$

Logo

$$
\cos ^{2} \theta_{1}+\cos ^{2} \theta_{2}+\cos ^{2} \theta_{3}<1 .
$$

Agora vamos encontrar os valores de $\cos ^{2} \theta_{1}, \cos ^{2} \theta_{2}$ e $\cos ^{2} \theta_{3}$ em função de $p, q$ e $r$. Temos

$$
\langle u, z\rangle=\left\langle\sum_{i=1}^{p-1} i u_{i}, z\right\rangle=\left\langle(p-1) u_{p-1}, z\right\rangle=(p-1)\left(-\frac{1}{2}\right)=\frac{1-p}{2} .
$$

Logo

$$
\cos ^{2} \theta_{1}=\frac{\langle u, z\rangle^{2}}{|u|^{2}|z|^{2}}=\frac{\frac{(1-p)^{2}}{4}}{\frac{p(p-1)}{2}}=\frac{p-1}{2 p}=\frac{1}{2}\left(1-\frac{1}{p}\right) .
$$


Analogamente

$$
\cos ^{2} \theta_{2}==\frac{1}{2}\left(1-\frac{1}{q}\right) \quad \text { e } \cos ^{2} \theta_{3}==\frac{1}{2}\left(1-\frac{1}{r}\right)
$$

Por 3.1,

$$
\begin{gathered}
\frac{1}{2}\left(\left(1-\frac{1}{p}\right)+\left(1-\frac{1}{q}\right)+\left(1-\frac{1}{r}\right)\right)<1 \Rightarrow \\
3-\left(\frac{1}{p}\right)-\left(\frac{1}{q}\right)-\left(\frac{1}{r}\right)<2 \Rightarrow \\
\frac{1}{p}+\frac{1}{q}+\frac{1}{r}>1 .
\end{gathered}
$$

Vamos analisar os casos. Sem perda de generalidade podemos supor $p \geq q \geq r>1$.

1. $r=2$

a) $q=2$. Então $\frac{1}{p}>0$, portanto $p$ pode ser qualquer inteiro maior que 2 , o que origina o diagrama $D_{l}$.

b) $q=3$. Então $\frac{1}{p}+\frac{1}{3}+\frac{1}{2}>1$, $\operatorname{logo} p<6$. Portanto $p=3,4$ ou 5 . Esses casos originam os diagramas $E_{6}, E_{7}$ e $E_{8}$, respectivamente.

c) $q \geq 4$. Então $\frac{1}{p}+\frac{3}{4} \geq \frac{1}{q}+\frac{1}{q}+\frac{1}{2}>1, \operatorname{logo} p<4$. Isso é uma contradição pois supomos que $p \geq q$.

2. $r \geq 3$. Como $q \geq r$, temos

$$
1<\frac{1}{p}+\frac{1}{q}+\frac{1}{r} \leq \frac{1}{p}+\frac{2}{3}
$$

e portanto $p<3$. Mas isso é uma contradição pois supomos que $p \geq q$. Concluímos que não há diagramas com $r \geq 3$.

Esses casos cobrem todos os casos possíveis.

Com esta proposição esgotamos todos os casos de diagramas que provém de bases normalizadas, ou seja, não dirigidas. Agora, para estudarmos o caso onde há direção, basta checarmos os casos onde há ligações duplas e triplas, que são os diagramas $G_{2}, F_{4}$ e $B C_{l}$. Como $G_{2}$ e $F_{4}$ são simétricos em relação a ligação dupla, não há diferença em qual direção fixar. Agora, para o diagrama $B C_{l}$, há duas direções possíveis. Por isso o dividimos em dois diagramas. Vamos concluir com o teorema que deixará tudo explícito.

Teorema 3.1.10. Os diagramas de Dynkin conexos possíveis são:

$A_{l}:$

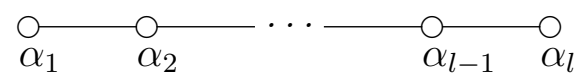

$B_{l}:$

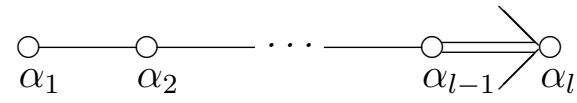

(l vértices, $l \geq 1)$,

( $l$ vértices, $l \geq 2$ ), 
$C_{l}:$

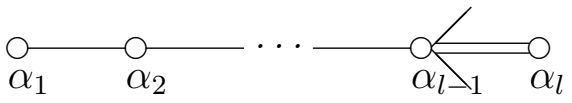

(l vértices, $l \geq 3$ ),

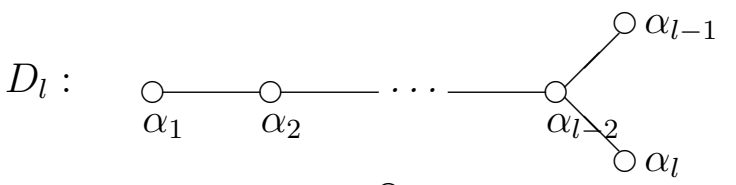

(l vértices, $l \geq 4)$,
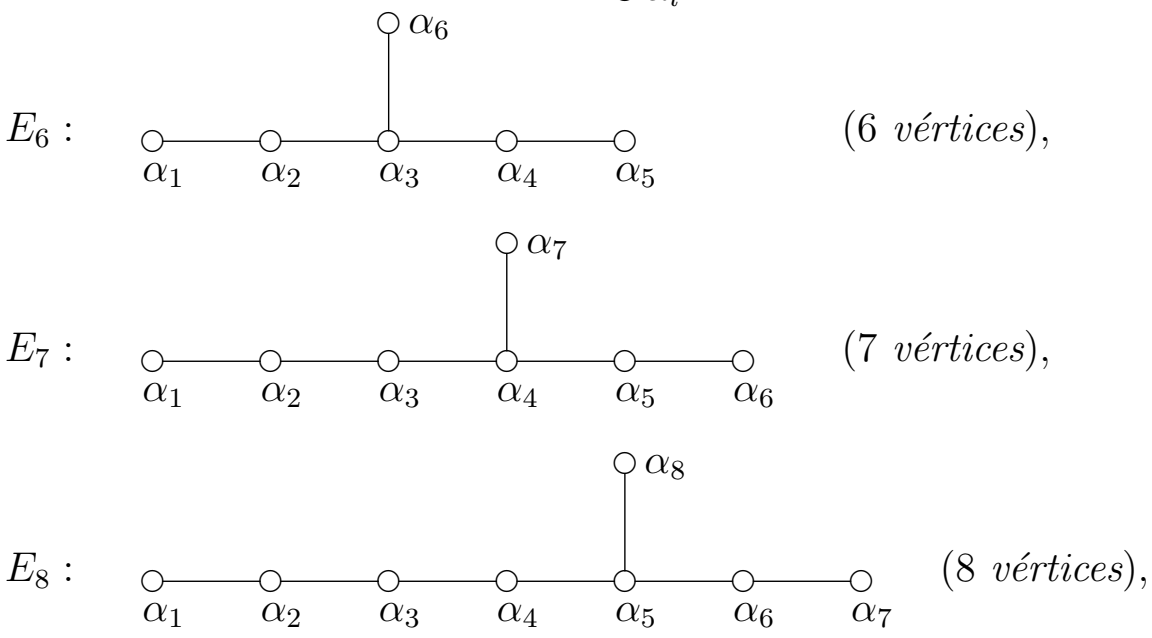

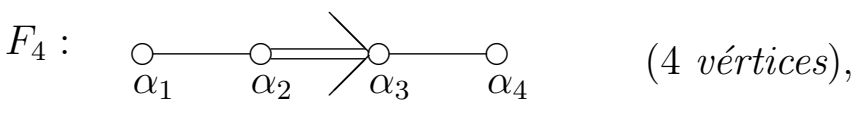

$G_{2}: \quad \varlimsup_{\alpha_{1}} \overbrace{\alpha_{2}} \quad$ (2 vértices).

A demonstração desse teorema segue de todas as proposições feitas durante este capítulo. Mas ainda está incompleta, visto que o teorema diz que esses dão exatamente, os diagramas possíveis. Portanto precisamos mostrar que realmente esses diagramas são diagramas dados por bases de $\mathbb{Q}^{l}$. No próximo capítulo (para o caso das álgebras clássicas) será mostrado que cada uma dessas bases corresponde a um sistema simples de raízes associado a uma subálgebra de Cartan de uma álgebra semi-simples de dimensão finita.

\subsection{Realizações dos diagramas}

Em todos os 7 casos, sejam $\left\{e_{1}, e_{2}, \ldots, e_{l}\right\}$ a base canônica de $\mathbb{Q}^{l}$. Consideramos $\mathbb{Q}^{l}$ $\operatorname{com}\langle\cdot, \cdot\rangle$ o produto interno canônico.

1) $A_{l}: \operatorname{Em} \mathbb{Q}^{l+1}$, seja $E_{l}$ o subespaço

$$
E_{l}=\left\{\left(x_{1}, \ldots, x_{l+1}\right): x_{1}+\ldots+x_{l+1}=0\right\} .
$$

Assim o conjunto

$$
\Sigma_{l}=\left\{e_{1}-e_{2}, e_{2}-e_{3}, \ldots, e_{l}-e_{l+1}\right\}
$$


é uma base de $E_{l}$. Todos os elementos tem o mesmo comprimento e o número de Killing de um par de elementos $\left(e_{i}-e_{i+1}, e_{i+1}-e_{i+2}\right)$ é -1 para todo $i=1, \ldots, l-1$.

2) $B_{l}: \operatorname{Em} \mathbb{Q}^{l}$, seja

$$
\Sigma_{l}=\left\{e_{1}-e_{2}, e_{2}-e_{3}, \ldots, e_{l-1}-e_{l}, e_{l}\right\}
$$

Então $\Sigma_{l}$ é uma base que nos $l-1$ primeiros elementos seguem o mesmo padrão da base do diagrama $A_{l-1}$. Mas o número de Killing entre $e_{l-1}-e_{l}$ e $e_{l}$, é

$$
\frac{2\left\langle e_{l-1}-e_{l}, e_{l}\right\rangle}{\left\langle e_{l}, e_{l}\right\rangle}=-2 .
$$

Logo essa base realiza o diagrama $B_{l}$, para $l \geq 2$.

3) $C_{l}$ : Da mesma forma que no anterior, verifica-se que a base de $\mathbb{Q}^{l}$

$$
\Sigma_{l}=\left\{e_{1}-e_{2}, e_{2}-e_{3}, \ldots, e_{l-1}-e_{l}, 2 e_{l}\right\}
$$

É uma base que realiza o diagrama $C_{l}$, para $l \geq 3$.

4) $D_{l}:$ A base de $\mathbb{Q}^{l}$

$$
\Sigma_{l}=\left\{e_{1}-e_{2}, e_{2}-e_{3}, \ldots, e_{l-2}-e_{l-1}, e_{l-1}-e_{l}, e_{l-1}+e_{l}\right\}
$$

é a que realiza o diagrama $D_{l}$, para $l \geq 4$. O vértice em que ocorre a bifurcação é $e_{l-2}-e_{l-1}$.

5) $E_{6}, E_{7}$ e $E_{8}$ : Primeiro faremos a realizalção do diagrama $E_{8}$. Tome em $\mathbb{Q}^{9}$ o subespaço

$$
\mathbb{E}_{8}=\left\{\left(x_{1}, \ldots, x_{9}\right) \in \mathbb{Q}^{9}: x_{1}+\ldots+x_{9}=0\right\}
$$

e nesse subespaço tome a base

$$
\Sigma_{8}=\left\{e_{2}-e_{3}, e_{3}-e_{4}, \ldots, e_{7}-e_{8}, e_{8}-e_{9}, v\right\},
$$

onde $v$ é a projeção ortogonal do vetor $-\left(e_{2}+e_{3}+e_{4}\right)$ sobre $\mathbb{E}_{8}$, que explicitamente é

$$
v=-\frac{2}{3}\left(e_{2}+e_{3}+e_{4}\right)+\frac{1}{3}\left(e_{1}+e_{5}+e_{6}+e_{7}+e_{8}+e_{9}\right) .
$$

O diagrama começando da esquerda para a direita tem o primeiro vértice $\left(e_{8}-e_{9}\right)$. A bifurcação ocorre no vértice $\left(e_{4}-e_{5}\right)$ que se liga a $v,\left(e_{3}-e_{4}\right)$ e $\left(e_{5}-e_{6}\right)$.

Os diagramas $E_{6}$ e $E_{7}$ são subdiagramas de $E_{8}$, logo são realizados retirando os primeiros vértices de $E_{8}$, que são $\left(e_{8}-e_{9}\right)$ e $\left(e_{7}-e_{8}\right)$.

6) $F_{4}$ : Tome a base de $\mathbb{Q}^{4}$ 


$$
\Sigma_{4}=\left\{e_{1}-e_{2}, e_{2}-e_{3}, e_{2}, \frac{1}{2}\left(-e_{1}-e_{2}-e_{3}+e_{4}\right)\right\} .
$$

Aqui o comprimento dos maiores elementos é $\sqrt{2}$ e dos menores 1 , e a ligação dupla ocorre entre $e_{2}-e_{3}$ e $e_{3}$.

7) $G_{2}$ : Tome em $\mathbb{Q}^{3}$ o subespaço de dimensão 2 gerado pelo par de vetores

$$
\left(0, \frac{1}{6},-\frac{1}{6}\right) \text { e }\left(-\frac{1}{18},-\frac{1}{18}, \frac{2}{18}\right) .
$$

Assim essa base de vetores gera o diagrama $G_{2}$, pois formam um ângulo de $150^{\circ}$. 


\section{Capítulo 4}

\section{Álgebras Semi-simples}

No capítulo anterior foi estabelecida a relação entre as álgebras semi-simples, os diagramas de Dynkin e os possíveis diagramas que podem existir. Esse capítulo terá como objetivo mostrar que os diagramas de Dynkin estão biunivocamente relacionados com as álgebras semi-simples (a menos de isomorfismo).

\section{1 Álgebras Isomorfas e seus Diagramas de Dynkin}

A primeira pergunta que iremos fazer é se dois sistemas simples de raízes de duas subálgebras de Cartan, da álgebra semi-simples $\mathfrak{g}$, têm o mesmo diagrama de Dynkin.

Proposição 4.1.1. Sejam $\mathfrak{h}_{1}$ e $\mathfrak{h}_{2}$ subálgebras de Cartan de uma álgebra de Lie semisimples $\mathfrak{g}$. Sejam $\Sigma_{1}$ e $\Sigma_{2}$ dois sistemas simples de raízes associados às subálgebras $\mathfrak{h}_{1}$ $e \mathfrak{h}_{2}$ respectivamente. Então os diagramas associados a $\Sigma_{1}$ e $\Sigma_{2}$ coincidem.

Demonstração: Pelos resultados apresentados no capítulo 1, como o corpo é algebricamente fechado, existe um automorfismo de $\mathfrak{g}, \phi$, tal que $\phi\left(\mathfrak{h}_{1}\right)=\mathfrak{h}_{2}$. Sejam $\Pi_{1}$ e $\Pi_{2}$ o conjunto de raízes de $\mathfrak{h}_{1}$ e $\mathfrak{h}_{2}$ respectivamente. Tome $\alpha \in \Pi_{1}$, então existe $X \neq 0$ tal que

$$
\operatorname{ad}(H) X=\alpha(H) X, \quad \forall H \in \mathfrak{h}_{1}
$$

Logo

$$
\operatorname{ad}(\phi(H)) \phi(X)=\phi(\operatorname{ad}(H)(X))=\phi(\alpha(H) X)=\alpha(H) \phi(X), \quad \forall H \in \mathfrak{h}_{1} .
$$

Como $\phi$ é automorfismo, para cada $H \in \mathfrak{h}_{1}$ existe um $G \in \mathfrak{h}_{2}$ tal que $\phi^{-1}(G)=H$. Logo

$$
a d(G) \phi(X)=a d\left(\phi\left(\phi^{-1}(G)\right)\right) \phi(X)=\phi\left(\left[\phi^{-1}(G), X\right]\right)=\alpha\left(\phi^{-1}(G)\right) \phi(X),
$$

para todo $G \in \mathfrak{h}_{2}$. Logo $\alpha \circ \phi^{-1}$ é uma raiz de $\mathfrak{h}_{2}$. Considere a aplicação $\phi^{*}$ definida por 


$$
\begin{aligned}
\phi^{*}: \Pi_{1} & \longrightarrow \Pi_{2} \\
\alpha & \longrightarrow \phi^{*}(\alpha)=\alpha \circ \phi^{-1} .
\end{aligned}
$$

Então $\phi^{*}\left(\Pi_{1}\right) \subset \Pi_{2}$. Como $\phi$ é automorfismo e $\Pi_{1}, \Pi_{2}$ finitos, então $\phi^{*}\left(\Pi_{1}\right)=\Pi_{2}$. Se tomarmos $\Sigma_{1} \subset \Pi_{1}$ um sistema simples de raízes, $\phi^{*}\left(\Sigma_{1}\right)$ será uma sistema simples de raízes de $\Pi_{2}$. Como $\phi$ é invariante pela forma de Cartan-Killing, a aplicação $\phi^{*}$ é uma isometria entre as formas de Cartan-Killing em $\mathfrak{h}_{1}^{*}$ e $\mathfrak{h}_{2}^{*}$, pois os inteiros $p$ e $q$ da $\alpha$-sequência iniciada em $\beta$ e da $\phi^{*}(\alpha)$-sequência iniciada em $\phi^{*}(\beta)$ são iguais. Logo os números de Killing entre as raízes e suas imagens por $\phi^{*}$ serão os mesmos. Portanto, os diagramas de $\Sigma_{1}$ e $\phi^{*}\left(\Sigma_{1}\right)$ serão o mesmo.

Proposição 4.1.2. Se $\mathfrak{g}_{1}$ e $\mathfrak{g}_{2}$ são duas álgebras isomorfas, então seus diagramas coincidem.

Demonstração: O procedimento é o mesmo que na proposição 4.1 .1 pois, se $\mathfrak{h}_{1}$ é uma subálgebra de Cartan de $\mathfrak{g}_{1}$ e $\phi$ é o isomorfismo entre as álgebras, então $\phi\left(\mathfrak{h}_{1}\right)$ é uma subálgebra de Cartan de $\mathfrak{g}_{2}$. Além disso, $\phi^{*}$ é uma isometria entre as formas de Cartan-Killing em $\mathfrak{h}_{1}^{*}$ e $\phi\left(\mathfrak{h}_{1}\right)^{*}$. Portanto os seus sistemas simples de raízes correspondentes terão o mesmo diagrama.

Proposição 4.1.3. Seja $\mathfrak{g}$ uma álgebra semi-simples e

$$
\mathfrak{g}=\mathfrak{g}_{1} \oplus \mathfrak{g}_{2} \oplus \ldots \oplus \mathfrak{g}_{s}
$$

sua decomposição em componentes simples. Então, o diagrama de $\mathfrak{g}$ se decompõe na união disjunta dos diagramas de $\mathfrak{g}_{1}, \mathfrak{g}_{2}, \ldots, \mathfrak{g}_{s}$, que não estão ligados entre si.

Demonstração: Tome $\mathfrak{h}_{i}$ as subálgebras de Cartan de $\mathfrak{g}_{i}, i=1, \ldots, s$. Sabemos que $\mathfrak{h}_{1} \oplus \ldots \oplus \mathfrak{h}_{s}$ é subálgebra de Cartan de $\mathfrak{g}$. Seja $\Pi_{i}$ o conjunto de raízes de $\mathfrak{h}_{i}$, então $\alpha \in \Pi_{i}$ pode ser extendido a um funcional linear de $\mathfrak{h}$, tomando $\alpha\left(\mathfrak{h}_{j}\right)=0$ se $i \neq j$. Por essas extensões, $\Pi=\Pi_{1} \cup \Pi_{2} \cup \ldots \cup \Pi_{s}$ é o conjunto de raízes de $\mathfrak{h}$ em $\mathfrak{g}$. Temos, por outro lado, que as componentes $\mathfrak{g}_{i}$ são ortogonais em relação a forma de Cartan-Killing. Assim, se $\alpha \in \Pi_{i}$ e $\beta \in \Pi_{j}$, então

$$
\langle\alpha, \beta\rangle=\left\langle H_{\alpha}, H_{\beta}\right\rangle=0
$$

se $i \neq j$. Isso nos diz que as partes correspondentes de $\Pi_{i}$ e $\Pi_{j}$ no diagrama não são ligadas, demonstrando o que queríamos. 
Agora, apenas para o próximo teorema, fixe uma base de $\mathfrak{g}$ que seja união das bases $\operatorname{dos} \mathfrak{g}\left(\alpha_{i}\right) \simeq \operatorname{sl}(2)$. Assim a base é formada por $X_{\alpha_{i}}, Y_{-\alpha_{i}}$ e $H_{\alpha_{i}}$, tal que satifazem as relações:

$$
\begin{aligned}
{\left[H_{\alpha_{i}}, H_{\alpha_{j}}\right] } & =0 \\
{\left[X_{\alpha_{i}}, Y_{-\alpha_{j}}\right] } & =\delta_{i j} H_{\alpha_{i}} \\
{\left[X_{\alpha_{i}}, H_{\alpha_{j}}\right] } & =\frac{2\left\langle\alpha_{i}, \alpha_{j}\right\rangle}{\left\langle\alpha_{j}, \alpha_{j}\right\rangle} X_{\alpha_{i}} \\
{\left[Y_{-\alpha_{i}}, H_{\alpha_{j}}\right] } & =-\frac{2\left\langle\alpha_{i}, \alpha_{j}\right\rangle}{\left\langle\alpha_{j}, \alpha_{j}\right\rangle} Y_{-\alpha_{i}} .
\end{aligned}
$$

A primeira equação vale pois $\mathfrak{h}$ é abeliana. Essa base foi construída na proposição 2.3.1. A notação foi alterada para facilitar a demonstração do teorema: $X_{\alpha}:=X_{\alpha_{i}}$; $Y_{-\alpha}:=Y_{-\alpha_{i}} ; H_{\alpha}^{\prime}:=H_{\alpha_{i}}$. Os detalhes da construção dessa base estão no livro [3].

Teorema 4.1.4. Uma álgebra de Lie $\mathfrak{g}$ é simples se, e somente se, seu diagrama é conexo.

Demonstração: Seja $\mathfrak{h} \subset \mathfrak{g}$ uma subálgebra de Cartan, $\Pi$ as raízes e $\Sigma$ um sistema simples de raízes. Suponha por absurdo que o diagrama não seja conexo, assim $\Sigma$ satisfaz: $\Sigma=\Sigma_{1} \cup \Sigma_{2}$ com $\Sigma_{1} \neq \emptyset$ e $\Sigma_{2} \neq \emptyset$. Tal que $\langle\alpha, \beta\rangle=0$ se $\alpha \in \Sigma_{1}$ e $\beta \in \Sigma_{2}$. Para facilitar a demonstração vamos ordenar $\Sigma$ tal que $\Sigma_{1}=\left\{\alpha_{1}, \ldots, \alpha_{k}\right\}$ e $\Sigma_{2}=\left\{\alpha_{k+1}, \ldots, \alpha_{l}\right\}$. Seja

$$
\mathfrak{g}_{1}=\mathfrak{h}_{1}+\sum_{\alpha \in \Pi_{1}} \mathfrak{g}_{\alpha}
$$

onde $\mathfrak{h}_{1}=\left\{H_{\alpha} \in \mathfrak{g} ; \alpha \in \Pi_{1}\right\}$ e $\Pi_{1}$ é o conjunto gerado por $\Sigma_{1}$ em $\mathfrak{h}^{*}$. Agora se $j \leq k<r$, temos

$$
\left\langle\alpha_{j}, \alpha_{r}\right\rangle=0
$$

Como $\alpha_{j}-\alpha_{r}$ não é raiz, se analisarmos os inteiros $p$ e $q$ da $\alpha_{r}$-sequência iniciada em $\alpha_{j}$, teremos $p=0, \log \mathrm{O}$

$$
0=\frac{2\left\langle\alpha_{j}, \alpha_{r}\right\rangle}{\left\langle\alpha_{j}, \alpha_{j}\right\rangle}=p-q=-q .
$$

Portanto $q=0$ e assim $\alpha_{j}+\alpha_{r}$ também não é raiz. Logo

$$
\begin{gathered}
{\left[X_{\alpha_{j}}, X_{\alpha_{r}}\right] \in \mathfrak{g}_{\alpha_{j}+\alpha_{r}}=\{0\}, j \leq k<r,} \\
{\left[Y_{-\alpha_{j}}, X_{\alpha_{r}}\right] \in \mathfrak{g}_{\alpha_{j}-\alpha_{r}}=\{0\}, j \leq k<r,} \\
{\left[H_{\alpha_{j}}, X_{\alpha_{r}}\right]=\frac{2\left\langle\alpha_{j}, \alpha_{r}\right\rangle}{\left\langle\alpha_{j}, \alpha_{j}\right\rangle} X_{\alpha_{r}}=0, j \leq k<r .}
\end{gathered}
$$

Logo $X_{\alpha_{r}}$ está no normalizador de $\mathfrak{g}_{1}$, se $r>k$. Analogamente vemos que $Y_{\alpha_{r}}$ também está no normalizador de $\mathfrak{g}_{1}$, se $r>k$. Finalmente 


$$
\left[H_{\alpha_{r}}, \mathfrak{g}_{1}\right]=\left[H_{\alpha_{r}}, \mathfrak{h}_{1}\right]+\left[H_{\alpha_{r}}, \sum_{\alpha \in \Pi_{1}} \mathfrak{g}_{\alpha}\right]=0,
$$

pelas relações definidas entre os elementos $X_{\alpha_{i}}, Y_{-\alpha_{i}}$ e $H_{\alpha_{i}}$. Portanto $\mathfrak{g}_{1}$ é um ideal de $\mathfrak{g}$. Absurdo, pois $\mathfrak{g}$ é simples.

Reciprocamente, suponha por absurdo que $\mathfrak{g}$ não é simples. Seja então $\mathfrak{g}=\mathfrak{g}_{1} \oplus \mathfrak{g}_{2}$, tal que os subespaços $\mathfrak{g}_{i}$ são ideais não nulos de $\mathfrak{g}$. Sejam $\alpha$ uma raiz não nula e o respectivo $X_{\alpha} \in \mathfrak{g}_{\alpha}$. Assim $X_{\alpha}=X_{\alpha}^{1}+X_{\alpha}^{2}$, onde $X_{\alpha}^{i} \in \mathfrak{g}_{i}$. Temos $\left[X_{\alpha}, H\right]=\alpha(H) X_{\alpha}$, para todo $H \in \mathfrak{h}$. Logo $\left[X_{\alpha}^{i}, H\right]=\alpha(H) X_{\alpha}^{i}$. Mas como $\mathfrak{g}_{\alpha}$ é de dimensão um, $\mathfrak{g}_{\alpha} \subset \mathfrak{g}_{1}$ ou $\mathfrak{g}_{\alpha} \subset \mathfrak{g}_{2}$. Como $\left[\mathfrak{g}_{1}, \mathfrak{g}_{2}\right]=0$ e $\left[\mathfrak{g}_{\alpha}, \mathfrak{g}_{-\alpha}\right] \neq 0$, temos $\mathfrak{g}_{\alpha}+\mathfrak{g}_{-\alpha} \subset \mathfrak{g}_{1}$ ou $\mathfrak{g}_{\alpha}+\mathfrak{g}_{-\alpha} \subset \mathfrak{g}_{2}$.

Logo podemos ordenar os elementos da base tal que $X_{\alpha_{1}}, Y_{-\alpha_{1}}, \ldots, X_{\alpha_{k}}, Y_{-\alpha_{k}} \in \mathfrak{g}_{1}$ e $X_{\alpha_{k+1}}, Y_{-\alpha_{k+1}}, \ldots, X_{\alpha_{l}}, Y_{-\alpha_{l}} \in \mathfrak{g}_{2}$. Novamente, como $\mathfrak{g}_{1}$ e $\mathfrak{g}_{2}$ são ideais não nulos, temos

$$
0=\left[X_{\alpha_{j}},\left[X_{\alpha_{r}}, Y_{-\alpha_{r}}\right]\right]=\left[X_{\alpha_{j}}, H_{\alpha_{r}}\right]=\frac{2\left\langle\alpha_{r}, \alpha_{j}\right\rangle}{\left\langle\alpha_{r}, \alpha_{r}\right\rangle} X_{\alpha_{j}},
$$

se $j \leq k$ e $r>k$. Logo

$$
\frac{2\left\langle\alpha_{r}, \alpha_{j}\right\rangle}{\left\langle\alpha_{r}, \alpha_{r}\right\rangle}=0
$$

se $j \leq k$ e $r>k$. Portanto o diagrama não é conexo.

Agora iremos nos direcionar para o principal resultado dessa teoria de classificação das álgebras de Lie semi-simples por diagramas de Dynkin: Duas álgebras que têm o mesmo diagrama de Dynkin são isomorfas.

Primeiramente definiremos uma notação, em seguida faremos uma proposição e alguns lemas que nos darão ferramentas para a demonstração desse teorema.

Fixe uma base de $\mathfrak{g}$ formada por uma base de $\mathfrak{h}$ e por $X_{\alpha} \in \mathfrak{g}_{\alpha}$, de tal forma que

$$
\left\langle X_{\alpha}, X_{-\alpha}\right\rangle=1
$$

Tal base existe pelo parte 2. do lema 2.2.6. Esse mesmo lema diz que $\operatorname{dimg}_{\alpha}=1$, e portanto $\mathfrak{g}_{\alpha}=\left\langle X_{\alpha}\right\rangle$. Sejam agora $m_{\alpha, \beta}$ números definidos por

$$
\left[X_{\alpha}, X_{\beta}\right]=m_{\alpha, \beta} X_{\alpha+\beta} .
$$

Assim $m_{\alpha, \beta}=0$ se $\alpha+\beta$ não for raiz. Fica claro, pela anticomutatividade do colchete de Lie que, $m_{\alpha, \beta}=-m_{\beta, \alpha}$.

Proposição 4.1.5. Sejam $\alpha$ e $\beta$ raízes e

$$
\beta-p \alpha, \ldots, \beta, \ldots, \beta+q \alpha
$$

a $\alpha$-sequência iniciada em $\beta$. Então 


$$
\left[X_{-\alpha},\left[X_{\alpha}, X_{\beta}\right]\right]=q(p+1) \frac{\langle\alpha, \alpha\rangle}{2} X_{\beta}
$$

Demonstração: Seja $\mathfrak{g}(\alpha)$ a álgebra isomorfa a $\operatorname{sl}(2)$, ou seja,

$$
\mathfrak{g}(\alpha)=\mathfrak{g}_{\alpha} \oplus\left\langle H_{\alpha}\right\rangle \oplus \mathfrak{g}_{-\alpha}
$$

Como na construção feita na proposição 2.3.1, exitem $X_{\alpha} \in \mathfrak{g}_{\alpha}$ e $Y_{-\alpha} \in \mathfrak{g}_{-\alpha}$ tais que,

$$
\left\langle X_{\alpha}, Y_{-\alpha}\right\rangle=\frac{2}{\langle\alpha, \alpha\rangle}
$$

Pela equação 4.5 temos $Y_{-\alpha}=\frac{2}{\langle\alpha, \alpha\rangle} X_{-\alpha}$. Tome a representação adjunta restrita a $\mathfrak{g}(\alpha)$,

$$
a d: \mathfrak{g}(\alpha) \rightarrow g l(V)
$$

onde $V=\mathfrak{g}_{\beta-p \alpha} \oplus \ldots \oplus \mathfrak{g}_{\beta+q \alpha}$. Essa representação é irredutível pelo que foi feito na demonstração do teorema 2.3.5. Logo existem $\left\{v_{0}, v_{1}, \ldots, v_{p+q}\right\}$ tais que

$$
\begin{gathered}
a d\left(X_{\alpha}\right) v_{i}=\left[X_{\alpha}, v_{i}\right]=i((p+q)-i+1) v_{i-1}, \\
a d\left(Y_{-\alpha}\right) v_{i}=\left[Y_{-\alpha}, v_{i}\right]=v_{i+1}
\end{gathered}
$$

onde $v_{i} \in \mathfrak{g}_{\beta+(q-i) \alpha}$. Logo $v_{q} \in \mathfrak{g}_{\beta}$, e assim

$$
\left[Y_{-\alpha},\left[X_{\alpha}, v_{q}\right]\right]=\left[Y_{-\alpha}, q(p+q-q+1) v_{q-1}\right]=q(p+1)\left[Y_{-\alpha}, v_{q-1}\right]=q(p+1) v_{q} .
$$

Como $X_{\beta}=k v_{q}$ para algum $k \in \mathbb{K}$, a mesma igualdade vale para $X_{\beta}$, logo

$$
\left[Y_{-\alpha},\left[X_{\alpha}, X_{\beta}\right]\right]=q(p+1) X_{\beta}
$$

Portanto

$$
\left[X_{-\alpha},\left[X_{\alpha}, X_{\beta}\right]\right]=\frac{\langle\alpha, \alpha\rangle}{2} q(p+1) X_{\beta}
$$

Lema 4.1.6. Se $\alpha$ e $\beta$ são raízes $e$

$$
\beta-p \alpha, \ldots, \beta+q \alpha
$$

é a $\alpha$-sequência iniciada em $\beta$. Então

$$
m_{\alpha, \beta} m_{-\alpha,-\beta}=-q(p+1) \frac{\langle\alpha, \alpha\rangle}{2} .
$$


Demonstração: Por um lado, temos

$$
\left\langle\left[X_{\alpha}, X_{\beta}\right],\left[X_{-\alpha}, X_{-\beta}\right]\right\rangle=\left\langle m_{\alpha, \beta} X_{\alpha+\beta}, m_{-\alpha,-\beta} X_{-(\alpha+\beta)}\right\rangle=m_{\alpha, \beta} m_{-\alpha,-\beta}
$$

e por outro lado,

$$
\begin{aligned}
& \left\langle\left[X_{\alpha}, X_{\beta}\right],\left[X_{-\alpha}, X_{-\beta}\right]\right\rangle=-\left\langle\left[X_{-\alpha},\left[X_{\alpha}, X_{\beta}\right]\right], X_{-\beta}\right\rangle \\
& =-\left\langle\frac{\langle\alpha, \alpha\rangle}{2} q(p+1) X_{\beta}, X_{-\beta}\right\rangle=-\frac{\langle\alpha, \alpha\rangle}{2} q(p+1) .
\end{aligned}
$$

A primeira igualdade vale pela propriedade da forma de Cartan-Killing e derivações. A segunda igualdade pela proposição anterior.

Lema 4.1.7. Sejam $\alpha, \beta, \gamma \in \Pi$ e suponha que $\alpha+\beta+\gamma=0$. Então

$$
m_{\alpha, \beta}=m_{\beta, \gamma}=m_{\gamma, \alpha} .
$$

Demonstração: Primeiro observemos que $\alpha, \beta$ e $\gamma$ são duas a duas linearmente independentes, pois as únicas raízes que são múltiplos de raízes são ela mesma e sua oposta, o que não ocorre aqui. Logo os seus correspondetes $H_{\alpha}, H_{\beta}$ e $H_{\gamma} \in \mathfrak{h}$ também são linearmente independentes. Agora, aplicando a identidade de Jacobi para $X_{\alpha}, X_{\beta}$ e $X_{\gamma}$, temos

$$
\begin{aligned}
{\left[X_{\alpha},\left[X_{\beta}, X_{\gamma}\right]\right] } & =\left[\left[X_{\alpha}, X_{\beta}\right], X_{\gamma}\right]+\left[X_{\beta},\left[X_{\alpha}, X_{\gamma}\right]\right] \Rightarrow \\
m_{\beta, \gamma}\left[X_{\alpha}, X_{\beta+\gamma}\right] & =m_{\alpha, \beta}\left[X_{\alpha+\beta}, X_{\gamma}\right]+m_{\alpha, \gamma}\left[X_{\beta}, X_{\alpha+\gamma}\right] .
\end{aligned}
$$

Por hipótese temos que $\beta+\gamma=-\alpha$, $\log X_{\beta+\gamma}=X_{-\alpha}$ e portanto

$$
\left[X_{\alpha}, X_{\beta+\gamma}\right]=\left[X_{\alpha}, X_{-\alpha}\right]=H_{\alpha} .
$$

Utilizando a mesma notação e aplicando o mesmo raciocínio para os outros membros da equação 4.6 temos

$$
\begin{gathered}
m_{\beta, \gamma} H_{\alpha}=m_{\alpha, \beta} H_{-\gamma}+m_{\alpha, \gamma} H_{\beta} \Rightarrow \\
m_{\beta, \gamma} H_{\alpha}=m_{\alpha, \beta} H_{\alpha+\beta}+m_{\alpha, \gamma} H_{\beta}=m_{\alpha, \beta}\left(H_{\alpha}+H_{\beta}\right)+m_{\alpha, \gamma} H_{\beta} \Rightarrow \\
\left(m_{\alpha, \beta}-m_{\beta, \gamma}\right) H_{\alpha}+\left(m_{\alpha, \beta}+m_{\alpha, \gamma}\right) H_{\beta}=0 .
\end{gathered}
$$

Como $H_{\alpha}$ e $H_{\beta}$ são linearmente independentes,

$$
m_{\alpha, \beta}=m_{\beta, \gamma}
$$

$\mathrm{e}$

$$
m_{\alpha, \beta}=-m_{\alpha, \gamma}=m_{\gamma, \alpha} .
$$


Lema 4.1.8. Sejam $\alpha, \beta, \gamma$ e $\delta$ raízes e suponha que nenhuma é oposta da outra. Suponha também que

$$
\alpha+\beta+\gamma+\delta=0
$$

Então

$$
m_{\alpha, \beta} m_{\gamma, \delta}+m_{\beta, \gamma} m_{\alpha, \delta}+m_{\gamma, \alpha} m_{\beta, \delta}=0 .
$$

Demonstração: Observe inicialmente que faz sentido a igualdade

$$
\left[X_{\alpha},\left[X_{\beta}, X_{\gamma}\right]\right]=\left[X_{\alpha}, m_{\beta, \gamma} X_{\beta+\gamma}\right]=m_{\beta, \gamma} m_{\alpha, \beta+\gamma} X_{\alpha+\beta+\gamma}=m_{\beta, \gamma} m_{\alpha, \beta+\gamma} X_{-\delta}
$$

mesmo que $\beta+\gamma$ não fosse raiz. De fato, nesse caso $\left[X_{\beta}, X_{\gamma}\right]=0$ e $m_{\beta, \gamma}=0$. Se aplicarmos o lema 4.1 .7 às raízes $\alpha, \beta+\gamma$ e $\delta$, teríamos

$$
m_{\alpha, \beta+\gamma}=m_{\delta, \alpha}
$$

Utilizando as duas equações obtemos

$$
\left[X_{\alpha},\left[X_{\beta}, X_{\gamma}\right]\right]=m_{\alpha, \beta+\gamma} m_{\beta, \gamma} X_{-\delta}=m_{\delta, \alpha} m_{\beta, \gamma} X_{-\delta}=-m_{\alpha, \delta} m_{\beta, \gamma} X_{-\delta} .
$$

Agora, tome a identidade de Jacobi

$$
\begin{gathered}
{\left[X_{\alpha},\left[X_{\beta}, X_{\gamma}\right]\right]=\left[\left[X_{\alpha}, X_{\beta}\right], X_{\gamma}\right]+\left[X_{\beta},\left[X_{\alpha}, X_{\gamma}\right]\right] \Rightarrow} \\
-m_{\alpha, \delta} m_{\beta \gamma} X_{-\delta}=m_{\alpha, \beta}\left[X_{\alpha+\beta}, X_{\gamma}\right]+m_{\alpha, \gamma}\left[X_{\beta}, X_{\alpha+\gamma}\right] \Rightarrow \\
-m_{\alpha, \delta} m_{\beta \gamma} X_{-\delta}=m_{\alpha, \beta} m_{\gamma, \delta} X_{-\delta}+m_{\alpha, \gamma} m_{\delta, \beta} X_{-\delta} .
\end{gathered}
$$

Portanto

$$
\left(m_{\alpha, \beta} m_{\gamma, \delta}+m_{\beta, \gamma} m_{\alpha, \delta}+m_{\gamma, \alpha} m_{\beta, \delta}\right) X_{-\delta}=0
$$

concluindo o lema.

Lema 4.1.9. Sejam $\mathfrak{g}^{1}$ e $\mathfrak{g}^{2}$ álgebras simples e tome subálgebras de Cartan $\mathfrak{h}_{1} \subset \mathfrak{g}^{1} e$ $\mathfrak{h}_{2} \subset \mathfrak{g}^{2}$. Denote por $\Pi_{1}$ e $\Pi_{2}$ os conjuntos de raízes correspondentes. Suponha que exista um isomorfismo $\phi: \mathfrak{h}_{1 \mathbb{Q}}^{*} \rightarrow \mathfrak{h}_{2 \mathbb{Q}}^{*}$ tal que $\phi\left(\Pi_{1}\right)=\Pi_{2}$. Então

$$
\langle\phi(\alpha), \phi(\beta)\rangle=c\langle\alpha, \beta\rangle \text {. }
$$

Demonstração: A imagem por $\phi$ da $\alpha$-sequência iniciada em $\beta$ coincide com a $\phi(\alpha)$ sequência iniciada em $\phi(\beta)$. Logo a fórmula de Killing nos garante que

$$
\frac{\langle\alpha, \beta\rangle}{\langle\beta, \beta\rangle}=\frac{\langle\phi(\alpha), \phi(\beta)\rangle}{\langle\phi(\beta), \phi(\beta)\rangle} .
$$

Portanto 


$$
\langle\alpha, \beta\rangle=\frac{\langle\beta, \beta\rangle}{\langle\phi(\beta), \phi(\beta)\rangle}\langle\phi(\alpha), \phi(\beta)\rangle .
$$

Por outro lado,

$$
\frac{\langle\alpha, \beta\rangle}{\langle\alpha, \alpha\rangle}=\frac{\langle\phi(\alpha), \phi(\beta)\rangle}{\langle\phi(\alpha), \phi(\alpha)\rangle}
$$

$\log 0$

$$
\langle\alpha, \beta\rangle=\frac{\langle\alpha, \alpha\rangle}{\langle\phi(\alpha), \phi(\alpha)\rangle}\langle\phi(\alpha), \phi(\beta)\rangle .
$$

Portanto

$$
\frac{\langle\beta, \beta\rangle}{\langle\phi(\beta), \phi(\beta)\rangle}=\frac{\langle\alpha, \alpha\rangle}{\langle\phi(\alpha), \phi(\alpha)\rangle},
$$

se $\langle\alpha, \beta\rangle \neq 0$. Logo a constante

$$
c=\frac{\langle\beta, \beta\rangle}{\langle\phi(\beta), \phi(\beta)\rangle}=\frac{\langle\alpha, \alpha\rangle}{\langle\phi(\alpha), \phi(\alpha)\rangle}
$$

independe de $\alpha$ ou $\beta$, se $\langle\alpha, \beta\rangle \neq 0$. Portanto a igualdade

$$
\langle\phi(\alpha), \phi(\beta)\rangle=c\langle\alpha, \beta\rangle
$$

vale se $\langle\alpha, \beta\rangle \neq 0$. A igualdade continua valendo se $\langle\alpha, \beta\rangle=0$, pois nesse caso,

$$
\langle\phi(\alpha), \phi(\beta)\rangle=\frac{\langle\phi(\beta), \phi(\beta)\rangle}{\langle\beta, \beta\rangle}\langle\alpha, \beta\rangle=0
$$

Utilizando essas informações sobre as constantes de estrutura de álgebras de Lie e o lema 4.1.9, podemos mostrar o teorema mais importante dessa seção.

Teorema 4.1.10. Suponha que $\mathfrak{g}^{1}$ e $\mathfrak{g}^{2}$ sejam álgebras simples e tome subálgebras de Cartan $\mathfrak{h}_{1} \subset \mathfrak{g}^{1} e \mathfrak{h}_{2} \subset \mathfrak{g}^{2}$. Denote por $\Pi_{1} e \Pi_{2}$ os conjuntos de raízes correspondentes $e$ sejam $\mathfrak{h}_{1 \mathbb{Q}}^{*}$ e $\mathfrak{h}_{2 \mathbb{Q}}^{*}$ os subespaços racionais gerados pelas raízes. Suponha que exista uma transformação linear invertivel sobre os racionais $\phi: \mathfrak{h}_{1 \mathbb{Q}}^{*} \rightarrow \mathfrak{h}_{2 \mathbb{Q}}^{*}$ tal que $\phi\left(\Pi_{1}\right)=\Pi_{2}$ (ou seja, seus diagramas de Dynkin coincidem).

Então, $\phi$ se estende a um isomorfismo $\tilde{\phi}: \mathfrak{g}^{1} \rightarrow \mathfrak{g}^{2}$.

Demonstração: A primeira afirmação que fazemos é: $\phi$ é uma isometria entre as formas de Cartan-Killing de $\mathfrak{h}_{1 \mathbb{Q}}^{*}$ e $\mathfrak{h}_{2 \mathbb{Q}}^{*}$. De fato, pelo lema 4.1.9 $\langle\phi(\alpha), \phi(\beta)\rangle=c\langle\alpha, \beta\rangle$, para algum escalar $c \neq 0$. Como 


$$
\begin{aligned}
\langle\alpha, \beta\rangle & =\sum_{\gamma \in \Pi_{1}} \gamma\left(H_{\alpha}\right) \gamma\left(H_{\beta}\right) \\
& =\sum_{\gamma \in \Pi_{1}}\langle\gamma, \alpha\rangle\langle\gamma, \beta\rangle \\
& =\sum_{\gamma \in \Pi_{1}} c\langle\phi(\gamma), \phi(\alpha)\rangle c\langle\phi(\gamma), \phi(\beta)\rangle \\
& =c^{2}\langle\phi(\alpha), \phi(\beta)\rangle
\end{aligned}
$$

temos $c=c^{2}$ e portanto $c=1$, concluindo que $\phi$ é uma isometria realmente.

Agora, para cada $\alpha \in \Pi_{1}$ tome $X_{\alpha} \in \mathfrak{g}^{1}$, tal que

$$
\left\langle X_{\alpha}, X_{-\alpha}\right\rangle=1
$$

Manteremos a notação $\left[X_{\alpha}, X_{\beta}\right]=m_{\alpha, \beta} X_{\alpha+\beta}$. O objetivo será encontrar uma base de $\mathfrak{g}^{2}$, formada por $Y_{\phi(\alpha)}$ tal que $\left.\left\langle Y_{\phi(\alpha)}, Y_{-\phi(\alpha)}\right\rangle=1 \mathrm{e}\left[Y_{\phi(\alpha)}\right), Y_{\phi(\beta)}\right]=m_{\alpha, \beta} Y_{\phi(\alpha)+\phi(\beta)}$. Assim $\tilde{\phi}\left(X_{\alpha}\right):=Y_{\phi(\alpha)}$ será um isomorfismo, devido a igualdade entre as constantes de estrutura das bases $\left\{X_{\alpha}\right\}$ e $\left\{Y_{\phi(\alpha)}\right\}$.

Seja $\Sigma \subset \Pi$ um sistema simples de raízes, e defina a ordem lexicográfica de $\mathfrak{h}_{\mathbb{Q}}^{*}$ por essa base. Faremos a construção do conjunto $\left\{Y_{\phi(\alpha)}\right\}$ por indução em relação a ordem em П. Para simplificar a notação, $\phi(\alpha)$ será denotado $\alpha^{\prime}\left(\phi(\alpha):=\alpha^{\prime}\right)$. Para as raízes $\alpha$ de altura um, como $\alpha^{\prime}$ é simples também, tome $Y_{\alpha^{\prime}} \in \mathfrak{g}_{\alpha^{\prime}}^{2}$. Tome também $Y_{-\alpha^{\prime}} \in \mathfrak{g}_{-\alpha^{\prime}}^{2}$ tal que

$$
\left\langle Y_{\alpha^{\prime}}, Y_{-\alpha^{\prime}}\right\rangle=1
$$

Seja

$$
\Pi_{n}=\{\alpha \in \Pi ; \alpha \text { é de altura } \mathrm{n} \text { ou }-\alpha \text { é de altura } \mathrm{n}\} .
$$

A hipótese de indução é que se $\alpha \in \Pi_{n-1}$, então $Y_{\alpha^{\prime}}$ está definido e se $\beta$, $\gamma$ e $\beta+\gamma$ $\in \Pi_{n-1}$, então

$$
\left[Y_{\beta^{\prime}}, Y_{\gamma^{\prime}}\right]=m_{\beta, \gamma} Y_{\beta^{\prime}+\gamma^{\prime}} .
$$

Provaremos essa afirmação para as raízes que pertencem ao conjunto $\Pi_{n}$. Seja $\delta$ uma raiz positiva de altura $n$. Podemos escrever $\delta=\alpha+\beta$, tal que $\alpha$ é simples e $\beta$ raiz positiva. Por hipótese de indução $Y_{\alpha^{\prime}}$ e $Y_{\beta^{\prime}}$ estão definidos. Defino $Y_{\gamma^{\prime}}$ pela equação

$$
\left[Y_{\alpha^{\prime}}, Y_{\beta^{\prime}}\right]=m_{\alpha, \beta} Y_{\alpha^{\prime}+\beta^{\prime}}=m_{\alpha, \beta} Y_{\gamma^{\prime}} .
$$

Para completar seja $Y_{-\delta^{\prime}}$ tal que

$$
\left\langle Y_{\delta^{\prime}}, Y_{-\delta^{\prime}}\right\rangle=1
$$

Uma vez definidos os elementos $Y_{\delta^{\prime}}$ para todo $\delta \in \Pi$, se tomarmos $\gamma, \delta$ e $\gamma+\delta \in \Pi_{n}$, pode-se escrever 


$$
\left[Y_{\gamma^{\prime}}, Y_{\delta^{\prime}}\right]=n_{\gamma, \delta} Y_{\gamma^{\prime}+\delta^{\prime}}
$$

Falta provar que $n_{\gamma, \delta}=m_{\gamma, \delta}$.

Vamos analisar os vários casos que podem ocorrer com $\gamma, \delta$ e $\gamma+\delta$.

1. Se $\gamma, \delta$ e $\gamma+\delta \in \Pi_{n-1}$. Então, por hipótese de indução, $n_{\gamma, \delta}=m_{\gamma, \delta}$.

2. Se $\gamma+\delta$ é uma raiz positiva de altura $n$. Nesse caso $\gamma$ e $\delta \in \Pi_{n-1}$. Agora escrevemos $\gamma+\delta=\alpha+\beta$, tal que $\alpha$ é raiz simples e $\beta$ raiz positiva de altura $n-1$, assim

$$
\left[Y_{\alpha^{\prime}}, Y_{\beta^{\prime}}\right]=m_{\alpha, \beta} Y_{\alpha^{\prime}+\beta^{\prime}}=m_{\alpha, \beta} Y_{\gamma^{\prime}+\delta^{\prime}} .
$$

Se $\{\gamma, \delta\}=\{\alpha, \beta\}$, então pelo definição das raízes de altura $n$, já temos $n_{\gamma, \delta}=m_{\alpha, \beta}=$ $m_{\gamma, \delta}$. Logo podemos supor que $\gamma$ e $\delta$ são diferentes de $\alpha$ e $\beta$. Como $(-\gamma-\delta+\alpha+\beta)=0$, podemos aplicar o lema 4.1 .8 às raízes $-\gamma,-\delta, \alpha$ e $\beta$, concluindo que

$$
m_{\alpha, \beta} m_{-\gamma,-\delta}=-m_{\beta,-\gamma} m_{\alpha,-\delta}-m_{-\gamma, \alpha} m_{\beta,-\delta} .
$$

Analogamente, aplica-se o lema 4.1.8 às raízes $-\gamma^{\prime},-\delta^{\prime}, \alpha^{\prime}$ e $\beta^{\prime}$, que nos dá

$$
n_{\alpha, \beta} n_{-\gamma,-\delta}=-n_{\beta,-\gamma} n_{\alpha,-\delta}-n_{-\gamma, \alpha} n_{\beta,-\delta} .
$$

Como $\alpha, \beta, \gamma$ e $\delta$ são raízes positivas em $\Pi_{n-1}$, temos $\beta+(-\gamma), \alpha+(-\delta),(-\gamma)+\alpha$ e $\beta+(-\delta) \in \Pi_{n-1}$. Portanto os segundos membros das equações 4.7 e 4.8 são iguais e portanto $m_{\alpha, \beta} m_{-\gamma,-\delta}=n_{\alpha, \beta} n_{-\gamma,-\delta}$. Mas pelo que foi feito na definição de $Y_{\gamma^{\prime}, \delta^{\prime}}$, já sabemos que $n_{\alpha, \beta}=m_{\alpha, \beta} \neq 0$, portanto

$$
n_{-\gamma,-\delta}=m_{-\gamma,-\delta} .
$$

Agora, mostraremos que $n_{-\alpha,-\beta}=m_{-\alpha,-\beta}$. Pelo lema 4.1 .6 aplicado a $\alpha$ e $\beta$ temos

$$
m_{\alpha, \beta} m_{-\alpha,-\beta}=-q(p+1) \frac{\langle\alpha, \alpha\rangle}{2},
$$

aplicando agora a $\alpha^{\prime}$ e $\beta^{\prime}$ temos

$$
n_{\alpha, \beta} n_{-\alpha,-\beta}=-q(p+1) \frac{\left\langle\alpha^{\prime}, \alpha^{\prime}\right\rangle}{2},
$$

mas $\langle\alpha, \alpha\rangle=\left\langle\alpha^{\prime}, \alpha^{\prime}\right\rangle$, portanto $m_{-\alpha,-\beta}=n_{-\alpha,-\beta}$. Para concluirmos iremos aplicar novamente o lema 4.1.8 às raízes $-\alpha,-\beta, \gamma$ e $\delta$. Fazendo a mesma análise temos

$$
m_{-\alpha,-\beta} m_{\gamma, \delta}=n_{-\alpha,-\beta} n_{\gamma, \delta}
$$

Finalmente concluímos que $m_{\gamma, \delta}=n_{\gamma, \delta}$. 
3. Se $\gamma+\delta$ é raiz negativa. Então, a raiz $-(\gamma+\delta)$ é positiva. Assim aplicando o caso anterior às raízes $-\gamma \mathrm{e}-\delta$ utilizando a mesma construção concluímos que $m_{\gamma, \delta}=n_{\gamma, \delta}$.

4. Se $\gamma$ ou $\delta$ é de altura $\pm n$. Suponha, por exemplo, que $-\gamma$ é de altura $n$. Logo $\delta$ é raiz positiva pois $\gamma+\delta \in \Pi_{n}$. Dessa forma as raízes $\delta$ e $-(\gamma+\delta)$, são duas raízes cuja a soma é uma raiz de altura $n$. O que nos permite aplicar a elas a resolução do segundo caso. Portanto $n_{\delta,-(\gamma+\delta)}=m_{\delta,-(\gamma+\delta)}$. Pelo lema 4.1.7 aplicado a $\gamma, \delta$ e $-(\gamma+\delta)$. Temos

$$
m_{\delta,-(\gamma+\delta)}=m_{\gamma, \delta}
$$

Analogamente, aplicando o lema 4.1 .7 a $\gamma^{\prime}, \delta^{\prime}$ e $-\left(\gamma^{\prime}+\delta^{\prime}\right)$, temos

$$
n_{\delta,-(\gamma+\delta)}=n_{\gamma, \delta}
$$

Portanto

$$
n_{\gamma, \delta}=m_{\gamma, \delta}
$$

Esses casos combrem todas as possibilidades, provando a hipótese de indução para as raízes de $\Pi_{n}$. Portanto, as constantes de estrutura das bases $\left\{X_{\alpha}\right\}$ e $\left\{Y_{\phi(\alpha)}\right\}$ são iguais e as álgebras $\mathfrak{g}^{1}$ e $\mathfrak{g}^{2}$ são isomorfas.

Esse teorema junto com a proposição 4.1.2 nos fornece o resultado mais forte dessa teoria: Os diagramas de Dynkin são um invariante completo. Dito de outra maneira: Duas álgebras de Lie semi-simples são isomorfas se e somente se os seus diagramas de Dynkin coincidem.

Para completar a discussão feita nesse capítulo, nos falta mostrar que cada um dos diagramas $A_{l}, B_{l}, C_{l}, D_{l}, E_{6}, E_{7}, E_{8}, F_{4}$ e $G_{2}$ são realmente diagramas associados a espaços de raízes de subálgebras de Cartan de álgebras semi-simples. Faremos essa realização para as álgebras clássicas.

\subsection{Realizações das àlgebras clássicas}

Iremos realizar os diagramas que são associados às chamadas álgebras clássicas, que são as associadas aos diagramas $A_{l}, B_{l}, C_{l}$ e $D_{l}$. Pelo teorema 4.1.4, ao mostrarmos que o diagrama dessas álgebras é conexo concluiremos que essas álgebras são simples.

Teorema 4.2.1. Seja $\mathfrak{g}$ a algebra de Lie das matrizes $(l+1) \times(l+1)$ que têm traço zero, $\operatorname{sl}(l+1)$. Então o diagrama associado a $\mathfrak{g}$ é $A_{l}$.

Demonstração: Pelo que foi feito na seção 1.7, sabemos que tomando h a subálgebra de Cartan das matrizes diagonais em $s l(l+1)$, então o sistema simples de raízes será $\Sigma=\left\{\lambda_{1}-\lambda_{2}, \ldots, \lambda_{l}-\lambda_{l+1}\right\}$, onde $\lambda_{i}$ são os funcionais $\lambda_{i}: \operatorname{diag}\left\{a_{1}, \ldots, a_{l+1}\right\} \longmapsto a_{i}$. As raízes positivas são

$$
\Pi_{+}=\left\{\lambda_{i}-\lambda_{j}, i<j\right\}
$$


Se utilizarmos a notação $\alpha_{i j}=\lambda_{i}-\lambda_{j}$, o número de Killing é

$$
\frac{2\left\langle\alpha_{i j}, \alpha_{r s}\right\rangle}{\left\langle\alpha_{i j}, \alpha_{i j}\right\rangle}=\delta_{i r}-\delta_{i s}-\delta_{j r}+\delta_{j s},
$$

portanto o diagrama desse sistema simples de raízes é $A_{l}$.

Teorema 4.2.2. Seja $\mathfrak{g}$ a álgebra de Lie das matrizes $(2 l+1) \times(2 l+1)$ em dimensão impar que são anti-simétricas em relação a identidade, so $(2 l+1)=\left\{A \in \operatorname{sl}(2 l+1), A^{t}+\right.$ $A=0\}$. Então o diagrama associado a $\mathfrak{g} e ́ B_{l}$.

Demonstração: Observe primeiramente que $\mathfrak{g}$ é a álgebra das matrizes de $s l(2 l+1)$ que são anti-simétricas em relação a forma quadrática não-degenerada, definida pela matriz identidade. Como o corpo de escalares é algebricamente fechado, duas formas quadráticas não-degeneradas são equivalentes. Logo as álgebras de matrizes antisimétricas em relação a formas quadráticas não-degeneradas distintas são isomorfas. De fato, sejam $J_{1}$ e $J_{2}$ matrizes simétricas que definem formas quadráticas equivalentes, ou seja, existe uma matriz inversível $g$ tal que $J_{1}=g^{t} J_{2} g$. Sejam

$$
\mathfrak{g}_{i}=\left\{A \in \operatorname{sl}(2 l+1): A^{t} J_{i}+J_{i} A=0\right\},
$$

para $i=1$, 2. Afirmação: $A \in \mathfrak{g}_{2}$ se e somente se $g^{-1} A g \in \mathfrak{g}_{1}$.

Prova da afirmação:

$\left(g^{-1} A g\right)^{t} J_{1}+J_{1}\left(g^{-1} A g\right)=g^{t} A^{t}\left(g^{-1}\right)^{t} J_{1}+\left(J_{1} g^{-1}\right) A g=g^{t} A^{t}\left(J_{2} g\right)+\left(g^{t} J_{2}\right) A g=g^{t}\left(A^{t} J_{2}+J_{2} A\right) g$.

Portanto como $g$ é invertível, temos $\left(g^{-1} A g\right)^{t} J_{1}+J_{1}\left(g^{-1} A g\right)=0$ se e somente se $A^{t} J_{2}+J_{2} A=0$. Concluindo que $g \mathfrak{g}_{2} g^{-1}=\mathfrak{g}_{1}$, isto é, as álgebras são isomorfas. Logo, $s o(2 l+1)$ é uma álgebra que pode ser realizada de várias maneiras, dependendo da escolha da forma quadrática.

A forma escolhida para a nossa realização é dada pela matriz $J$,

$$
J=\left(\begin{array}{ccc}
1 & 0 & 0 \\
0 & 0 & 1_{l} \\
0 & 1_{l} & 0
\end{array}\right)
$$

onde $1_{l}$ é a matriz identidade $l \times l$. Essa matriz é simétrica e equivalente a matriz identidade $(2 l+1) \times(2 l+1)$, pois se

$$
g=\frac{1}{\sqrt{2}}\left(\begin{array}{ccc}
\sqrt{2} & 0 & 0 \\
0 & i 1_{l} & 1_{l} \\
0 & 1_{l} & 1_{l}
\end{array}\right)
$$

então $g^{t} I g=g^{t} g=J$. Portanto $s o(2 l+1)$ é uma álgebra isomorfa a álgebra das matrizes anti-simétricas em relação a $J$. 
Escrevendo a matriz $A,(2 l+1) \times(2 l+1)$, com blocos do mesmo tamanho que os blocos de $J$ e usando a condição $A^{t} J+J A=0$. Calculamos que a matriz $A$ tem a forma

$$
A=\left(\begin{array}{ccc}
0 & \beta & \gamma \\
-\gamma^{t} & a & b \\
-\beta^{t} & c & -a^{t}
\end{array}\right)
$$

$\operatorname{com} \beta$ e $\gamma$ duas matrizes $1 \times l$ e as demais matrizes $l \times l, b$ e $c$ anti-simétricas e $a$ matriz qualquer. Uma subálgebra de Cartan $\mathfrak{h}$ é a das matrizes diagonais. As matrizes são da forma

$$
H=\left(\begin{array}{ccc}
0 & 0 & 0 \\
0 & \Lambda & 0 \\
0 & 0 & -\Lambda
\end{array}\right)
$$

com $\Lambda$ uma matriz diagonal $l \times l$ qualquer. Como no caso de $A_{l}$, defina em $\mathfrak{h}$ os funcinais $\lambda_{j}: \Lambda=\operatorname{diag}\left\{a_{1}, \ldots, a_{l}\right\} \longmapsto a_{j}$, para $i=1, \ldots, l$.

Então os autovalores de $H \in \mathfrak{h}$ são 0 e $\pm \lambda_{j}(H)$. Os possíveis valores que uma raiz assume em $H$ são: $\pm \lambda_{j}(H)$ e $\pm \lambda_{j}(H) \pm \lambda_{k}(H)$, para $j, k=1, \ldots, l, j \neq k$. Pois, se supormos que $\pm 2\left(\lambda_{j}\right)(H)$ é auto-valor de $a d(H)$, então as matrizes que estão no autoespaço

$$
\mathfrak{g}_{2 \lambda_{j}}=\left\{X \in \mathfrak{g}: \forall H, \exists n \geq 0,\left(\operatorname{ad}(H)-2 \lambda_{j}(H)\right)(X)=0\right\}
$$

tem as entradas das diagonais de $c$ ou de $b$ não nulas. Mas $c$ e $b$ (definidas em 4.9) são anti-simétricas (de diagonal nula). Logo as raízes dessa subálgebra de Cartan e seus espaços de raízes correspondentes são:

- $\lambda_{j}, j=1, \ldots, l$, com o espaço de raízes formado pelas matrizes

$$
A=\left(\begin{array}{ccc}
0 & 0 & \gamma \\
-\gamma^{t} & 0 & 0 \\
0 & 0 & 0
\end{array}\right)
$$

onde $\gamma=\left(0, \ldots, x_{j}, \ldots, 0\right), a=b=c=0$ e $\beta=0$.

- $-\lambda_{j}, j=1, \ldots, l$, com o espaço de raízes formado pelas matrizes

$$
A=\left(\begin{array}{ccc}
0 & \beta & 0 \\
0 & 0 & 0 \\
-\beta^{t} & 0 & 0
\end{array}\right)
$$

onde $\beta=\left(0, \ldots, x_{j}, \ldots, 0\right), a=b=c=0$ e $\gamma=0$.

- $\lambda_{i}-\lambda_{j}, i \neq j$, com o espaço de raízes formado pelas matrizes

$$
A=\left(\begin{array}{ccc}
0 & 0 & 0 \\
0 & a & 0 \\
0 & 0 & -a^{t}
\end{array}\right)
$$


onde $a$ é uma matriz $l \times l$ cuja única entrada não nula é $a_{i j}, b=c=0$ e $\gamma=\beta=0$. - $\lambda_{i}+\lambda_{j}, i \neq j$, com o espaço de raízes formado pelas matrizes

$$
A=\left(\begin{array}{lll}
0 & 0 & 0 \\
0 & 0 & b \\
0 & 0 & 0
\end{array}\right)
$$

onde $b$ é uma matriz $l \times l$ em que as únicas entradas não nulas são $b_{i j}$ e $b_{j i}, a=c=0$ e $\gamma=\beta=0$

- $-\left(\lambda_{i}+\lambda_{j}\right), i \neq j$, com o espaço de raízes formado pelas matrizes

$$
A=\left(\begin{array}{lll}
0 & 0 & 0 \\
0 & 0 & 0 \\
0 & c & 0
\end{array}\right)
$$

onde $c$ é uma matriz $l \times l$ em que as únicas entradas não nulas são $c_{i j}$ e $c_{j i}, a=c=0$ e $\gamma=\beta=0$.

Cada um desses espaços de raízes é de dimensão um e se para todo $\alpha \in \Pi, \alpha(H)=0$ então $H=0$. O conjunto gerador das raízes é

$$
\Sigma=\left\{\lambda_{1}-\lambda_{2}, \ldots, \lambda_{l-1}-\lambda_{l}, \lambda_{l}\right\}
$$

Esse conjunto $\Sigma$ é um sistema simples de raízes, pois tem o mesmo número de elementos que a dimensão de $\mathfrak{h}$ e toda raiz pode ser escrita como combinação de elementos de $\Sigma$ com coeficientes inteiros do mesmo sinal. De fato,

$$
\lambda_{j}=\left(\lambda_{j}-\lambda_{j+1}\right)+\ldots+\left(\lambda_{l-1}-\lambda_{l}\right)+\lambda_{l}
$$

Se $i<j$, temos

$$
\lambda_{i}+\lambda_{j}=\left(\lambda_{i}-\lambda_{i+1}\right)+\ldots+\left(\lambda_{j-1}-\lambda_{j}\right)+2\left(\lambda_{j}-\lambda_{j+1}\right)+2\left(\lambda_{j+1}-\lambda_{j+2}\right)+\ldots+2 \lambda_{l},
$$

e

$$
\lambda_{i}-\lambda_{j}=\left(\lambda_{i}-\lambda_{i+1}\right)+\ldots+\left(\lambda_{j-1}-\lambda_{j}\right),,
$$

que são as raízes positivas em $\Pi$.

Finalmente vamos mostrar a expressão da forma de Cartan-Killing restrita a $\mathfrak{h}$. Utilizaremos a mesma técnica, da fórmula de polarização, que relaciona uma forma quadrática com a forma bilinear associada. Seja $H \in \mathfrak{g}$ definido em 4.10, então:

$$
\begin{aligned}
\operatorname{tr}\left(a d(H)^{2}\right)=\langle H, H\rangle & =2 \sum_{i=1}^{l} a_{i}^{2}+\sum_{i \neq j}\left(a_{i}-a_{j}\right)^{2}+\sum_{i \neq j}\left(a_{i}+a_{j}\right)^{2}= \\
2 \sum_{i=1}^{l} a_{i}^{2}+4 \sum_{i<j}\left(a_{i}^{2}+a_{j}^{2}\right) & =2 \sum_{i=1}^{l} a_{i}^{2}+4(l-1) \sum_{i=1}^{l} a_{i}^{2}= \\
(2+4(l-1)) \sum_{i=1}^{l} a_{i}^{2} & =2(2 l-1) \sum_{i=1}^{l} a_{i}^{2} .
\end{aligned}
$$


Portanto, pela fórmula de polarização, para todo $H^{\prime} \in \mathfrak{h}$

$$
\left\langle H, H^{\prime}\right\rangle=2(2 l-1) \sum_{i=1}^{l} a_{i} a_{i}^{\prime},
$$

onde $\Lambda^{\prime}=\operatorname{diag}\left\{a_{1}^{\prime}, a_{2}^{\prime}, \ldots, a_{l}^{\prime}\right\}$ é a matriz diagonal que define $H^{\prime}$.

Vamos denotar agora, por $\Lambda_{\alpha}$, a matriz diagonal que define $H_{\alpha} \in \mathfrak{h}$. Então,

$$
\begin{gathered}
\Lambda_{\lambda_{i}}=\frac{1}{2(2 l-1)} \operatorname{diag}\left\{0, \ldots, 1_{i}, \ldots, 0\right\}, \\
\Lambda_{\lambda_{i}-\lambda_{j}}=\frac{1}{2(2 l-1)} \operatorname{diag}\left\{0, \ldots, 1_{i}, \ldots,-1_{j}, \ldots, 0\right\}, \\
\Lambda_{\lambda_{i}+\lambda_{j}}=\frac{1}{2(2 l-1)} \operatorname{diag}\left\{0, \ldots, 1_{i}, \ldots, 1_{j}, \ldots, 0\right\} .
\end{gathered}
$$

Com essas expressões, encontramos os números de Killing associados a raízes. Note que

$$
\left\langle\lambda_{i}-\lambda_{j}, \lambda_{i}-\lambda_{j}\right\rangle=\frac{2(2 l-1)}{2(2 l-1) 2(2 l-1)} 2=\frac{1}{2 l-1}
$$

$\mathrm{e}$

$$
\left\langle\lambda_{l}, \lambda_{l}\right\rangle=\frac{2(2 l-1)}{2(2 l-1) 2(2 l-1)} 1=\frac{1}{2(2 l-1)} .
$$

Portanto os números de Killing são

$$
\begin{gathered}
2 \frac{\left\langle\lambda_{i}-\lambda_{i+1}, \lambda_{i+1}-\lambda_{i+2}\right\rangle}{\left\langle\lambda_{i}-\lambda_{i+1}, \lambda_{i}-\lambda_{i+1}\right\rangle}=2\left(\frac{\frac{-1}{2(2 l-1)}}{\frac{1}{2 l-1}}\right)=-1, \text { para } i=1, \ldots, l-1, \\
2 \frac{\left\langle\lambda_{l-1}-\lambda_{l}, \lambda_{l}\right\rangle}{\left\langle\lambda_{l}, \lambda_{l}\right\rangle}=2\left(\frac{\frac{-1}{2(2 l-1)}}{\frac{1}{2(2 l-1)}}\right)=-2 .
\end{gathered}
$$

Portanto esse sistema de raízes tem o diagrama $B_{l}$.

Teorema 4.2.3. Seja $\mathfrak{g}$ a algebra de Lie das matrizes $2 l \times 2 l$ que são anti-simétricas em relação a $J, s p(l)=\left\{A \in \operatorname{sl}(2 l), A J+J A^{t}=0\right\}$, onde $J$ é uma matriz escrita em blocos $l \times l$ como

$$
J=\left(\begin{array}{cc}
0 & -1 \\
1 & 0
\end{array}\right) .
$$

Então o diagrama associado a $\mathfrak{g}$ é $C_{l}$. 
Demonstração: Essa álgebra é chamada álgebra simplética. Escrevendo uma matriz $A$ em blocos $l \times l, A \in \operatorname{sp}(l)$ se e somente se $A$ é da forma

$$
A=\left(\begin{array}{cc}
\alpha & \beta \\
\gamma & -\alpha^{t}
\end{array}\right)
$$

$\operatorname{com} \beta$ e $\gamma$ matrizes simétricas. Novamente é fácil notar que a subálgebra $\mathfrak{h}$ das matrizes diagonais em $s p(l)$ é de Cartan. Os elementos são da forma

$$
H=\left(\begin{array}{cc}
\Lambda & 0 \\
0 & -\Lambda
\end{array}\right)
$$

$\operatorname{com} \Lambda=\operatorname{diag}\left\{a_{1}, \ldots, a_{l}\right\}$ uma matriz diagonal $l \times l$. Da mesma forma que na demonstração anterior, se $\lambda_{i}$ é o funcional linear que toma a entrada $a_{i}$, então como um autovalor de $a d(H)$ é a diferença de autovalores de $H$, as raízes são diferenças $\pm \lambda_{i} \pm \lambda_{j}$. Nesse caso $\pm 2 \lambda_{i}$ é uma raíz pois $\beta$ e $\gamma$ são matrizes simétricas, podendo então ter entradas não nulas nas diagonais. Dessa forma as raízes e seus espaços correspondentes são:

- $\lambda_{i}-\lambda_{j}, i \neq j$, com o espaço de raízes formado pelas matrizes

$$
A=\left(\begin{array}{cc}
\alpha & 0 \\
0 & -\alpha^{t}
\end{array}\right)
$$

onde $\alpha$ é uma matriz $l \times l$ cuja única entrada não nula é $i, j$ e $\beta=\gamma=0$.

- $\lambda_{i}+\lambda_{j}, i, j=1, \ldots, l$, com o espaço de raízes formado pelas matrizes

$$
A=\left(\begin{array}{ll}
0 & \beta \\
0 & 0
\end{array}\right)
$$

onde $\beta$ é uma matriz $l \times l$ com entradas não nula apenas em $i, j$ e $j, i . \alpha=\gamma=0$. - $-\left(\lambda_{i}+\lambda_{j}\right), i, j=1, \ldots, l$, com o espaço de raízes formado pelas matrizes

$$
A=\left(\begin{array}{ll}
0 & 0 \\
\gamma & 0
\end{array}\right)
$$

onde $\gamma$ é uma matriz $l \times l$ com entradas não nula apenas em $i, j$ e $j, i . \alpha=\beta=0$. Um sistema simples de raízes é

$$
\Sigma=\left\{\lambda_{1}-\lambda_{2}, \ldots, \lambda_{l-1}-\lambda_{l}, 2 \lambda_{l}\right\} .
$$

As raízes positivas são escritas como combinações lineares de elementos de $\Sigma$ como

$$
\lambda_{i}-\lambda_{j}=\left(\lambda_{i}-\lambda_{i+1}\right)+\ldots+\left(\lambda_{j-1}-\lambda_{j}\right)
$$

se $i<j$ e

$$
\lambda_{i}+\lambda_{j}=\left(\lambda_{i}-\lambda_{i+1}\right)+\ldots+\left(\lambda_{j-1}-\lambda_{j}\right)+2\left(\lambda_{j}-\lambda_{j+1}\right)+\ldots+2\left(\lambda_{l-1}-\lambda_{l}\right)+2 \lambda_{l},
$$


se $i \leq j, i, j=1, \ldots, l$.

Iremos encontrar agora a forma de Cartan-Killing em $\mathfrak{h}$. Seja $H$ a matriz definida em 4.12 então,

$$
\begin{aligned}
\operatorname{tr}\left(a d(H)^{2}\right) & =\langle H, H\rangle=\sum_{i \neq j}\left(a_{i}-a_{j}\right)^{2}+\sum_{i \neq j}\left(a_{i}+a_{j}\right)^{2}+8 \sum_{i=1}^{l} a_{i}^{2}= \\
4 \sum_{i<j} a_{i}^{2}+a_{j}^{2}+8 \sum_{i=1}^{l} a_{i}^{2} & =4(l-1) \sum_{i=1}^{l} a_{i}^{2}+8 \sum_{i=1}^{l} a_{i}^{2}=4(l+1) \sum_{i=1}^{l} a_{i}^{2} .
\end{aligned}
$$

Novamente por polarização,

$$
\left\langle H, H^{\prime}\right\rangle=4(l+1) \sum_{i=1}^{l} a_{i} a_{i}^{\prime}
$$

se $\Lambda^{\prime}=\operatorname{diag}\left\{a_{1}^{\prime}, \ldots, a_{l}^{\prime}\right\}$ é a matriz que define $H^{\prime}$. Assim as matrizes $H_{\alpha}$, dadas por $\Lambda_{\alpha}$, tem a forma

$$
\begin{gathered}
\Lambda_{\lambda_{i}-\lambda_{j}}=\frac{1}{4(l+1)} \operatorname{diag}\left\{0, \ldots, 1_{i}, \ldots,-1_{j}, \ldots, 0\right\}, \\
\Lambda_{\lambda_{i}+\lambda_{j}}=\frac{1}{4(l+1)} \operatorname{diag}\left\{0, \ldots, 1_{i}, \ldots, 1_{j}, \ldots, 0\right\}, i \neq j, \\
\Lambda_{2 \lambda_{i}}=\frac{1}{2(l+1)} \operatorname{diag}\left\{0, \ldots, 1_{i}, \ldots, 0\right\} .
\end{gathered}
$$

Com essas expressões encontramos os números de Killing associados a raízes. Note que

$$
\left\langle\lambda_{i}-\lambda_{i+1}, \lambda_{i}-\lambda_{i+1}\right\rangle=4(l+1) \frac{2}{4(l+1) 4(l+1)}=\frac{1}{2(l+1)}
$$

$\mathrm{e}$

$$
\left\langle 2 \lambda_{l}, 2 \lambda_{l}\right\rangle=4(l+1) \frac{4}{4(l+1)^{2}}=\frac{1}{(l+1)} .
$$

Portanto os números de Killing são

$$
\begin{gathered}
2 \frac{\left\langle\lambda_{i}-\lambda_{i+1}, \lambda_{i+1}-\lambda_{i+2}\right\rangle}{\left\langle\lambda_{i}-\lambda_{i+1}, \lambda_{i}-\lambda_{i+1}\right\rangle}=2\left(\frac{\frac{-1}{4(l+1)}}{\frac{1}{2(l+1)}}\right)=-1, \text { para } i=1, \ldots, l-1, \\
2 \frac{\left\langle\lambda_{l-1}-\lambda_{l}, 2 \lambda_{l}\right\rangle}{\left\langle\lambda_{l-1}-\lambda_{l}, \lambda_{l-1}-\lambda_{l}\right\rangle}=-2 .
\end{gathered}
$$

Portanto o diagrama de $\mathfrak{g}$ é $C_{l}$. 
Teorema 4.2.4. Seja $\mathfrak{g}$ a álgebra de Lie das matrizes de dimensão par que são antisimétricas em relação a identidade, so $(2 l)=\left\{A \in \operatorname{sl}(2 l), A+A^{t}=0\right\}$. Então o diagrama associado a $\mathfrak{g}$ é $D_{l}$.

Demonstração: Como no caso do diagrama $B_{l}$ em que tínhamos essas matrizes, só que em dimensão ímpar, a álgebra $\mathfrak{g}$ é isomorfa à álgebra das matrizes anti-simétricas em relação a uma matriz identidade ou de uma matriz que seja equivalente a identidade. Tome $J$ a matriz escrita em blocos $l \times l$

$$
J=\left(\begin{array}{ll}
0 & 1 \\
1 & 0
\end{array}\right)
$$

Assim a álgebra $s o(2 l)$ é isomorfa a álgebra

$$
\left\{A \in \operatorname{sl}(2 l): A J+J A^{t}=0\right\} .
$$

Para facilitar denotaremos também por $s o(2 l)$. Assim $A \in s o(2 l)$ se e somente se

$$
A=\left(\begin{array}{cc}
\alpha & \beta \\
\gamma & -\alpha^{t}
\end{array}\right)
$$

$\operatorname{com} \beta$ e $\gamma$ matrizes $l \times l$ anti-simétricas. Novamente a subálgebra $\mathfrak{h}$ das matrizes diagonais é de Cartan. Seus elementos se escrevem como

$$
H=\left(\begin{array}{cc}
\Lambda & 0 \\
0 & -\Lambda
\end{array}\right)
$$

onde $\Lambda$ é uma matriz diagonal. As raízes de $H$ são diferenças de autovalores de $H$. Aqui também $2 \lambda_{i}$ não é raiz pois $\beta$ e $\gamma$ são matrizes anti-simétricas. Logo as raízes e seus espaços correspondetes são dados por:

- $\lambda_{i}-\lambda_{j}, i \neq j$, com o espaço de raízes formado pelas matrizes (definidas em 4.13)

$$
A=\left(\begin{array}{cc}
\alpha & 0 \\
0 & -\alpha^{t}
\end{array}\right)
$$

onde $\alpha$ é uma matriz $l \times l$ cuja única entrada não nula é $i, j$ e $\beta=\gamma=0$.

- $\lambda_{i}+\lambda_{j}, i \neq j$, com o espaço de raízes formado pelas matrizes

$$
A=\left(\begin{array}{ll}
0 & \beta \\
0 & 0
\end{array}\right)
$$

onde $\beta$ é uma matriz $l \times l$ com entradas não nula apenas em $i, j$ e $j, i . \alpha=\gamma=0$.

- $-\left(\lambda_{i}+\lambda_{j}\right), i, j=1, \ldots, l$, com o espaço de raízes formado pelas matrizes

$$
A=\left(\begin{array}{ll}
0 & 0 \\
\gamma & 0
\end{array}\right)
$$


onde $\gamma$ é uma matriz $l \times l$ com entradas não nula apenas em $i, j$ e $j, i . \alpha=\beta=0$. Um sistema simples de raízes é

$$
\Sigma=\left\{\lambda_{1}-\lambda_{2}, \ldots, \lambda_{l-1}-\lambda_{l}, \lambda_{l-1}+\lambda_{l}\right\} .
$$

As raízes positivas são escritas como combinações lineares de elementos de $\Sigma$ como

$$
\lambda_{i}-\lambda_{j}=\left(\lambda_{i}-\lambda_{i+1}\right)+\ldots+\left(\lambda_{j-1}-\lambda_{j}\right)
$$

se $i<j$ e

$$
\begin{aligned}
& \quad \lambda_{i}+\lambda_{j}=\left(\lambda_{i}-\lambda_{j}\right)+2\left(\lambda_{j}-\lambda_{l-1}\right)+2\left(\lambda_{l-1}\right)= \\
& {\left[\left(\lambda_{i}-\lambda_{i+1}\right)+\ldots+\left(\lambda_{j-1}-\lambda_{j}\right)\right]+2\left[\left(\lambda_{j}-\lambda_{j+1}\right)+\ldots+\left(\lambda_{l-2}-\lambda_{l-1}\right)\right]+\left[\left(\lambda_{l-1}-\lambda_{l}\right)+\left(\lambda_{l-1}+\lambda_{l}\right)\right],}
\end{aligned}
$$

se $i \neq j$.

A forma de Cartan-Killing para $H$ definido em 4.14 é dada por

$$
\langle H, H\rangle=\sum_{i \neq j}\left(a_{i}-a_{j}\right)^{2}+\sum_{i \neq j}\left(a_{i}+a_{j}\right)^{2},
$$

ou ainda

$$
\langle H, H\rangle=4(l-1) \sum_{i=1}^{l} a_{i}^{2} .
$$

Concluímos que

$$
\left\langle H, H^{\prime}\right\rangle=4(l-1) \sum_{i=1}^{l} a_{i} a_{i}^{\prime} .
$$

Dada uma raiz $\alpha$ o seu dual correspondente $H_{\alpha}$, dado por $\Lambda_{\alpha}$, é

$$
\begin{gathered}
\Lambda_{\lambda_{i}-\lambda_{j}}=\frac{1}{4(l-1)} \operatorname{diag}\left\{0, \ldots, 1_{i}, \ldots,-1_{j}, \ldots, 0\right\}, i \neq j, \\
\Lambda_{\lambda_{i}+\lambda_{j}}=\frac{1}{4(l-1)} \operatorname{diag}\left\{0, \ldots, 1_{i}, \ldots, 1_{j}, \ldots, 0\right\}, i \neq j .
\end{gathered}
$$

$\mathrm{O}$ vértice em que ocorre a bifurcação é dado pela raiz $\lambda_{l-2}-\lambda_{l-1}$, pois ele é ligado a três outros vértices, visto que

$$
\begin{aligned}
\left\langle\lambda_{l-2}-\lambda_{l-1}, \lambda_{l-3}-\lambda_{l-2}\right\rangle & =\frac{1}{2(l-1)}, \\
\left\langle\lambda_{l-2}-\lambda_{l-1}, \lambda_{l-1}-\lambda_{l}\right\rangle & =\frac{1}{2(l-1)}, \\
\left\langle\lambda_{l-2}-\lambda_{l-1}, \lambda_{l-1}+\lambda_{l}\right\rangle & =\frac{1}{(l-1)} .
\end{aligned}
$$


É fácil verificar que os números de Killing das raízes são todos iguais a um. Portanto o diagrama associado a $\mathfrak{g}$ é $D_{l}$. 


\section{Bibliografia}

[1] San Martin, L. A. B. [1999] Álgebras de Lie. Campinas, SP: Editora da Unicamp.

[2] Humphreys, J.E. [1971] Introduction to Lie algebras and representation theory. Second printing, revised. Graduate Texts in Mathematics, 9. New York: Springer-Verlag.

[3] Jacobson, N. [1979] Lie algebras. Republication of the 1962 original. New York: Dover Publications, Inc. 